\title{
HYBRID EXTRAGRADIENT METHOD WITH REGULARIZATION FOR TRIPLE HIERARCHICAL VARIATIONAL INEQUALITIES WITH GENERAL MIXED EQUILIBRIUM AND SPLIT FEASIBILITY CONSTRAINTS
}

\author{
LU-CHUAN CENG
}

\begin{abstract}
In this paper, we introduce a hybrid extragradient iterative algorithm with regularization for solving the triple hierarchical variational inequality problem (THVIP) (defined over the common fixed point set of finitely many nonexpansive mappings and a strictly pseudocontraction) with constraints of a general mixed equilibrium problem (GMEP), a split feasibility problem (SFP) and a general system of variational inequalities (GSVI). The iterative algorithm is based on Korpelevich's extragradient method, viscosity approximation method, Mann's iteration method, hybrid steepest descent method and gradient-projection method (GPM) with regularization. It is proven that, under very mild conditions, the sequences generated by the proposed algorithm converge strongly to a unique solution of the THVIP. We also give the applications of our results for solving some special cases of the THVIP. The results presented in this paper improve and extend some corresponding ones in the earlier and recent literature.
\end{abstract}

\section{Introduction}

Let $H$ be a real Hilbert space with inner product $\langle\cdot, \cdot\rangle$ and norm $\|\cdot\|, C$ be a nonempty closed convex subset of $H$ and $P_{C}$ be the metric projection of $H$ onto $C$. Let $S: C \rightarrow C$ be a self-mapping on $C$. We denote by $\operatorname{Fix}(S)$ the set of fixed points of $S$ and by $\mathbf{R}$ the set of all real numbers. A mapping $\mathscr{A}: C \rightarrow H$ is called $L$-Lipschitz continuous if there exists a constant $L \geq 0$ such that

$$
\|\mathscr{A} x-\mathscr{A} y\| \leq L\|x-y\|, \quad \forall x, y \in C .
$$

Received May 10, 2015, accepted May 18, 2015.

2010 Mathematics Subject Classification. 49J30, 47H09, 47J20, 49M05.

Key words and phrases. Hybrid extragradient method with regularization, triple hierarchical variational inequality problem, general mixed equilibrium, general system of variational inequalities, nonexpansive mapping, strict pseudocontraction.

This research was partially supported by the Program for Outstanding Academic Leaders in Shanghai City (15XD1503100), Innovation Program of Shanghai Municipal Education Commission (15ZZ068) and Ph.D. Program Foundation of Ministry of Education of China (20123127110002). 
In particular, if $L=1$ then $\mathscr{A}$ is called a nonexpansive mapping; if $L \in[0,1)$ then $\mathscr{A}$ is called a contraction. A mapping $T: C \rightarrow C$ is called $\xi$-strictly pseudocontractive if there exists a constant $\xi \in[0,1)$ such that

$$
\|T x-T y\|^{2} \leq\|x-y\|^{2}+\xi\|(I-T) x-(I-T) y\|^{2}, \quad \forall x, y \in C .
$$

In particular, if $\xi=0$, then $T$ is a nonexpansive mapping.

Let $\mathscr{A}: C \rightarrow H$ be a nonlinear mapping on $C$. The variational inequality problem (VIP) associated with the set $C$ and the mapping $A$ is stated as follows: find $x^{*} \in C$ such that

$$
\left\langle\mathscr{A} x^{*}, x-x^{*}\right\rangle \geq 0, \quad \forall x \in C .
$$

The solution set of VIP $(1.1)$ is denoted by $\operatorname{VI}(C, \mathscr{A})$.

The VIP (1.1) was first discussed by Lions [1] and now is well known; there are a lot of different approaches towards solving VIP (1.1) in finite-dimensional and infinite-dimensional spaces, and the research is intensively continued. In 1976, Korpelevich [12] proposed an iterative algorithm for solving VIP (1.1) in Euclidean space $\mathbf{R}^{n}$ :

$$
\left\{\begin{array}{l}
y_{n}=P_{C}\left(x_{n}-\tau \mathscr{A} x_{n}\right), \\
x_{n+1}=P_{C}\left(x_{n}-\tau \mathscr{A} y_{n}\right), \quad \forall n \geq 0,
\end{array}\right.
$$

with $\tau>0$ a given number, which is known as the extragradient method. The literature on the VIP is vast and Korpelevich's extragradient method has received great attention given by many authors, who improved it in various ways; see e.g., [10, 13, 17, 38, 42, 45, 46, 47, 48] and references therein, to name but a few.

In particular, if $C$ is the fixed point set $\operatorname{Fix}(T)$ of a nonexpansive mapping $T$ and $S$ is another nonexpansive mapping (not necessarily with fixed points), then VIP (1.1) becomes the following problem: find $x^{*} \in \operatorname{Fix}(T)$ such that

$$
\left\langle(I-S) x^{*}, x-x^{*}\right\rangle \geq 0, \quad \forall x \in \operatorname{Fix}(T) .
$$

It is called a hierarchical variational inequality problem (HVIP), also known as a hierarchical fixed point problem, and it was studied in [20,21]. It is clear that if $S$ has fixed points, then they are solutions of VIP (1.2). It is worth mentioning that many practical problems can be written in the form of a HVIP; see for example [11, 16, 34] and the references therein. Such a problem is also a bilevel problem, in which we find a solution of the first problem subject to the condition that its solution is also a fixed point of a mapping.

Moudafi and Mainge [21] introduced and considered the following iterative scheme

$$
x_{n+1}=\lambda_{n} V x_{n}+\left(1-\lambda_{n}\right)\left(\alpha_{n} S x_{n}+\left(1-\alpha_{n}\right) T x_{n}\right),
$$


where $V: C \rightarrow C$ is a contraction and $\left\{\alpha_{n}\right\},\left\{\lambda_{n}\right\} \subset(0,1)$. They also proved the strong convergence of the sequence $\left\{x_{n}\right\}$ to a solution of VIP (1.2).

Yao, Liu and Marino [28] introduced and analyzed the following two-step iterative scheme

$$
\left\{\begin{array}{l}
y_{n}=\beta_{n} S x_{n}+\left(1-\beta_{n}\right) x_{n}, \\
x_{n+1}=\alpha_{n} V x_{n}+\left(1-\alpha_{n}\right) T y_{n},
\end{array} \quad \forall n \geq 0 .\right.
$$

where $V: C \rightarrow C$ is a contraction and $\left\{\alpha_{n}\right\},\left\{\beta_{n}\right\} \subset(0,1)$.

In this paper, we consider the following general mixed equilibrium problem (GMEP) (see, also, [26, 27]) of finding $x^{*} \in C$ such that

$$
\boldsymbol{\Theta}\left(x^{*}, y\right)+h\left(x^{*}, y\right) \geq 0, \quad \forall y \in C,
$$

where $\boldsymbol{\Theta}, h: C \times C \rightarrow \mathbf{R}$ are two bi-functions. We denote the set of solutions of GMEP (1.3) by $\operatorname{GMEP}(\boldsymbol{\Theta}, h)$. The GMEP (1.3) is very general, for examples, it includes the following equilibrium problems as special cases:

As an example, in $[16,17,44]$ the authors considered and studied the generalized equilibrium problem (GEP) which is to find $x^{*} \in C$ such that

$$
\boldsymbol{\Theta}\left(x^{*}, y\right)+\left\langle\mathscr{A} x^{*}, y-x^{*}\right\rangle \geq 0, \quad \forall y \in C .
$$

The set of solutions of GEP is denoted by $\operatorname{GEP}(\boldsymbol{\Theta}, \mathscr{A})$.

In $[18,21,26]$, the authors considered and studied the mixed equilibrium problem (MEP) which is to find $x^{*} \in C$ such that

$$
\boldsymbol{\Theta}\left(x^{*}, y\right)+\varphi(y)-\varphi\left(x^{*}\right) \geq 0, \quad \forall y \in C .
$$

The set of solutions of MEP is denoted by $\operatorname{MEP}(\boldsymbol{\Theta}, \varphi)$.

In $[5,20,29]$, the authors considered and studied the equilibrium problem (EP) which is to find $x \in C$ such that

$$
\boldsymbol{\Theta}\left(x^{*}, y\right) \geq 0, \quad \forall y \in C .
$$

The set of solutions of EP is denoted by $\operatorname{EP}(\Theta)$. It is easy to see that if $C=\operatorname{Fix}(T)$ and $\Theta(x, y)=$ $\langle(I-S) x, y-x\rangle$, VIP (1.2) can be reformulated as the above EP. It is worth to mention that the EP is an unified model of several problems, namely, variational inequality problems, optimization problems, saddle point problems, complementarity problems, fixed point problems, Nash equilibrium problems, etc.

Throughout this paper, it is assumed as in [40] that $\boldsymbol{\Theta}: C \times C \rightarrow \mathbf{R}$ is a bifunction satisfying conditions $(\theta 1)-(\theta 3)$ and $h: C \times C \rightarrow \mathbf{R}$ is a bi-function with restrictions (h1) $-(\mathrm{h} 3)$, where 
$(\theta 1) \boldsymbol{\Theta}(x, x)=0$ for all $x \in C$;

$(\theta 2) \boldsymbol{\Theta}$ is monotone (i.e., $\boldsymbol{\Theta}(x, y)+\boldsymbol{\Theta}(y, x) \leq 0, \forall x, y \in C)$ and upper hemicontinuous in the first variable, i.e., for each $x, y, z \in C$,

$$
\limsup _{t \rightarrow 0^{+}} \boldsymbol{\Theta}(t z+(1-t) x, y) \leq \boldsymbol{\Theta}(x, y)
$$

( $\theta 3) \boldsymbol{\Theta}$ is lower semicontinuous and convex in the second variable;

(h1) $h(x, x)=0$ for all $x \in C$;

(h2) $h$ is monotone and weakly upper semicontinuous in the first variable;

(h3) $h$ is convex in the second variable.

For $r>0$ and $x \in H$, let $T_{r}: H \rightarrow 2^{C}$ be a mapping defined by

$$
T_{r} x=\left\{z \in C: \boldsymbol{\Theta}(z, y)+h(z, y)+\frac{1}{r}\langle y-z, z-x\rangle \geq 0, \forall y \in C\right\}
$$

called the resolvent of $\Theta$ and $h$.

In 2012, Marino, Muglia and Yao [41] introduced a multi-step iterative method that generalizes the two-step method studied in [28] from two nonexpansive mappings to a finite family of nonexpansive mappings, and proved that the sequence generated by this method converges strongly to a common fixed point of the mappings which is also a solution of the GMEP (1.3). The multi-step iterative method in [41] involves the Mann-type iterative method and the viscosity approximation method. On the other hand, by combining the viscosity approximation method (see [22, 23]), hybrid steepest-descent method (see [2, 25]) and projection method, Ceng, Ansari and Yao [8] proposed an iterative algorithm that generates a sequence via the explicit scheme and proved that this sequence converges strongly to a unique solution of the following problem.

Problem 1.1. Let $F: C \rightarrow H$ be $\kappa$-Lipschitzian and $\eta$-strongly monotone with positive constants $\kappa, \eta>0$ (i.e., $\|F x-F y\| \leq \kappa\|x-y\|$ and $\langle F x-F y, x-y\rangle \geq \eta\|x-y\|^{2}$ for all $x, y \in C$ ). Let $V: C \rightarrow H$ be a $\rho$-contraction with coefficient $\rho \in[0,1)$ and $S, T: C \rightarrow C$ be two nonexpansive mappings with $\operatorname{Fix}(T) \neq \varnothing$. Let $0<\mu<2 \eta / \kappa^{2}$ and $0<\gamma \leq \tau$, where $\tau=1-\sqrt{1-\mu\left(2 \eta-\mu \kappa^{2}\right)}$. Then the objective is to find $x^{*} \in \Xi$ such that

$$
\left\langle(\mu F-\gamma V) x^{*}, x-x^{*}\right\rangle \geq 0, \quad \forall x \in \Xi
$$

where $\Xi$ denotes the solution set of the following HVIP: find $z^{*} \in \operatorname{Fix}(T)$ such that

$$
\left\langle(\mu F-\gamma S) z^{*}, z-z^{*}\right\rangle \geq 0, \quad \forall z \in \operatorname{Fix}(T) .
$$


Since Problem 1.1 has a triple hierarchical structure in contrast with bilevel programming problems (see [20,30]), that is, a variational inequality problem with a variational inequality constraint over the fixed point set $\operatorname{Fix}(T)$, we also call it a triple hierarchical variational inequality problem (THVIP), which is a generalization of the triple hierarchical constrained optimization problem (THCOP) considered by Iiduka [35, 36].

Motivated the above facts, Latif, Ceng and Ansari [24] introduced and studied the following THVIP:

Problem 1.2. Let $F: C \rightarrow H$ be $\kappa$-Lipschitzian and $\eta$-strongly monotone with positive constants $\kappa, \eta>0$. Let $V: C \rightarrow H$ be a $\rho$-contraction with coefficient $\rho \in[0,1)$ and $S_{i}, S, T: C \rightarrow C$ be nonexpansive mappings for all $i \in\{1, \ldots, N\}$. Assume that $\boldsymbol{\Theta}, h: C \times C \rightarrow \mathbf{R}$ are two bifunctions satisfying the hypotheses $(\theta 1)-(\theta 3)$ and (h1) $-(\mathrm{h} 3)$. Let $0<\mu<2 \eta / \kappa^{2}$ and $0<\gamma \leq \tau$, where $\tau=1-\sqrt{1-\mu\left(2 \eta-\mu \kappa^{2}\right)}$. Then the objective is to find $x^{*} \in \Xi$ such that

$$
\left\langle(\mu F-\gamma V) x^{*}, x-x^{*}\right\rangle \geq 0, \quad \forall x \in \Xi
$$

where $\Xi$ denotes the solution set of the following HVIP: find $z^{*} \in \Omega$ such that

$$
\left\langle(\mu F-\gamma S) z^{*}, z-z^{*}\right\rangle \geq 0, \quad \forall z \in \Omega,
$$

where $\Omega=\operatorname{Fix}(T) \cap \cap_{i=1}^{N} \operatorname{Fix}\left(S_{i}\right) \cap \operatorname{GMEP}(\Theta, h) \neq \varnothing$.

The authors [24] proposed the following multi-step hybrid viscosity iterative algorithm

$$
\left\{\begin{array}{l}
\boldsymbol{\Theta}\left(u_{n}, y\right)+h\left(u_{n}, y\right)+\frac{1}{r_{n}}\left\langle y-u_{n}, u_{n}-x_{n}\right\rangle \geq 0, \quad \forall y \in C, \\
y_{n, 1}=\beta_{n, 1} S_{1} u_{n}+\left(1-\beta_{n, 1}\right) u_{n}, \\
y_{n, i}=\beta_{n, i} S_{i} u_{n}+\left(1-\beta_{n, i}\right) y_{n, i-1}, \quad i=2, \ldots, N, \\
\left.x_{n+1}=P_{C}\left[\lambda_{n} \gamma\left(\alpha_{n} V x_{n}+\left(1-\alpha_{n}\right) S x_{n}\right)+\left(I-\lambda_{n} \mu F\right) T y_{n, N}\right)\right],
\end{array} \quad \forall n \geq 1 .\right.
$$

where $\left\{\lambda_{n}\right\},\left\{\alpha_{n}\right\},\left\{\beta_{n, i}\right\}, i=1, \ldots, N$ be sequences in $(0,1)$ and $\left\{r_{n}\right\}$ be a sequence in $(0, \infty)$ with $\liminf _{n \rightarrow \infty} r_{n}>0$.

They proved that, under appropriate conditions, the sequence $\left\{x_{n}\right\}$ converges strongly to a unique solution of Problem 1.2. It is worth pointing out that Problem 1.1 is a special case of Problem 1.3 whenever $\boldsymbol{\Theta} \equiv h \equiv 0$ and $S_{i} \equiv I$ for all $i \in\{1, \ldots, N\}$.

Furthermore, let $C$ and $Q$ be nonempty closed convex subsets of infinite-dimensional real Hilbert spaces $H$ and $\mathscr{H}$, respectively. The split feasibility problem (SFP) is to find a point $x^{*}$ with the property:

$$
x^{*} \in C \text { and } A x^{*} \in Q \text {, }
$$


where $A \in B(H, \mathscr{H})$ and $B(H, \mathscr{H})$ denotes the family of all bounded linear operators from $H$ to $\mathscr{H}$. We denote by $\Gamma$ the solution set of the SFP.

In 1994, the SFP was first introduced by Censor and Elfving [4], in finite-dimensional Hilbert spaces, for modeling inverse problems which arise from phase retrievals and in medical image reconstruction. A number of image reconstruction problems can be formulated as the SFP; see, e.g., [6] and the references therein. Recently, it is found that the SFP can also be applied to study intensity-modulated radiation therapy (IMRT); see, e.g., [9, 30] and the references therein. In the recent past, a wide variety of iterative methods have been used in signal processing and image reconstruction and for solving the SFP; see, e.g., [3, 6, 7, 9, 11, 13, 30, 45,46 ] and the references therein. A seemingly more popular algorithm that solves the SFP is the $C Q$ algorithm of Byrne [6, 7] which is found to be a gradient-projection method (GPM) in convex minimization. However, it remains a challenge how to implement the $C Q$ algorithm in the case where the projections $P_{C}$ and/or $P_{Q}$ fail to have closed-form expressions, though theoretically we can prove the (weak) convergence of the algorithm.

In 2010, Xu [3] gave a continuation of the study on the CQ algorithm and its convergence. He applied Mann's algorithm to the SFP and proposed an averaged CQ algorithm which was proved to be weakly convergent to a solution of the SFP. He also established the strong convergence result, which shows that the minimum-norm solution can be obtained.

In addition, let $F_{1}, F_{2}: C \rightarrow H$ be two mappings. Consider the following general system of variational inequalities (GSVI) of finding $\left(x^{*}, y^{*}\right) \in C \times C$ such that

$$
\begin{cases}\left\langle v_{1} F_{1} y^{*}+x^{*}-y^{*}, x-x^{*}\right\rangle \geq 0, & \forall x \in C, \\ \left\langle v_{2} F_{2} x^{*}+y^{*}-x^{*}, x-y^{*}\right\rangle \geq 0, & \forall x \in C,\end{cases}
$$

where $v_{1}>0$ and $v_{2}>0$ are two constants. The solution set of GSVI (1.6) is denoted by $\operatorname{GSVI}\left(C, F_{1}, F_{2}\right)$.

In particular, if $F_{1}=F_{2}=\mathscr{A}$, then the GSVI (1.6) reduces to the new system of variational inequalities (NSVI), which was defined by Verma [39]. Further, if $x^{*}=y^{*}$ additionally, then the NSVI reduces to the classical VIP (1.1). In 2008, Ceng, Wang and Yao [9] transformed the GSVI (1.6) into the fixed point problem of the mapping $G=P_{C}\left(I-v_{1} F_{1}\right) P_{C}\left(I-v_{2} F_{2}\right)$, that is, $G x^{*}=x^{*}$, where $y^{*}=P_{C}\left(I-v_{2} F_{2}\right) x^{*}$. Throughout this paper, the fixed point set of the mapping $G$ is denoted by $\operatorname{GSVI}(G)$.

Next, we introduce and study the THVIP (defined over the common fixed point set of finitely many nonexpansive mappings and a strict pseudocontraction) with constraints of GMEP (1.3), SFP (1.5) and GSVI (1.6).

Problem 1.3. Assume that 
(i) $F: C \rightarrow H$ is a $\kappa$-Lipschitzian and $\eta$-strongly monotone with positive constants $\kappa, \eta>0$ such that $0<\gamma \leq \tau$ and $0<\mu<2 \eta / \kappa^{2}$ with $\tau:=1-\sqrt{1-\mu\left(2 \eta-\mu \kappa^{2}\right)}$;

(ii) $F_{j}: C \rightarrow H$ is $\zeta_{j}$-inverse strongly monotone and $G:=P_{C}\left(I-v_{1} F_{1}\right) P_{C}\left(I-v_{2} F_{2}\right)$ with $v_{j} \in$ $\left(0,2 \zeta_{j}\right)$ for $j=1,2$;

(iii) $\boldsymbol{\Theta}, h: C \times C \rightarrow \mathbf{R}$ are two bi-functions satisfying the hypotheses $(\theta 1)-(\theta 3)$ and (h1) $-(\mathrm{h} 3)$;

(iv) $T: C \rightarrow C$ is a $\xi$-strict pseudocontraction, $S, S_{i}: C \rightarrow C$ are nonexpansive mappings for each $i=1, \ldots, N$ and $V: C \rightarrow H$ is a $\rho$-contraction with coefficient $\rho \in[0,1)$;

(v) $\Omega:=\operatorname{Fix}(T) \cap \cap_{i=1}^{N} \operatorname{Fix}\left(S_{i}\right) \cap \operatorname{GMEP}(\Theta, h) \cap \operatorname{GSVI}(G) \cap \Gamma \neq \varnothing$.

Then the objective is to find $x^{*} \in \Xi$ such that

$$
\left\langle(\mu F-\gamma V) x^{*}, x-x^{*}\right\rangle \geq 0, \quad \forall x \in \Xi,
$$

where $\Xi$ denotes the solution set of the following HVIP: find $z^{*} \in \Omega$ such that

$$
\left\langle(\mu F-\gamma S) z^{*}, z-z^{*}\right\rangle \geq 0, \quad \forall z \in \Omega .
$$

In this paper, we introduce a hybrid extragradient iterative algorithm with regularization for solving Problem 1.3, i.e., the THVIP (defined over the common fixed point set $\cap_{i=1}^{N} \operatorname{Fix}\left(S_{i}\right) \cap$ $\operatorname{Fix}(T)$ of finitely many nonexpansive mappings $S_{i}: C \rightarrow C, i=1, \ldots, N$ and a strictly pseudocontractive mapping $T: C \rightarrow C$ ) with constraints of GMEP (1.3), SFP (1.5) and GSVI (1.6). The iterative algorithm is based on Korpelevich's extragradient method, viscosity approximation method (see [22, 23]), Mann's iteration method, hybrid steepest-descent method (see [2, 25]) and gradient-projection method (GPM) with regularization. It is proven that, under very mild conditions, the sequences generated by the proposed algorithm converge strongly to a unique solution of Problem 1.3. We also give the applications of our results for solving some special cases of Problem 1.3. It is worth pointing out that Problem 1.1 is a special case of Problem 1.3 whenever $T$ is nonexpansive, $\nabla f=0, F_{1}=F_{2}=0, \boldsymbol{\Theta}=h=0$ and $S_{i}=I$ for $i \in\{1, \ldots, N\}$. Moreover, Problem 1.2 is also a special case of Problem 1.3 whenever $T$ is nonexpansive, $\nabla f=0$ and $F_{1}=F_{2}=0$.

\section{Preliminaries}

Throughout this paper, we assume that $H$ is a real Hilbert space whose inner product and norm are denoted by $\langle\cdot, \cdot\rangle$ and $\|\cdot\|$, respectively. Let $C$ be a nonempty closed convex subset of $H$. We write $x_{n} \rightarrow x$ to indicate that the sequence $\left\{x_{n}\right\}$ converges weakly to $x$ and $x_{n} \rightarrow x$ to indicate that the sequence $\left\{x_{n}\right\}$ converges strongly to $x$. Moreover, we use $\omega_{w}\left(x_{n}\right)$ to denote the weak $\omega$-limit set of the sequence $\left\{x_{n}\right\}$ and $\omega_{s}\left(x_{n}\right)$ to denote the strong $\omega$-limit set of the sequence $\left\{x_{n}\right\}$, i.e.,

$$
\omega_{w}\left(x_{n}\right):=\left\{x \in H: x_{n_{i}} \rightarrow x \text { for some subsequence }\left\{x_{n_{i}}\right\} \text { of }\left\{x_{n}\right\},\right.
$$


and

$$
\omega_{s}\left(x_{n}\right):=\left\{x \in H: x_{n_{i}} \rightarrow x \text { for some subsequence }\left\{x_{n_{i}}\right\} \text { of }\left\{x_{n}\right\}\right\}
$$

The metric (or nearest point) projection from $H$ onto $C$ is the mapping $P_{C}: H \rightarrow C$ which assigns to each point $x \in H$ the unique point $P_{C} x \in C$ satisfying the property

$$
\left\|x-P_{C} x\right\|=\inf _{y \in C}\|x-y\|=: d(x, C) .
$$

The following properties of projections are useful and pertinent to our purpose.

Proposition 2.1. Given any $x \in H$ and $z \in C$. One has

(i) $z=P_{C} x \Leftrightarrow\langle x-z, y-z\rangle \leq 0, \forall y \in C$;

(ii) $z=P_{C} x \Leftrightarrow\|x-z\|^{2} \leq\|x-y\|^{2}-\|y-z\|^{2}, \forall y \in C$;

(iii) $\left\langle P_{C} x-P_{C} y, x-y\right\rangle \geq\left\|P_{C} x-P_{C} y\right\|^{2}, \forall y \in H$, which hence implies that $P_{C}$ is nonexpansive and monotone.

Definition 2.1. A mapping $T: H \rightarrow H$ is said to be

(a) nonexpansive if

$$
\|T x-T y\| \leq\|x-y\|, \quad \forall x, y \in H
$$

(b) firmly nonexpansive if $2 T-I$ is nonexpansive, or equivalently, if $T$ is 1 -inverse strongly monotone (1-ism),

$$
\langle x-y, T x-T y\rangle \geq\|T x-T y\|^{2}, \quad \forall x, y \in H ;
$$

alternatively, $T$ is firmly nonexpansive if and only if $T$ can be expressed as

$$
T=\frac{1}{2}(I+S)
$$

where $S: H \rightarrow H$ is nonexpansive; projections are firmly nonexpansive.

Definition 2.2. A mapping $\mathscr{A}: C \rightarrow H$ is said to be

(i) monotone if

$$
\langle\mathscr{A} x-\mathscr{A} y, x-y\rangle \geq 0, \quad \forall x, y \in C ;
$$

(ii) $\eta$-strongly monotone if there exists a constant $\eta>0$ such that

$$
\langle\mathscr{A} x-\mathscr{A} y, x-y\rangle \geq \eta\|x-y\|^{2}, \quad \forall x, y \in C ;
$$

(iii) $\alpha$-inverse-strongly monotone if there exists a constant $\alpha>0$ such that

$$
\langle\mathscr{A} x-\mathscr{A} y, x-y\rangle \geq \alpha\|\mathscr{A} x-\mathscr{A} y\|^{2}, \quad \forall x, y \in C .
$$


It can be easily seen that if $T$ is nonexpansive, then $I-T$ is monotone. It is also easy to see that the projection $P_{C}$ is 1 -ism. Inverse strongly monotone (also referred to as co-coercive) operators have been applied widely in solving practical problems in various fields.

On the other hand, it is obvious that if $\mathscr{A}: C \rightarrow H$ is $\alpha$-inverse-strongly monotone, then $A$ is monotone and $\frac{1}{\alpha}$-Lipschitz continuous. Moreover, we also have that, for all $u, v \in C$ and $\lambda>0$,

$$
\begin{aligned}
\|(I-\lambda \mathscr{A}) u-(I-\lambda \mathscr{A}) v\|^{2} & =\|(u-v)-\lambda(\mathscr{A} u-\mathscr{A} v)\|^{2} \\
& =\|u-v\|^{2}-2 \lambda\langle\mathscr{A} u-\mathscr{A} v, u-v\rangle+\lambda^{2}\|\mathscr{A} u-\mathscr{A} v\|^{2} \\
& \leq\|u-v\|^{2}+\lambda(\lambda-2 \alpha)\|\mathscr{A} u-\mathscr{A} v\|^{2}
\end{aligned}
$$

So, if $\lambda \leq 2 \alpha$, then $I-\lambda \mathscr{A}$ is a nonexpansive mapping from $C$ to $H$.

In 2008, Ceng, Wang and Yao [9] transformed problem (1.6) into a fixed point problem in the following way:

Proposition 2.2 (see [9]). For given $\bar{x}, \bar{y} \in C,(\bar{x}, \bar{y})$ is a solution of the GSVI (1.6) if and only if $\bar{x}$ is a fixed point of the mapping $G: C \rightarrow C$ defined by

$$
G x=P_{C}\left(I-v_{1} F_{1}\right) P_{C}\left(I-v_{2} F_{2}\right) x, \quad \forall x \in C,
$$

where $\bar{y}=P_{C}\left(I-v_{2} F_{2}\right) \bar{x}$.

In particular, if the mapping $F_{j}: C \rightarrow H$ is $\zeta_{j}$-inverse-strongly monotone for $j=1,2$, then the mapping $G$ is nonexpansive provided $v_{j} \in\left(0,2 \zeta_{j}\right]$ for $j=1,2$. We denote by $\Xi$ denote the fixed point set of the mapping $G$.

The following result is easy to prove.

Proposition 2.3 (see [45]). Given $x^{*} \in H$, the following statements are equivalent:

(i) $x^{*}$ solves the SFP;

(ii) $x^{*}$ solves the fixed point equation

$$
P_{C}(I-\lambda \nabla f) x^{*}=x^{*},
$$

where $\lambda>0, \nabla f=A^{*}\left(I-P_{Q}\right) A$ and $A^{*}$ is the adjoint of $A$;

(iii) $x^{*}$ solves the variational inequality problem (VIP) of finding $x^{*} \in C$ such that

$$
\left\langle\nabla f\left(x^{*}\right), x-x^{*}\right\rangle \geq 0, \quad \forall x \in C .
$$


It is clear from Proposition 2.1 that

$$
\Gamma=\operatorname{Fix}\left(P_{C}(I-\lambda \nabla f)\right)=\operatorname{VI}(C, \nabla f), \quad \forall \lambda>0 .
$$

Definition 2.3. A mapping $T: H \rightarrow H$ is said to be an averaged mapping if it can be written as the average of the identity $I$ and a nonexpansive mapping, that is,

$$
T \equiv(1-\alpha) I+\alpha S
$$

where $\alpha \in(0,1)$ and $S: H \rightarrow H$ is nonexpansive. More precisely, when the last equality holds, we say that $T$ is $\alpha$-averaged. Thus firmly nonexpansive mappings (in particular, projections) are $\frac{1}{2}$-averaged mappings.

Proposition 2.4 (see [32]). Let $T: H \rightarrow H$ be a given mapping.

(i) $T$ is nonexpansive if and only if the complement $I-T$ is $\frac{1}{2}$-ism.

(ii) If $T$ is $v$-ism, then for $\gamma>0, \gamma T$ is $\frac{v}{\gamma}$-ism.

(iii) $T$ is averaged if and only if the complement $I-T$ is $v$-ism for some $v>1 / 2$. Indeed, for $\alpha \in(0,1), T$ is $\alpha$-averaged if and only if $I-T$ is $\frac{1}{2 \alpha}$-ism.

Proposition 2.5 (see [32, 50]). Let $S, T, V: H \rightarrow H$ be given operators.

(i) If $T=(1-\alpha) S+\alpha V$ for some $\alpha \in(0,1)$ and if $S$ is averaged and $V$ is nonexpansive, then $T$ is averaged.

(ii) $T$ is firmly nonexpansive if and only if the complement $I-T$ is firmly nonexpansive.

(iii) If $T=(1-\alpha) S+\alpha V$ for some $\alpha \in(0,1)$ and if $S$ is firmly nonexpansive and $V$ is nonexpansive, then $T$ is averaged.

(iv) The composite of finitely many averaged mappings is averaged. That is, if each of the mappings $\left\{T_{i}\right\}_{i=1}^{N}$ is averaged, then so is the composite $T_{1} \cdots T_{N}$. In particular, if $T_{1}$ is $\alpha_{1}$-averaged and $T_{2}$ is $\alpha_{2}$-averaged, where $\alpha_{1}, \alpha_{2} \in(0,1)$, then the composite $T_{1} T_{2}$ is $\alpha$ averaged, where $\alpha=\alpha_{1}+\alpha_{2}-\alpha_{1} \alpha_{2}$.

(v) If the mappings $\left\{T_{i}\right\}_{i=1}^{N}$ are averaged and have a common fixed point, then

$$
\bigcap_{i=1}^{N} \operatorname{Fix}\left(T_{i}\right)=\operatorname{Fix}\left(T_{1} \cdots T_{N}\right) .
$$

The notation $\operatorname{Fix}(T)$ denotes the set of all fixed points of the mapping $T$, that is, $\operatorname{Fix}(T)=$ $\{x \in H: T x=x\}$.

We need some facts and tools in a real Hilbert space $H$ which are listed as lemmas below.

Lemma 2.1. Let $X$ be a real inner product space. Then there holds the following inequality

$$
\|x+y\|^{2} \leq\|x\|^{2}+2\langle y, x+y\rangle, \quad \forall x, y \in X .
$$


Lemma 2.2. Let $H$ be a real Hilbert space. Then the following hold:

(a) $\|x-y\|^{2}=\|x\|^{2}-\|y\|^{2}-2\langle x-y, y\rangle$ for all $x, y \in H$;

(b) $\|\lambda x+\mu y\|^{2}=\lambda\|x\|^{2}+\mu\|y\|^{2}-\lambda \mu\|x-y\|^{2}$ for all $x, y \in H$ and $\lambda, \mu \in[0,1]$ with $\lambda+\mu=1$;

(c) If $\left\{x_{n}\right\}$ is a sequence in $H$ such that $x_{n} \rightarrow x$, it follows that

$$
\limsup _{n \rightarrow \infty}\left\|x_{n}-y\right\|^{2}=\limsup _{n \rightarrow \infty}\left\|x_{n}-x\right\|^{2}+\|x-y\|^{2}, \quad \forall y \in H .
$$

It is clear that, in a real Hilbert space $H, T: C \rightarrow C$ is $\xi$-strictly pseudocontractive if and only if the following inequality holds:

$$
\langle T x-T y, x-y\rangle \leq\|x-y\|^{2}-\frac{1-\xi}{2}\|(I-T) x-(I-T) y\|^{2}, \quad \forall x, y \in C .
$$

This immediately implies that if $T$ is a $\xi$-strictly pseudocontractive mapping, then $I-T$ is $\frac{1-\xi}{2}$-inverse strongly monotone; for further detail, we refer to [37] and the references therein. It is well known that the class of strict pseudocontractions strictly includes the class of nonexpansive mappings and that the class of pseudocontractions strictly includes the class of strict pseudocontractions.

Lemma 2.3 (see Proposition 2.1 of [37]). Let $C$ be a nonempty closed convex subset of a real Hilbert space $H$ and $T: C \rightarrow C$ be a mapping.

(i) If $T$ is a $\xi$-strictly pseudocontractive mapping, then $T$ satisfies the Lipschitzian condition

$$
\|T x-T y\| \leq \frac{1+\xi}{1-\xi}\|x-y\|, \quad \forall x, y \in C .
$$

(ii) If $T$ is a $\xi$-strictly pseudocontractive mapping, then the mapping $I-T$ is semiclosed at 0 , that is, if $\left\{x_{n}\right\}$ is a sequence in $C$ such that $x_{n}-\tilde{x}$ and $(I-T) x_{n} \rightarrow 0$, then $(I-T) \tilde{x}=0$.

(iii) If $T$ is $\xi$-(quasi-)strict pseudocontraction, then the fixed-point set $\operatorname{Fix}(T)$ of $T$ is closed and convex so that the projection $P_{\mathrm{Fix}(T)}$ is well defined.

Lemma 2.4 (see [38]). Let $C$ be a nonempty closed convex subset of a real Hilbert space $H$. Let $T: C \rightarrow C$ be a $\xi$-strictly pseudocontractive mapping. Let $\gamma$ and $\delta$ be two nonnegative real numbers such that $(\gamma+\delta) \xi \leq \gamma$. Then

$$
\|\gamma(x-y)+\delta(T x-T y)\| \leq(\gamma+\delta)\|x-y\|, \quad \forall x, y \in C .
$$

Lemma 2.5 (see Demiclosedness principle [43]). Let $C$ be a nonempty closed convex subset of a real Hilbert space $H$. Let $S$ be a nonexpansive self-mapping on $C$ with $\operatorname{Fix}(S) \neq \varnothing$. Then $I-S$ is demiclosed. That is, whenever $\left\{x_{n}\right\}$ is a sequence in $C$ weakly converging to some $x \in C$ and the sequence $\left\{(I-S) x_{n}\right\}$ strongly converges to some $y$, it follows that $(I-S) x=y$. Here $I$ is the identity operator of $H$. 
Lemma 2.6. Let $\mathscr{A}: C \rightarrow H$ be a monotone mapping. In the context of the variational inequality problem the characterization of the projection (see Proposition 2.1 (i)) implies

$$
u \in \operatorname{VI}(C, \mathscr{A}) \Leftrightarrow u=P_{C}(u-\lambda \mathscr{A} u), \quad \forall \lambda>0 .
$$

Let $C$ be a nonempty closed convex subset of a real Hilbert space $H$. We introduce some notations. Let $\lambda$ be a number in $(0,1]$ and let $\mu>0$. Associating with a nonexpansive mapping $T: C \rightarrow C$, we define the mapping $T^{\lambda}: C \rightarrow H$ by

$$
T^{\lambda} x:=T x-\lambda \mu F(T x), \quad \forall x \in C,
$$

where $F: C \rightarrow H$ is an operator such that, for some positive constants $\kappa, \eta>0, F$ is $\kappa$-Lipschitzian and $\eta$-strongly monotone on $C$; that is, $F$ satisfies the conditions:

$$
\|F x-F y\| \leq \kappa\|x-y\| \quad \text { and } \quad\langle F x-F y, x-y\rangle \geq \eta\|x-y\|^{2}
$$

for all $x, y \in C$.

Lemma 2.7 (see Lemma 3.1 of [25]). $T^{\lambda}$ is a contraction provided $0<\mu<\frac{2 \eta}{\kappa^{2}}$; that is,

$$
\left\|T^{\lambda} x-T^{\lambda} y\right\| \leq(1-\lambda \tau)\|x-y\|, \quad \forall x, y \in C,
$$

where $\tau=1-\sqrt{1-\mu\left(2 \eta-\mu \kappa^{2}\right)} \in(0,1]$.

\section{Remark 2.1.}

(i) Since $F$ is $\kappa$-Lipschitzian and $\eta$-strongly monotone on $C$, we get $0<\eta \leq \kappa$. Hence, whenever $0<\mu<\frac{2 \eta}{\kappa^{2}}$, we have

$$
\begin{aligned}
0 & \leq(1-\mu \eta)^{2}=1-2 \mu \eta+\mu^{2} \eta^{2} \\
& \leq 1-2 \mu \eta+\mu^{2} \kappa^{2} \\
& <1-2 \mu \eta+\frac{2 \eta}{\kappa^{2}} \mu \kappa^{2}=1,
\end{aligned}
$$

which implies

$$
0<1-\sqrt{1-2 \mu \eta+\mu^{2} \kappa^{2}} \leq 1 .
$$

So, $\tau=1-\sqrt{1-\mu\left(2 \eta-\mu \kappa^{2}\right)} \in(0,1]$.

(ii) In Lemma 2.7, put $F=\frac{1}{2} I$ and $\mu=2$. Then we know that $\kappa=\eta=\frac{1}{2}, 0<\mu=2<\frac{2 \eta}{\kappa^{2}}=4$ and

$$
\tau=1-\sqrt{1-\mu\left(2 \eta-\mu \kappa^{2}\right)}=1-\sqrt{1-2\left(2 \times \frac{1}{2}-2 \times\left(\frac{1}{2}\right)^{2}\right)}=1 .
$$

Lemma 2.8 (see Lemma 2.1 of [49]). Let $\left\{a_{n}\right\}$ be a sequence of nonnegative real numbers satisfying

$$
a_{n+1} \leq\left(1-\beta_{n}\right) a_{n}+\beta_{n} \gamma_{n}+\delta_{n}, \quad \forall n \geq 0,
$$

where $\left\{\beta_{n}\right\},\left\{\gamma_{n}\right\}$ and $\left\{\delta_{n}\right\}$ satisfy the following conditions: 
(i) $\left\{\beta_{n}\right\} \subset[0,1]$ and $\sum_{n=0}^{\infty} \beta_{n}=\infty$;

(ii) either limsup $\operatorname{sum}_{n \rightarrow \infty} \gamma_{n} \leq 0$ or $\sum_{n=0}^{\infty} \beta_{n}\left|\gamma_{n}\right|<\infty$;

(iii) $\delta_{n} \geq 0$ for all $n \geq 0$, and $\sum_{n=1}^{\infty} \delta_{n}<\infty$.

Then, $\lim _{n \rightarrow \infty} a_{n}=0$.

In the sequel, we will indicate with $\operatorname{GMEP}(\boldsymbol{\Theta}, h)$ the solution set of GMEP (1.3).

Lemma 2.9 (see [40]). Let $C$ be a nonempty closed convex subset of a real Hilbert space $H$. Let $\boldsymbol{\Theta}: C \times C \rightarrow \mathbf{R}$ be a bifunction satisfying conditions $(\theta 1)-(\theta 3)$ and $h: C \times C \rightarrow \mathbf{R}$ is a bi-function with restrictions (h1)-(h3). Moreover, let us suppose that

(H) for fixed $r>0$ and $x \in C$, there exist a bounded $K \subset C$ and $\hat{x} \in K$ such that for all $z \in$ $C \backslash K,-\boldsymbol{\Theta}(\hat{x}, z)+h(z, \hat{x})+\frac{1}{r}\langle\hat{x}-z, z-x\rangle<0$.

For $r>0$ and $x \in H$, the mapping $T_{r}: H \rightarrow 2^{C}$ (i.e., the resolvent of $\Theta$ and $h$ ) has the following properties:

(i) $T_{r} x \neq \varnothing$;

(ii) $T_{r} x$ is a singleton;

(iii) $T_{r}$ is firmly nonexpansive;

(iv) $\operatorname{GMEP}(\Theta, h)=\operatorname{Fix}\left(T_{r}\right)$ and it is closed and convex.

Lemma 2.10 (see [40]). Let us suppose that $(\theta 1)-(\theta 3)$, (h1)-(h3) and $(\mathrm{H})$ hold. Let $x, y \in$ $H, r_{1}, r_{2}>0$. Then

$$
\left\|T_{r_{2}} y-T_{r_{1}} x\right\| \leq\|y-x\|+\left|\frac{r_{2}-r_{1}}{r_{2}}\right|\left\|T_{r_{2}} y-y\right\| .
$$

Lemma 2.11 (see [41]). Suppose that the hypotheses of Lemma 2.9 are satisfied. Let $\left\{r_{n}\right\}$ be a sequence in $(0, \infty)$ with $\liminf _{n \rightarrow \infty} r_{n}>0$. Suppose that $\left\{x_{n}\right\}$ is a bounded sequence. Then the following statements are equivalent and true:

(a) if $\left\|x_{n}-T_{r_{n}} x_{n}\right\| \rightarrow 0$ as $n \rightarrow \infty$, each weak cluster point of $\left\{x_{n}\right\}$ satisfies the problem

$$
\boldsymbol{\Theta}(x, y)+h(x, y) \geq 0, \quad \forall y \in C,
$$

i.e., $\omega_{w}\left(x_{n}\right) \subseteq \operatorname{GMEP}(\boldsymbol{\Theta}, h)$.

(b) the demiclosedness principle holds in the sense that, if $x_{n} \rightarrow x^{*}$ and $\left\|x_{n}-T_{r_{n}} x_{n}\right\| \rightarrow 0$ as $n \rightarrow \infty$, then $\left(I-T_{r_{k}}\right) x^{*}=0$ for all $k \geq 1$.

Recall that a set-valued mapping $T: D(T) \subset H \rightarrow 2^{H}$ is called monotone if for all $x, y \in$ $D(T), f \in T x$ and $g \in T y$ imply

$$
\langle f-g, x-y\rangle \geq 0 .
$$


A set-valued mapping $T$ is called maximal monotone if $T$ is monotone and $(I+\lambda T) D(T)=H$ for each $\lambda>0$, where $I$ is the identity mapping of $H$. We denote by $G(T)$ the graph of $T$. It is known that a monotone mapping $T$ is maximal if and only if, for $(x, f) \in H \times H,\langle f-g, x-y\rangle \geq 0$ for every $(y, g) \in G(T)$ implies $f \in T x$. Next we provide an example to illustrate the concept of maximal monotone mapping.

Let $\mathscr{A}: C \rightarrow H$ be a monotone, $k$-Lipschitz-continuous mapping and let $N_{C} v$ be the normal cone to $C$ at $v \in C$, i.e.,

$$
N_{C} v=\{u \in H:\langle\nu-p, u\rangle \geq 0, \forall p \in C\} .
$$

Define

$$
\widetilde{T} v= \begin{cases}\mathscr{A} v+N_{C} v, & \text { if } v \in C, \\ \varnothing, & \text { if } v \notin C .\end{cases}
$$

Then, it is known in [19] that $\widetilde{T}$ is maximal monotone and

$$
0 \in \widetilde{T} v \Leftrightarrow v \in \operatorname{VI}(C, \mathscr{A}) .
$$

\section{Main Results}

Throughout this paper, assume that the SFP is consistent, that is, the solution set $\Gamma$ of the SFP is nonempty. Let $f: H \rightarrow \mathbf{R}$ be a continuous differentiable function. The minimization problem

$$
\min _{x \in C} f(x):=\frac{1}{2}\left\|A x-P_{Q} A x\right\|^{2}
$$

is ill-posed. Therefore, $\mathrm{Xu}$ [3] considered the following Tikhonov regularization problem:

$$
\min _{x \in C} f_{\alpha}(x):=\frac{1}{2}\left\|A x-P_{Q} A x\right\|^{2}+\frac{1}{2} \alpha\|x\|^{2},
$$

where $\alpha>0$ is the regularization parameter.

For arbitrarily given $x_{0} \in C$, we now propose the following hybrid extragradient iterative scheme with regularization:

$$
\left\{\begin{array}{l}
\boldsymbol{\Theta}\left(u_{n}, y\right)+h\left(u_{n}, y\right)+\frac{1}{r_{n}}\left\langle y-u_{n}, u_{n}-x_{n}\right\rangle \geq 0, \quad \forall y \in C, \\
y_{n, 1}=\beta_{n, 1} S_{1} u_{n}+\left(1-\beta_{n, 1}\right) u_{n}, \\
y_{n, i}=\beta_{n, i} S_{i} u_{n}+\left(1-\beta_{n, i}\right) y_{n, i-1}, \quad i=2, \ldots, N, \\
\tilde{y}_{n, N}=P_{C}\left(y_{n, N}-\lambda_{n} \nabla f_{\alpha_{n}}\left(y_{n, N}\right)\right), \\
y_{n}=\beta_{n} x_{n}+\gamma_{n} G P_{C}\left(y_{n, N}-\lambda_{n} \nabla f_{\alpha_{n}}\left(\tilde{y}_{n, N}\right)\right)+\sigma_{n} T G P_{C}\left(y_{n, N}-\lambda_{n} \nabla f_{\alpha_{n}}\left(\tilde{y}_{n, N}\right)\right), \\
x_{n+1}=P_{C}\left[\epsilon_{n} \gamma\left(\delta_{n} V x_{n}+\left(1-\delta_{n}\right) S x_{n}\right)+\left(I-\epsilon_{n} \mu F\right) y_{n}\right], \quad \forall n \geq 0,
\end{array}\right.
$$

where 
$F: C \rightarrow H$ is a $\kappa$-Lipschitzian and $\eta$-strongly monotone operator with positive constants $\kappa, \eta>0$ and $V: C \rightarrow H$ is a $\rho$-contraction with coefficient $\rho \in[0,1)$;

$F_{j}: C \rightarrow H$ is $\zeta_{j}$-inverse strongly monotone and $G:=P_{C}\left(I-v_{1} F_{1}\right) P_{C}\left(I-v_{2} F_{2}\right)$ with $v_{j} \in$ $\left(0,2 \zeta_{j}\right)$ for $j=1,2$;

$T: C \rightarrow C$ is a $\xi$-strict pseudocontraction and $S, S_{i}: C \rightarrow C$ are nonexpansive mappings for each $i=1, \ldots, N$;

$\boldsymbol{\Theta}, h: C \times C \rightarrow \mathbf{R}$ are two bi-functions satisfying the hypotheses of Lemma 2.9;

$0<\mu<2 \eta / \kappa^{2}$ and $0<\gamma \leq \tau$ with $\tau:=1-\sqrt{1-\mu\left(2 \eta-\mu \kappa^{2}\right)}$;

$\left\{\alpha_{n}\right\}$ is a sequence in $(0, \infty)$ with $\sum_{n=0}^{\infty} \alpha_{n}<\infty$;

$\left\{\lambda_{n}\right\}$ is a sequence in $\left(0, \frac{1}{\|A\|^{2}}\right)$ with $0<\liminf _{n \rightarrow \infty} \lambda_{n} \leq \limsup _{n \rightarrow \infty} \lambda_{n}<\frac{1}{\|A\|^{2}}$;

$\left\{\epsilon_{n}\right\},\left\{\beta_{n}\right\}$ are sequences in $(0,1)$ with $0<\liminf _{n \rightarrow \infty} \beta_{n} \leq \limsup _{n \rightarrow \infty} \beta_{n}<1$;

$\left\{\beta_{n, i}\right\}_{i=1}^{N},\left\{\delta_{n}\right\}$ are sequences in $(0,1)$;

$\left\{\gamma_{n}\right\},\left\{\sigma_{n}\right\}$ are sequences in $[0,1]$ with $\beta_{n}+\gamma_{n}+\sigma_{n}=1$ and $\left(\gamma_{n}+\sigma_{n}\right) \xi \leq \gamma_{n}, \forall n \geq 0$;

$\left\{r_{n}\right\}$ is a sequence in $(0, \infty)$ with $\liminf _{n \rightarrow \infty} r_{n}>0$ and $\liminf _{n \rightarrow \infty} \sigma_{n}>0$.

$\left\{S x_{n}\right\}$ is bounded, $\lim _{n \rightarrow \infty} \epsilon_{n} / \delta_{n}^{2}=0$ and $\max _{1 \leq i \leq N}\left\|x-S_{i} x\right\| \geq \bar{k} \cdot d(x, \Omega), \forall x \in C$ for some $\bar{k}>0$.

We start our main result from the following series of propositions.

Proposition 3.6. Let us suppose that $\Omega=\operatorname{Fix}(T) \cap \cap_{i=1}^{N} \operatorname{Fix}\left(S_{i}\right) \cap \operatorname{GMEP}(\Theta, h) \cap \operatorname{GSVI}(G) \cap \Gamma \neq \varnothing$. Then the sequences $\left\{x_{n}\right\},\left\{y_{n}\right\},\left\{y_{n, i}\right\}$ for all $i,\left\{u_{n}\right\}$ are bounded.

Proof. Since $0<\liminf _{n \rightarrow \infty} \lambda_{n} \leq \limsup _{n \rightarrow \infty} \lambda_{n}<\frac{1}{\|A\|^{2}}$ and $0<\liminf _{n \rightarrow \infty} \beta_{n} \leq \limsup _{n \rightarrow \infty}$ $\beta_{n}<1$, we may assume, without loss of generality, that $\left\{\lambda_{n}\right\} \subset[a, b] \subset\left(0, \frac{1}{\|A\|^{2}}\right)$ and $\left\{\beta_{n}\right\} \subset$ $[c, d] \subset(0,1)$. Now, let us show that $P_{C}\left(I-\lambda \nabla f_{\alpha}\right)$ is $\sigma$-averaged for each $\lambda \in\left(0, \frac{2}{\alpha+\|A\|^{2}}\right)$, where

$$
\sigma=\frac{2+\lambda\left(\alpha+\|A\|^{2}\right)}{4} \in(0,1)
$$

Indeed, it is easy to see that $\nabla f=A^{*}\left(I-P_{Q}\right) A$ is $\frac{1}{\|A\|^{2}}$-ism, that is,

$$
\langle\nabla f(x)-\nabla f(y), x-y\rangle \geq \frac{1}{\|A\|^{2}}\|\nabla f(x)-\nabla f(y)\|^{2} .
$$

Observe that

$$
\left(\alpha+\|A\|^{2}\right)\left\langle\nabla f_{\alpha}(x)-\nabla f_{\alpha}(y), x-y\right\rangle
$$




$$
\begin{aligned}
= & \left(\alpha+\|A\|^{2}\right)\left[\alpha\|x-y\|^{2}+\langle\nabla f(x)-\nabla f(y), x-y\rangle\right] \\
= & \alpha^{2}\|x-y\|^{2}+\alpha\langle\nabla f(x)-\nabla f(y), x-y\rangle+\alpha\|A\|^{2}\|x-y\|^{2} \\
& +\|A\|^{2}\langle\nabla f(x)-\nabla f(y), x-y\rangle \\
\geq & \alpha^{2}\|x-y\|^{2}+2 \alpha\langle\nabla f(x)-\nabla f(y), x-y\rangle+\|\nabla f(x)-\nabla f(y)\|^{2} \\
= & \|\alpha(x-y)+\nabla f(x)-\nabla f(y)\|^{2} \\
= & \left\|\nabla f_{\alpha}(x)-\nabla f_{\alpha}(y)\right\|^{2} .
\end{aligned}
$$

Hence, it follows that $\nabla f_{\alpha}=\alpha I+A^{*}\left(I-P_{Q}\right) A$ is $\frac{1}{\alpha+\|A\|^{2}}$-ism. Thus, $\lambda \nabla f_{\alpha}$ is $\frac{1}{\lambda\left(\alpha+\|A\|^{2}\right)}$-ism according to Proposition 2.4 (ii). By Proposition 2.4 (iii), the complement $I-\lambda \nabla f_{\alpha}$ is $\frac{\lambda\left(\alpha+\|A\|^{2}\right)}{2}$ averaged. Therefore, noting that $P_{C}$ is $\frac{1}{2}$-averaged and utilizing Proposition 2.5 (iv), we known that for each $\lambda \in\left(0, \frac{2}{\alpha+\|A\|^{2}}\right), P_{C}\left(I-\lambda \nabla f_{\alpha}\right)$ is $\sigma$-averaged with

$$
\sigma=\frac{1}{2}+\frac{\lambda\left(\alpha+\|A\|^{2}\right)}{2}-\frac{1}{2} \cdot \frac{\lambda\left(\alpha+\|A\|^{2}\right)}{2}=\frac{2+\lambda\left(\alpha+\|A\|^{2}\right)}{4} \in(0,1)
$$

This shows that $P_{C}\left(I-\lambda \nabla f_{\alpha}\right)$ is nonexpansive. Furthermore, for $\left\{\lambda_{n}\right\} \subset[a, b] \subset\left(0, \frac{1}{\|A\|^{2}}\right)$, we have

$$
a \leq \inf _{n \geq 0} \lambda_{n} \leq \sup _{n \geq 0} \lambda_{n} \leq b<\frac{1}{\|A\|^{2}}=\lim _{n \rightarrow \infty} \frac{1}{\alpha_{n}+\|A\|^{2}} .
$$

Without loss of generality, we may assume that

$$
a \leq \inf _{n \geq 0} \lambda_{n} \leq \sup _{n \geq 0} \lambda_{n} \leq b<\frac{1}{\alpha_{n}+\|A\|^{2}}, \quad \forall n \geq 0 .
$$

Consequently, it follows that for each integer $n \geq 0, P_{C}\left(I-\lambda_{n} \nabla f_{\alpha_{n}}\right)$ is $\sigma_{n}$-averaged with

$$
\sigma_{n}=\frac{1}{2}+\frac{\lambda_{n}\left(\alpha_{n}+\|A\|^{2}\right)}{2}-\frac{1}{2} \cdot \frac{\lambda_{n}\left(\alpha_{n}+\|A\|^{2}\right)}{2}=\frac{2+\lambda_{n}\left(\alpha_{n}+\|A\|^{2}\right)}{4} \in(0,1) .
$$

This immediately implies that $P_{C}\left(I-\lambda_{n} \nabla f_{\alpha_{n}}\right)$ is nonexpansive for all $n \geq 0$.

For simplicity, we write $t_{n}=P_{C}\left(y_{n, N}-\lambda_{n} \nabla f_{\alpha_{n}}\left(\tilde{y}_{n, N}\right)\right)$ and

$$
v_{n}=\epsilon_{n} \gamma\left(\delta_{n} V x_{n}+\left(1-\delta_{n}\right) S x_{n}\right)+\left(I-\epsilon_{n} \mu F\right) G y_{n}
$$

for all $n \geq 0$. Then $x_{n+1}=P_{C} v_{n}$ and $y_{n}=\beta_{n} x_{n}+\gamma_{n} t_{n}+\sigma_{n} T t_{n}$.

First of all, take a fixed $p \in \Omega$ arbitrarily. We observe that

$$
\left\|y_{n, 1}-p\right\| \leq\left\|u_{n}-p\right\| \leq\left\|x_{n}-p\right\|
$$

For all from $i=2$ to $i=N$, by induction, one proves that

$$
\left\|y_{n, i}-p\right\| \leq \beta_{n, i}\left\|u_{n}-p\right\|+\left(1-\beta_{n, i}\right)\left\|y_{n, i-1}-p\right\| \leq\left\|u_{n}-p\right\| \leq\left\|x_{n}-p\right\| .
$$


Thus we obtain that for every $i=1, \ldots, N$,

$$
\left\|y_{n, i}-p\right\| \leq\left\|u_{n}-p\right\| \leq\left\|x_{n}-p\right\| \text {. }
$$

For simplicity, we write $\tilde{p}=P_{C}\left(p-v_{2} F_{2} p\right), \tilde{t}_{n}=P_{C}\left(t_{n}-v_{2} F_{2} t_{n}\right)$ and $z_{n}=P_{C}\left(\tilde{y}_{n}-v_{1} F_{1} \tilde{y}_{n}\right)$ for each $n \geq 0$. Then $z_{n}=G y_{n}$ and

$$
p=P_{C}\left(I-v_{1} F_{1}\right) \tilde{p}=P_{C}\left(I-v_{1} F_{1}\right) P_{C}\left(I-v_{2} F_{2}\right) p=G p .
$$

Since $F_{j}: C \rightarrow H$ is $\zeta_{j}$-inverse strongly monotone and $0<v_{j}<2 \zeta_{j}$ for each $j=1,2$, we know that for all $n \geq 0$,

$$
\begin{aligned}
\left\|z_{n}-p\right\|^{2}= & \left\|G t_{n}-p\right\|^{2} \\
= & \left\|P_{C}\left(I-v_{1} F_{1}\right) P_{C}\left(I-v_{2} F_{2}\right) t_{n}-P_{C}\left(I-v_{1} F_{1}\right) P_{C}\left(I-v_{2} F_{2}\right) p\right\|^{2} \\
\leq & \left\|\left(I-v_{1} F_{1}\right) P_{C}\left(I-v_{2} F_{2}\right) t_{n}-\left(I-v_{1} F_{1}\right) P_{C}\left(I-v_{2} F_{2}\right) p\right\|^{2} \\
= & \left\|\left[P_{C}\left(I-v_{2} F_{2}\right) t_{n}-P_{C}\left(I-v_{2} F_{2}\right) p\right]-v_{1}\left[F_{1} P_{C}\left(I-v_{2} F_{2}\right) t_{n}-F_{1} P_{C}\left(I-v_{2} F_{2}\right) p\right]\right\|^{2} \\
\leq & \left\|P_{C}\left(I-v_{2} F_{2}\right) t_{n}-P_{C}\left(I-v_{2} F_{2}\right) p\right\|^{2} \\
& +v_{1}\left(v_{1}-2 \zeta_{1}\right)\left\|F_{1} P_{C}\left(I-v_{2} F_{2}\right) t_{n}-F_{1} P_{C}\left(I-v_{2} F_{2}\right) p\right\|^{2} \\
\leq & \left\|\left(I-v_{2} F_{2}\right) t_{n}-\left(I-v_{2} F_{2}\right) p\right\|^{2}+v_{1}\left(v_{1}-2 \zeta_{1}\right)\left\|F_{1} \tilde{t}_{n}-F_{1} \tilde{p}\right\|^{2} \\
= & \left\|\left(t_{n}-p\right)-v_{2}\left(F_{2} t_{n}-F_{2} p\right)\right\|^{2}+v_{1}\left(v_{1}-2 \zeta_{1}\right)\left\|F_{1} \tilde{t}_{n}-F_{1} \tilde{p}\right\|^{2} \\
\leq & \left\|t_{n}-p\right\|^{2}+v_{2}\left(v_{2}-2 \zeta_{2}\right)\left\|F_{2} t_{n}-F_{2} p\right\|^{2}+v_{1}\left(v_{1}-2 \zeta_{1}\right)\left\|F_{1} \tilde{t}_{n}-F_{1} \tilde{p}\right\|^{2} \\
\leq & \left\|t_{n}-p\right\|^{2} .
\end{aligned}
$$

From (3.1), (3.9) and the nonexpansivity of $P_{C}\left(I-\lambda_{n} \nabla f_{\alpha_{n}}\right)$, it follows that

$$
\begin{aligned}
\left\|\tilde{y}_{n, N}-p\right\|= & \left\|P_{C}\left(I-\lambda_{n} \nabla f_{\alpha_{n}}\right) y_{n, N}-P_{C}\left(I-\lambda_{n} \nabla f\right) p\right\| \\
\leq & \left\|P_{C}\left(I-\lambda_{n} \nabla f_{\alpha_{n}}\right) y_{n, N}-P_{C}\left(I-\lambda_{n} \nabla f_{\alpha_{n}}\right) p\right\| \\
& +\left\|P_{C}\left(I-\lambda_{n} \nabla f_{\alpha_{n}}\right) p-P_{C}\left(I-\lambda_{n} \nabla f\right) p\right\| \\
\leq & \left\|y_{n, N}-p\right\|+\left\|\left(I-\lambda_{n} \nabla f_{\alpha_{n}}\right) p-\left(I-\lambda_{n} \nabla f\right) p\right\| \\
\leq & \left\|x_{n}-p\right\|+\lambda_{n} \alpha_{n}\|p\| .
\end{aligned}
$$

Utilizing Lemma 2.1, we also have

$$
\begin{aligned}
\left\|\tilde{y}_{n, N}-p\right\|^{2}= & \left\|P_{C}\left(I-\lambda_{n} \nabla f_{\alpha_{n}}\right) y_{n, N}-P_{C}\left(I-\lambda_{n} \nabla f\right) p\right\|^{2} \\
= & \| P_{C}\left(I-\lambda_{n} \nabla f_{\alpha_{n}}\right) y_{n, N}-P_{C}\left(I-\lambda_{n} \nabla f_{\alpha_{n}}\right) p \\
& +P_{C}\left(I-\lambda_{n} \nabla f_{\alpha_{n}}\right) p-P_{C}\left(I-\lambda_{n} \nabla f\right) p \|^{2} \\
\leq & \left\|P_{C}\left(I-\lambda_{n} \nabla f_{\alpha_{n}}\right) y_{n, N}-P_{C}\left(I-\lambda_{n} \nabla f_{\alpha_{n}}\right) p\right\|^{2}
\end{aligned}
$$




$$
\begin{aligned}
& +2\left\langle P_{C}\left(I-\lambda_{n} \nabla f_{\alpha_{n}}\right) p-P_{C}\left(I-\lambda_{n} \nabla f\right) p, \tilde{y}_{n, N}-p\right\rangle \\
\leq & \left\|y_{n, N}-p\right\|^{2}+2\left\|P_{C}\left(I-\lambda_{n} \nabla f_{\alpha_{n}}\right) p-P_{C}\left(I-\lambda_{n} \nabla f\right) p\right\|\left\|\tilde{y}_{n, N}-p\right\| \\
\leq & \left\|x_{n}-p\right\|^{2}+2\left\|\left(I-\lambda_{n} \nabla f_{\alpha_{n}}\right) p-\left(I-\lambda_{n} \nabla f\right) p\right\|\left\|\tilde{y}_{n, N}-p\right\| \\
\leq & \left\|x_{n}-p\right\|^{2}+2 \lambda_{n} \alpha_{n}\|p\|\left\|\tilde{y}_{n, N}-p\right\| .
\end{aligned}
$$

Furthermore, utilizing Proposition 2.1 (ii), we have

$$
\begin{aligned}
\left\|t_{n}-p\right\|^{2} \leq & \left.\left.\| y_{n, N}-\lambda_{n} \nabla f_{\alpha_{n}}\left(\tilde{y}_{n, N}\right)\right)-p\left\|^{2}-\right\| y_{n, N}-\lambda_{n} \nabla f_{\alpha_{n}}\left(\tilde{y}_{n, N}\right)\right)-t_{n} \|^{2} \\
= & \left\|y_{n, N}-p\right\|^{2}-\left\|y_{n, N}-t_{n}\right\|^{2}+2 \lambda_{n}\left\langle\nabla f_{\alpha_{n}}\left(\tilde{y}_{n, N}\right), p-t_{n}\right\rangle \\
= & \left\|y_{n, N}-p\right\|^{2}-\left\|y_{n, N}-t_{n}\right\|^{2}+2 \lambda_{n}\left(\left\langle\nabla f_{\alpha_{n}}\left(\tilde{y}_{n, N}\right)-\nabla f_{\alpha_{n}}(p), p-\tilde{y}_{n, N}\right\rangle\right. \\
& \left.+\left\langle\nabla f_{\alpha_{n}}(p), p-\tilde{y}_{n, N}\right\rangle+\left\langle\nabla f_{\alpha_{n}}\left(\tilde{y}_{n, N}\right), \tilde{y}_{n, N}-t_{n}\right\rangle\right) \\
\leq & \left\|y_{n, N}-p\right\|^{2}-\left\|y_{n, N}-t_{n}\right\|^{2}+2 \lambda_{n}\left(\left\langle\nabla f_{\alpha_{n}}(p), p-\tilde{y}_{n, N}\right\rangle+\left\langle\nabla f_{\alpha_{n}}\left(\tilde{y}_{n, N}\right), \tilde{y}_{n, N}-t_{n}\right\rangle\right) \\
= & \left\|y_{n, N}-p\right\|^{2}-\left\|y_{n, N}-t_{n}\right\|^{2}+2 \lambda_{n}\left[\left\langle\left(\alpha_{n} I+\nabla f\right) p, p-\tilde{y}_{n, N}\right\rangle+\left\langle\nabla f_{\alpha_{n}}\left(\tilde{y}_{n, N}\right), \tilde{y}_{n, N}-t_{n}\right\rangle\right] \\
\leq & \left\|y_{n, N}-p\right\|^{2}-\left\|y_{n, N}-t_{n}\right\|^{2}+2 \lambda_{n}\left[\alpha_{n}\left\langle p, p-\tilde{y}_{n, N}\right\rangle+\left\langle\nabla f_{\alpha_{n}}\left(\tilde{y}_{n, N}\right), \tilde{y}_{n, N}-t_{n}\right\rangle\right] \\
= & \left\|y_{n, N}-p\right\|^{2}-\left\|y_{n, N}-\tilde{y}_{n, N}\right\|^{2}-2\left\langle y_{n, N}-\tilde{y}_{n, N}, \tilde{y}_{n, N}-t_{n}\right\rangle-\left\|\tilde{y}_{n, N}-t_{n}\right\|^{2} \\
& +2 \lambda_{n}\left[\alpha_{n}\left\langle p, p-\tilde{y}_{n, N}\right\rangle+\left\langle\nabla f_{\alpha_{n}}\left(\tilde{y}_{n, N}\right), \tilde{y}_{n, N}-t_{n}\right\rangle\right] \\
= & \left\|y_{n, N}-p\right\|^{2}-\left\|y_{n, N}-\tilde{y}_{n, N}\right\|^{2}-\left\|\tilde{y}_{n, N}-t_{n}\right\|^{2} \\
& +2\left\langle y_{n, N}-\lambda_{n} \nabla f_{\alpha_{n}}\left(\tilde{y}_{n, N}\right)-\tilde{y}_{n, N}, t_{n}-\tilde{y}_{n, N}\right\rangle+2 \lambda_{n} \alpha_{n}\left\langle p, p-\tilde{y}_{n, N}\right\rangle .
\end{aligned}
$$

In the meantime, by Proposition 2.1 (i), we have

$$
\begin{aligned}
& \left\langle y_{n, N}-\lambda_{n} \nabla f_{\alpha_{n}}\left(\tilde{y}_{n, N}\right)-\tilde{y}_{n, N}, t_{n}-\tilde{y}_{n, N}\right\rangle \\
& =\left\langle y_{n, N}-\lambda_{n} \nabla f_{\alpha_{n}}\left(y_{n, N}\right)-\tilde{y}_{n, N}, t_{n}-\tilde{y}_{n, N}\right\rangle+\left\langle\lambda_{n} \nabla f_{\alpha_{n}}\left(y_{n, N}\right)-\lambda_{n} \nabla f_{\alpha_{n}}\left(\tilde{y}_{n, N}\right), t_{n}-\tilde{y}_{n, N}\right\rangle \\
& \leq\left\langle\lambda_{n} \nabla f_{\alpha_{n}}\left(y_{n, N}\right)-\lambda_{n} \nabla f_{\alpha_{n}}\left(\tilde{y}_{n, N}\right), t_{n}-\tilde{y}_{n, N}\right\rangle \\
& \leq \lambda_{n}\left\|\nabla f_{\alpha_{n}}\left(y_{n, N}\right)-\nabla f_{\alpha_{n}}\left(\tilde{y}_{n, N}\right)\right\|\left\|t_{n}-\tilde{y}_{n, N}\right\| \\
& \leq \lambda_{n}\left(\alpha_{n}+\|A\|^{2}\right)\left\|y_{n, N}-\tilde{y}_{n, N}\right\|\left\|t_{n}-\tilde{y}_{n, N}\right\| .
\end{aligned}
$$

So, from (3.9) and (3.11), we obtain

$$
\begin{aligned}
\left\|t_{n}-p\right\|^{2} \leq & \left\|y_{n, N}-p\right\|^{2}-\left\|y_{n, N}-\tilde{y}_{n, N}\right\|^{2}-\left\|\tilde{y}_{n, N}-t_{n}\right\|^{2} \\
& +2\left\langle y_{n, N}-\lambda_{n} \nabla f_{\alpha_{n}}\left(\tilde{y}_{n, N}\right)-\tilde{y}_{n, N}, t_{n}-\tilde{y}_{n, N}\right\rangle+2 \lambda_{n} \alpha_{n}\left\langle p, p-\tilde{y}_{n, N}\right\rangle \\
\leq & \left\|y_{n, N}-p\right\|^{2}-\left\|y_{n, N}-\tilde{y}_{n, N}\right\|^{2}-\left\|\tilde{y}_{n, N}-t_{n}\right\|^{2} \\
& +2 \lambda_{n}\left(\alpha_{n}+\|A\|^{2}\right)\left\|y_{n, N}-\tilde{y}_{n, N}\right\|\left\|t_{n}-\tilde{y}_{n, N}\right\|+2 \lambda_{n} \alpha_{n}\left\langle p, p-\tilde{y}_{n, N}\right\rangle \\
\leq & \left\|y_{n, N}-p\right\|^{2}-\left\|y_{n, N}-\tilde{y}_{n, N}\right\|^{2}-\left\|\tilde{y}_{n, N}-t_{n}\right\|^{2} \\
& +\lambda_{n}^{2}\left(\alpha_{n}+\|A\|^{2}\right)^{2}\left\|y_{n, N}-\tilde{y}_{n, N}\right\|^{2}+\left\|\tilde{y}_{n, N}-t_{n}\right\|^{2}+2 \lambda_{n} \alpha_{n}\left\langle p, p-\tilde{y}_{n, N}\right\rangle
\end{aligned}
$$




$$
\begin{aligned}
= & \left\|y_{n, N}-p\right\|^{2}+2 \lambda_{n} \alpha_{n}\|p\|\left\|p-\tilde{y}_{n, N}\right\| \\
& +\left(\lambda_{n}^{2}\left(\alpha_{n}+\|A\|^{2}\right)^{2}-1\right)\left\|y_{n, N}-\tilde{y}_{n, N}\right\|^{2} \\
\leq & \left\|y_{n, N}-p\right\|^{2}+2 \lambda_{n} \alpha_{n}\|p\|\left\|\tilde{y}_{n, N}-p\right\| \\
\leq & \left\|y_{n, N}-p\right\|^{2}+2 \lambda_{n} \alpha_{n}\|p\|\left[\left\|y_{n, N}-p\right\|+\lambda_{n} \alpha_{n}\|p\|\right] \\
\leq & \left\|y_{n, N}-p\right\|^{2}+2 \sqrt{2} \lambda_{n} \alpha_{n}\|p\|\left\|y_{n, N}-p\right\|+2 \lambda_{n}^{2} \alpha_{n}^{2}\|p\|^{2} \\
= & \left(\left\|y_{n, N}-p\right\|+\sqrt{2} \lambda_{n} \alpha_{n}\|p\|\right)^{2} \\
\leq & \left(\left\|x_{n}-p\right\|+\sqrt{2} \lambda_{n} \alpha_{n}\|p\|\right)^{2} .
\end{aligned}
$$

Since $\left(\gamma_{n}+\sigma_{n}\right) \xi \leq \gamma_{n}$ for all $n \geq 0$, utilizing Lemmas 2.4 and 2.2 (b), from (3.9)-(3.11) and (3.15), we conclude that

$$
\begin{aligned}
& \left\|y_{n}-p\right\|^{2}=\left\|\beta_{n} x_{n}+\gamma_{n} z_{n}+\sigma_{n} T z_{n}-p\right\|^{2} \\
& =\left\|\beta_{n}\left(x_{n}-p\right)+\left(\gamma_{n}+\sigma_{n}\right) \frac{1}{\gamma_{n}+\sigma_{n}}\left[\gamma_{n}\left(z_{n}-p\right)+\sigma_{n}\left(T z_{n}-p\right)\right]\right\|^{2} \\
& =\beta_{n}\left\|x_{n}-p\right\|^{2}+\left(\gamma_{n}+\sigma_{n}\right)\left\|\frac{1}{\gamma_{n}+\sigma_{n}}\left[\gamma_{n}\left(z_{n}-p\right)+\sigma_{n}\left(T z_{n}-p\right)\right]\right\|^{2} \\
& -\beta_{n}\left(\gamma_{n}+\sigma_{n}\right)\left\|\frac{1}{\gamma_{n}+\sigma_{n}}\left[\gamma_{n}\left(z_{n}-x_{n}\right)+\sigma_{n}\left(T z_{n}-x_{n}\right)\right]\right\|^{2} \\
& \leq \beta_{n}\left\|x_{n}-p\right\|^{2}+\left(1-\beta_{n}\right)\left\|z_{n}-p\right\|^{2}-\frac{\beta_{n}}{1-\beta_{n}}\left\|y_{n}-x_{n}\right\|^{2} \\
& \leq \beta_{n}\left\|x_{n}-p\right\|^{2}+\left(1-\beta_{n}\right)\left\|t_{n}-p\right\|^{2}-\frac{\beta_{n}}{1-\beta_{n}}\left\|y_{n}-x_{n}\right\|^{2} \\
& \leq \beta_{n}\left\|x_{n}-p\right\|^{2}+\left(1-\beta_{n}\right)\left[\left\|y_{n, N}-p\right\|^{2}+2 \lambda_{n} \alpha_{n}\|p\|\left\|p-\tilde{y}_{n, N}\right\|\right. \\
& \left.+\left(\lambda_{n}^{2}\left(\alpha_{n}+\|A\|^{2}\right)^{2}-1\right)\left\|y_{n, N}-\tilde{y}_{n, N}\right\|^{2}\right]-\frac{\beta_{n}}{1-\beta_{n}}\left\|y_{n}-x_{n}\right\|^{2} \\
& \leq \beta_{n}\left\|x_{n}-p\right\|^{2}+\left(1-\beta_{n}\right)\left[\left\|x_{n}-p\right\|^{2}+2 \lambda_{n} \alpha_{n}\|p\|\left\|p-\tilde{y}_{n, N}\right\|\right. \\
& \left.+\left(\lambda_{n}^{2}\left(\alpha_{n}+\|A\|^{2}\right)^{2}-1\right)\left\|y_{n, N}-\tilde{y}_{n, N}\right\|^{2}\right]-\frac{\beta_{n}}{1-\beta_{n}}\left\|y_{n}-x_{n}\right\|^{2} \\
& \leq\left\|x_{n}-p\right\|^{2}+2 \lambda_{n} \alpha_{n}\|p\|\left\|p-\tilde{y}_{n, N}\right\| \\
& +\left(1-\beta_{n}\right)\left(\lambda_{n}^{2}\left(\alpha_{n}+\|A\|^{2}\right)^{2}-1\right)\left\|y_{n, N}-\tilde{y}_{n, N}\right\|^{2}-\frac{\beta_{n}}{1-\beta_{n}}\left\|y_{n}-x_{n}\right\|^{2} \\
& \leq\left\|x_{n}-p\right\|^{2}+2 \lambda_{n} \alpha_{n}\|p\|\left(\left\|x_{n}-p\right\|+\lambda_{n} \alpha_{n}\|p\|\right) \\
& +\left(1-\beta_{n}\right)\left(\lambda_{n}^{2}\left(\alpha_{n}+\|A\|^{2}\right)^{2}-1\right)\left\|y_{n, N}-\tilde{y}_{n, N}\right\|^{2}-\frac{\beta_{n}}{1-\beta_{n}}\left\|y_{n}-x_{n}\right\|^{2} \\
& \leq\left\|x_{n}-p\right\|^{2}+2\left\|x_{n}-p\right\|\left(\sqrt{2} \lambda_{n} \alpha_{n}\|p\|\right)+\left(\sqrt{2} \lambda_{n} \alpha_{n}\|p\|\right)^{2} \\
& +\left(1-\beta_{n}\right)\left(\lambda_{n}^{2}\left(\alpha_{n}+\|A\|^{2}\right)^{2}-1\right)\left\|y_{n, N}-\tilde{y}_{n, N}\right\|^{2}-\frac{\beta_{n}}{1-\beta_{n}}\left\|y_{n}-x_{n}\right\|^{2} \\
& =\left(\left\|x_{n}-p\right\|+\sqrt{2} \lambda_{n} \alpha_{n}\|p\|\right)^{2} \\
& +\left(1-\beta_{n}\right)\left(\lambda_{n}^{2}\left(\alpha_{n}+\|A\|^{2}\right)^{2}-1\right)\left\|y_{n, N}-\tilde{y}_{n, N}\right\|^{2}-\frac{\beta_{n}}{1-\beta_{n}}\left\|y_{n}-x_{n}\right\|^{2}
\end{aligned}
$$




$$
\leq\left(\left\|x_{n}-p\right\|+\sqrt{2} \lambda_{n} \alpha_{n}\|p\|\right)^{2}
$$

Noticing the boundedness of $\left\{S x_{n}\right\}$, we get $\sup _{n \geq 1}\left\|\gamma S x_{n}-\mu F p\right\| \leq \widetilde{M}$ for some $\widetilde{M}>0$. Moreover, utilizing Lemma 2.7 we have from (3.1)

$$
\begin{aligned}
& \left\|x_{n+1}-p\right\| \\
& =\left\|P_{C}\left[\epsilon_{n} \gamma\left(\delta_{n} V x_{n}+\left(1-\delta_{n}\right) S x_{n}\right)+\left(I-\epsilon_{n} \mu F\right) y_{n}\right]-P_{C} p\right\| \\
& \leq\left\|\epsilon_{n} \gamma\left(\delta_{n} V x_{n}+\left(1-\delta_{n}\right) S x_{n}\right)+\left(I-\epsilon_{n} \mu F\right) y_{n}-p\right\| \\
& =\left\|\epsilon_{n} \gamma\left(\delta_{n} V x_{n}+\left(1-\delta_{n}\right) S x_{n}\right)-\epsilon_{n} \mu F p+\left(I-\epsilon_{n} \mu F\right) y_{n}-\left(I-\epsilon_{n} \mu F\right) p\right\| \\
& \leq\left\|\epsilon_{n} \gamma\left(\delta_{n} V x_{n}+\left(1-\delta_{n}\right) S x_{n}\right)-\epsilon_{n} \mu F p\right\|+\left\|\left(I-\epsilon_{n} \mu F\right) y_{n}-\left(I-\epsilon_{n} \mu F\right) p\right\| \\
& =\epsilon_{n}\left\|\delta_{n}\left(\gamma V x_{n}-\mu F p\right)+\left(1-\delta_{n}\right)\left(\gamma S x_{n}-\mu F p\right)\right\|+\left\|\left(I-\epsilon_{n} \mu F\right) y_{n}-\left(I-\epsilon_{n} \mu F\right) p\right\| \\
& \leq \epsilon_{n}\left[\delta_{n}\left\|\gamma V x_{n}-\mu F p\right\|+\left(1-\delta_{n}\right)\left\|\gamma S x_{n}-\mu F p\right\|\right]+\left(1-\epsilon_{n} \tau\right)\left\|y_{n}-p\right\| \\
& \leq \epsilon_{n}\left[\delta_{n}\left(\left\|\gamma V x_{n}-\gamma V p\right\|+\|\gamma V p-\mu F p\|\right)+\left(1-\delta_{n}\right) \widetilde{M}\right]+\left(1-\epsilon_{n} \tau\right)\left\|y_{n}-p\right\| \\
& \leq \epsilon_{n}\left[\delta_{n} \gamma \rho\left\|x_{n}-p\right\|+\delta_{n}\|\gamma V p-\mu F p\|+\left(1-\delta_{n}\right) \widetilde{M}\right]+\left(1-\epsilon_{n} \tau\right)\left[\left\|x_{n}-p\right\|+\sqrt{2} \lambda_{n} \alpha_{n}\|p\|\right] \\
& \leq \epsilon_{n}\left[\delta_{n} \gamma \rho\left\|x_{n}-p\right\|+\max \{\widetilde{M},\|\gamma V p-\mu F p\|\}+\left(1-\epsilon_{n} \tau\right)\left[\left\|x_{n}-p\right\|+\sqrt{2} \lambda_{n} \alpha_{n}\|p\|\right]\right. \\
& \leq \epsilon_{n} \gamma \rho\left\|x_{n}-p\right\|+\epsilon_{n} \max \{\widetilde{M},\|\gamma V p-\mu F p\|\}+\left(1-\epsilon_{n} \tau\right)\left\|x_{n}-p\right\|+\sqrt{2} \lambda_{n} \alpha_{n}\|p\| \\
& =\left[1-(\tau-\gamma \rho) \epsilon_{n}\right]\left\|x_{n}-p\right\|+\epsilon_{n} \max \{\widetilde{M},\|\gamma V p-\mu F p\|\}+\sqrt{2} \lambda_{n} \alpha_{n}\|p\| \\
& =\left[1-(\tau-\gamma \rho) \epsilon_{n}\right]\left\|x_{n}-p\right\|+(\tau-\gamma \rho) \epsilon_{n} \max \left\{\frac{\widetilde{M}}{\tau-\gamma \rho}, \frac{\|\gamma V p-\mu F p\|}{\tau-\gamma \rho}\right\}+\sqrt{2} \lambda_{n} \alpha_{n}\|p\| \\
& \quad \leq \max \left\{\left\|x_{n}-p\right\|, \frac{\widetilde{M}}{\tau-\gamma \rho}, \frac{\|\gamma V p-\mu F p\|}{\tau-\gamma \rho}\right\}
\end{aligned}
$$

By induction, we can derive

$$
\left\|x_{n+1}-p\right\| \leq \max \left\{\left\|x_{0}-p\right\|, \frac{\widetilde{M}}{\tau-\gamma \rho}, \frac{\|\gamma V p-\mu F p\|}{\tau-\gamma \rho}\right\}+\sum_{k=0}^{n} \sqrt{2} \lambda_{k} \alpha_{k}\|p\|, \quad \forall n \geq 0 .
$$

Since $\left\{\lambda_{n}\right\} \subset[a, b] \subset\left(0, \frac{1}{\|A\|^{2}}\right)$ and $\sum_{n=0}^{\infty} \alpha_{n}<\infty$, we know that $\left\{x_{n}\right\}$ is bounded, and so are the sequences $\left\{u_{n}\right\},\left\{t_{n}\right\},\left\{\tilde{t}_{n}\right\},\left\{y_{n}\right\},\left\{\tilde{y}_{n, N}\right\},\left\{y_{n, i}\right\}$ for each $i=1, \ldots, N$. Since $\left\|T G t_{n}-p\right\| \leq \frac{1+\xi}{1-\xi} \| G t_{n}-$ $p\left\|\leq \frac{1+\xi}{1-\xi}\right\| t_{n}-p \|,\left\{T G t_{n}\right\}$ is also bounded.

Proposition 3.7. Let us suppose that $\Omega \neq \varnothing$. Moreover, let us suppose that the following hold:

(H0) $\lim _{n \rightarrow \infty} \delta_{n}=0, \lim _{n \rightarrow \infty} \epsilon_{n}=0$ and $\sum_{n=0}^{\infty} \epsilon_{n} \delta_{n}=\infty$;

(H1) $\lim _{n \rightarrow \infty} \frac{\delta_{n-1}}{\delta_{n}}=1$ and $\lim _{n \rightarrow \infty} \frac{\left|\alpha_{n}-\alpha_{n-1}\right|}{\epsilon_{n} \delta_{n}}=0$;

(H2) $\lim _{n \rightarrow \infty} \frac{\left|\beta_{n, i}-\beta_{n-1, i}\right|}{\epsilon_{n} \delta_{n}}=0$ for each $i=1, \ldots, N$;

(H3) $\lim _{n \rightarrow \infty} \frac{\left|\lambda_{n}-\lambda_{n-1}\right|}{\epsilon_{n} \delta_{n}}=0$ and $\lim _{n \rightarrow \infty} \frac{1}{\delta_{n}}\left|1-\frac{\epsilon_{n-1}}{\epsilon_{n}}\right|=0$;

(H4) $\lim _{n \rightarrow \infty} \frac{\left|r_{n}-r_{n-1}\right|}{\epsilon_{n} \delta_{n}}=0$; 
(H5) $\lim _{n \rightarrow \infty} \frac{\left|\beta_{n}-\beta_{n-1}\right|}{\epsilon_{n} \delta_{n}}=0$;

(H6) $\lim _{n \rightarrow \infty} \frac{1}{\epsilon_{n} \delta_{n}}\left|\frac{\gamma_{n}}{1-\beta_{n}}-\frac{\gamma_{n-1}}{1-\beta_{n-1}}\right|=0$.

If $\left\|u_{n}-u_{n-1}\right\|=o\left(\epsilon_{n} \delta_{n}\right)$, then $\lim _{n \rightarrow \infty}\left\|x_{n+1}-x_{n}\right\|=0$.

Proof. First, it is known that $\left\{\lambda_{n}\right\} \subset[a, b] \subset\left(0, \frac{1}{\|A\|^{2}}\right)$ and $\left\{\beta_{n}\right\} \subset[c, d] \subset(0,1)$ as in the proof of Proposition 3.6. Taking into account $\liminf _{n \rightarrow \infty} r_{n}>0$, we may assume, without loss of generality, that $\left\{r_{n}\right\} \subset[\bar{r}, \infty)$ for some $\bar{r}>0$. First, we write $y_{n-1}=\beta_{n-1} x_{n-1}+\left(1-\beta_{n-1}\right) w_{n-1}, \quad \forall n \geq$ 1 , where $w_{n-1}=\frac{y_{n-1}-\beta_{n-1} x_{n-1}}{1-\beta_{n-1}}$. It follows that for all $n \geq 1$

$$
\begin{aligned}
w_{n}-w_{n-1}= & \frac{y_{n}-\beta_{n} x_{n}}{1-\beta_{n}}-\frac{y_{n-1}-\beta_{n-1} x_{n-1}}{1-\beta_{n-1}} \\
= & \frac{\gamma_{n} z_{n}+\sigma_{n} T z_{n}}{1-\beta_{n}}-\frac{\gamma_{n-1} z_{n-1}+\sigma_{n-1} T z_{n-1}}{1-\beta_{n-1}} \\
= & \frac{\gamma_{n}\left(z_{n}-z_{n-1}\right)+\sigma_{n}\left(T z_{n}-T z_{n-1}\right)}{1-\beta_{n}}+\left(\frac{\gamma_{n}}{1-\beta_{n}}-\frac{\gamma_{n-1}}{1-\beta_{n-1}}\right) z_{n-1} \\
& +\left(\frac{\sigma_{n}}{1-\beta_{n}}-\frac{\sigma_{n-1}}{1-\beta_{n-1}}\right) T z_{n-1} .
\end{aligned}
$$

Since $\left(\gamma_{n}+\sigma_{n}\right) \xi \leq \gamma_{n}$ for all $n \geq 0$, utilizing Lemma 2.4 we have

$$
\left\|\gamma_{n}\left(z_{n}-z_{n-1}\right)+\sigma_{n}\left(T z_{n}-T z_{n-1}\right)\right\| \leq\left(\gamma_{n}+\sigma_{n}\right)\left\|z_{n}-z_{n-1}\right\|
$$

Next, we estimate $\left\|y_{n}-y_{n-1}\right\|$. Indeed, according to $\lambda_{n}\left(\alpha_{n}+\|A\|^{2}\right)<1$

$$
\begin{aligned}
& \left\|t_{n}-t_{n-1}\right\| \\
& \leq\left\|\left(y_{n, N}-\lambda_{n} \nabla f_{\alpha_{n}}\left(\tilde{y}_{n, N}\right)\right)-\left(y_{n-1, N}-\lambda_{n-1} \nabla f_{\alpha_{n-1}}\left(\tilde{y}_{n-1, N}\right)\right)\right\| \\
& \leq\left\|y_{n, N}-y_{n-1, N}\right\|+\left\|\lambda_{n} \nabla f_{\alpha_{n}}\left(\tilde{y}_{n, N}\right)-\lambda_{n-1} \nabla f_{\alpha_{n-1}}\left(\tilde{y}_{n-1, N}\right)\right\| \\
& \leq\left\|y_{n, N}-y_{n-1, N}\right\|+\left|\lambda_{n}-\lambda_{n-1}\right|\left\|\nabla f_{\alpha_{n}}\left(\tilde{y}_{n, N}\right)\right\|+\lambda_{n-1}\left\|\nabla f_{\alpha_{n}}\left(\tilde{y}_{n, N}\right)-\nabla f_{\alpha_{n-1}}\left(\tilde{y}_{n-1, N}\right)\right\| \\
& \leq\left\|y_{n, N}-y_{n-1, N}\right\|+\left|\lambda_{n}-\lambda_{n-1}\right|\left\|\nabla f_{\alpha_{n}}\left(\tilde{y}_{n, N}\right)\right\| \\
& +\lambda_{n-1}\left(\left\|\nabla f_{\alpha_{n}}\left(\tilde{y}_{n, N}\right)-\nabla f_{\alpha_{n-1}}\left(\tilde{y}_{n, N}\right)\right\|+\left\|\nabla f_{\alpha_{n-1}}\left(\tilde{y}_{n, N}\right)-\nabla f_{\alpha_{n-1}}\left(\tilde{y}_{n-1, N}\right)\right\|\right) \\
& \leq\left\|y_{n, N}-y_{n-1, N}\right\|+\left|\lambda_{n}-\lambda_{n-1}\right|\left\|\nabla f_{\alpha_{n}}\left(\tilde{y}_{n, N}\right)\right\| \\
& +\lambda_{n-1}\left[\left|\alpha_{n}-\alpha_{n-1}\right|\left\|\tilde{y}_{n, N}\right\|+\left(\alpha_{n-1}+\|A\|^{2}\right)\left\|\tilde{y}_{n, N}-\tilde{y}_{n-1, N}\right\|\right] \\
& =\left\|y_{n, N}-y_{n-1, N}\right\|+\left|\lambda_{n}-\lambda_{n-1}\right|\left\|\nabla f_{\alpha_{n}}\left(\tilde{y}_{n, N}\right)\right\| \\
& +\lambda_{n-1}\left|\alpha_{n}-\alpha_{n-1}\right|\left\|\tilde{y}_{n, N}\right\|+\lambda_{n-1}\left(\alpha_{n-1}+\|A\|^{2}\right)\left\|\tilde{y}_{n, N}-\tilde{y}_{n-1, N}\right\| \\
& \leq\left\|y_{n, N}-y_{n-1, N}\right\|+\left|\lambda_{n}-\lambda_{n-1}\right|\left\|\nabla f_{\alpha_{n}}\left(\tilde{y}_{n, N}\right)\right\|+\lambda_{n-1}\left|\alpha_{n}-\alpha_{n-1}\right|\left\|\tilde{y}_{n, N}\right\|+\left\|\tilde{y}_{n, N}-\tilde{y}_{n-1, N}\right\|, \\
& =\left\|P_{C}\left(y_{n, N}-\lambda_{n} \nabla f_{\alpha_{n}}\left(y_{n, N}\right)\right)-P_{C}\left(y_{n-1, N}-\lambda_{n-1} \nabla f_{\alpha_{n-1}}\left(y_{n-1, N}\right)\right)\right\|
\end{aligned}
$$




$$
\begin{aligned}
\leq & \left\|P_{C}\left(y_{n, N}-\lambda_{n} \nabla f_{\alpha_{n}}\left(y_{n, N}\right)\right)-P_{C}\left(y_{n-1, N}-\lambda_{n} \nabla f_{\alpha_{n}}\left(y_{n-1, N}\right)\right)\right\| \\
& +\left\|P_{C}\left(y_{n-1, N}-\lambda_{n} \nabla f_{\alpha_{n}}\left(y_{n-1, N}\right)\right)-P_{C}\left(y_{n-1, N}-\lambda_{n-1} \nabla f_{\alpha_{n-1}}\left(y_{n-1, N}\right)\right)\right\| \\
\leq & \left\|y_{n, N}-y_{n-1, N}\right\|+\left\|\left(y_{n-1, N}-\lambda_{n} \nabla f_{\alpha_{n}}\left(y_{n-1, N}\right)\right)-\left(y_{n-1, N}-\lambda_{n-1} \nabla f_{\alpha_{n-1}}\left(y_{n-1, N}\right)\right)\right\| \\
= & \left\|y_{n, N}-y_{n-1, N}\right\|+\left\|\lambda_{n} \nabla f_{\alpha_{n}}\left(y_{n-1, N}\right)-\lambda_{n-1} \nabla f_{\alpha_{n-1}}\left(y_{n-1, N}\right)\right\| \\
\leq & \left\|y_{n, N}-y_{n-1, N}\right\|+\left|\lambda_{n} \alpha_{n}-\lambda_{n-1} \alpha_{n-1}\right|\left\|y_{n-1, N}\right\|+\left|\lambda_{n}-\lambda_{n-1}\right|\left\|\nabla f\left(y_{n-1, N}\right)\right\| \\
\leq & \left\|y_{n, N}-y_{n-1, N}\right\|+\left(\alpha_{n}\left|\lambda_{n}-\lambda_{n-1}\right|+\lambda_{n-1}\left|\alpha_{n}-\alpha_{n-1}\right|\right)\left\|y_{n-1, N}\right\| \\
& +\left|\lambda_{n}-\lambda_{n-1}\right|\left\|\nabla f\left(y_{n-1, N}\right)\right\| .
\end{aligned}
$$

In the meantime, by the definition of $y_{n, i}$ one obtains that, for all $i=N, \ldots, 2$,

$\left\|y_{n, i}-y_{n-1, i}\right\| \leq \beta_{n, i}\left\|u_{n}-u_{n-1}\right\|+\left\|S_{i} u_{n-1}-y_{n-1, i-1}\right\|\left|\beta_{n, i}-\beta_{n-1, i}\right|+\left(1-\beta_{n, i}\right) \mid y_{n, i-1}-y_{n-1, i-1} \|$.

In the case $i=1$, we have

$$
\begin{aligned}
\left\|y_{n, 1}-y_{n-1,1}\right\| & \leq \beta_{n, 1}\left\|u_{n}-u_{n-1}\right\|+\left\|S_{1} u_{n-1}-u_{n-1}\right\|\left|\beta_{n, 1}-\beta_{n-1,1}\right|+\left(1-\beta_{n, 1}\right)\left\|u_{n}-u_{n-1}\right\| \\
& =\left\|u_{n}-u_{n-1}\right\|+\left\|S_{1} u_{n-1}-u_{n-1}\right\|\left|\beta_{n, 1}-\beta_{n-1,1}\right|
\end{aligned}
$$

Substituting (3.23) in all (3.22)-type one obtains for $i=2, \ldots, N$

$$
\begin{aligned}
\left\|y_{n, i}-y_{n-1, i}\right\| \leq & \left\|u_{n}-u_{n-1}\right\|+\sum_{k=2}^{i}\left\|S_{k} u_{n-1}-y_{n-1, k-1}\right\|\left|\beta_{n, k}-\beta_{n-1, k}\right| \\
& +\left\|S_{1} u_{n-1}-u_{n-1}\right\|\left|\beta_{n, 1}-\beta_{n-1,1}\right|,
\end{aligned}
$$

which together with (3.21), implies that

$$
\begin{aligned}
& \frac{\left\|\tilde{y}_{n, N}-\tilde{y}_{n-1, N}\right\|}{\epsilon_{n} \delta_{n}} \\
& \leq \frac{\left\|y_{n, N}-y_{n-1, N}\right\|}{\epsilon_{n} \delta_{n}}+\left(\alpha_{n} \frac{\left|\lambda_{n}-\lambda_{n-1}\right|}{\epsilon_{n} \delta_{n}}+\lambda_{n-1} \frac{\left|\alpha_{n}-\alpha_{n-1}\right|}{\epsilon_{n} \delta_{n}}\right)\left\|y_{n-1, N}\right\|+\frac{\left|\lambda_{n}-\lambda_{n-1}\right|}{\epsilon_{n} \delta_{n}}\left\|\nabla f\left(y_{n-1, N}\right)\right\| \\
& \leq \frac{\left\|u_{n}-u_{n-1}\right\|}{\epsilon_{n} \delta_{n}}+\sum_{k=2}^{N}\left\|S_{k} u_{n-1}-y_{n-1, k-1}\right\| \frac{\left|\beta_{n, k}-\beta_{n-1, k}\right|}{\epsilon_{n} \delta_{n}}+\left\|S_{1} u_{n-1}-u_{n-1}\right\| \frac{\left|\beta_{n, 1}-\beta_{n-1,1}\right|}{\epsilon_{n} \delta_{n}} \\
& \quad+\left(\alpha_{n} \frac{\left|\lambda_{n}-\lambda_{n-1}\right|}{\epsilon_{n} \delta_{n}}+\lambda_{n-1} \frac{\left|\alpha_{n}-\alpha_{n-1}\right|}{\epsilon_{n} \delta_{n}}\right)\left\|y_{n-1, N}\right\|+\frac{\left|\lambda_{n}-\lambda_{n-1}\right|}{\epsilon_{n} \delta_{n}}\left\|\nabla f\left(y_{n-1, N}\right)\right\| .
\end{aligned}
$$

Since $\left\|u_{n}-u_{n-1}\right\|=o\left(\epsilon_{n} \delta_{n}\right)$ and the sequences $\left\{u_{n}\right\},\left\{y_{n, i}\right\}_{i=1}^{N}$ are bounded, we know that

$$
\lim _{n \rightarrow \infty} \frac{\left\|\tilde{y}_{n, N}-\tilde{y}_{n-1, N}\right\|}{\epsilon_{n} \delta_{n}}=0 .
$$

Combining (3.18)-(3.20) and (3.24) we conclude from the nonexpansivity of $G$ that

$$
\left\|w_{n}-w_{n-1}\right\|
$$




$$
\begin{aligned}
\leq & \frac{\left\|\gamma_{n}\left(z_{n}-z_{n-1}\right)+\sigma_{n}\left(T z_{n}-T z_{n-1}\right)\right\|}{1-\beta_{n}}+\left|\frac{\gamma_{n}}{1-\beta_{n}}-\frac{\gamma_{n-1}}{1-\beta_{n-1}}\right|\left\|z_{n-1}\right\| \\
& +\left|\frac{\sigma_{n}}{1-\beta_{n}}-\frac{\sigma_{n-1}}{1-\beta_{n-1}}\right|\left\|T z_{n-1}\right\| \\
\leq & \frac{\left(\gamma_{n}+\sigma_{n}\right)\left\|z_{n}-z_{n-1}\right\|}{1-\beta_{n}}+\left|\frac{\gamma_{n}}{1-\beta_{n}}-\frac{\gamma_{n-1}}{1-\beta_{n-1}}\right|\left(\left\|z_{n-1}\right\|+\left\|T z_{n-1}\right\|\right) \\
\leq & \left\|t_{n}-t_{n-1}\right\|+\left|\frac{\gamma_{n}}{1-\beta_{n}}-\frac{\gamma_{n-1}}{1-\beta_{n-1}}\right|\left(\left\|z_{n-1}\right\|+\left\|T z_{n-1}\right\|\right) \\
\leq & \left\|y_{n, N}-y_{n-1, N}\right\|+\left|\lambda_{n}-\lambda_{n-1}\right|\left\|\nabla f_{\alpha_{n}}\left(\tilde{y}_{n, N}\right)\right\|+\lambda_{n-1}\left|\alpha_{n}-\alpha_{n-1}\right|\left\|\tilde{y}_{n, N}\right\|+\left\|\tilde{y}_{n, N}-\tilde{y}_{n-1, N}\right\| \\
& +\left|\frac{\gamma_{n}}{1-\beta_{n}}-\frac{\gamma_{n-1}}{1-\beta_{n-1}}\right|\left(\left\|z_{n-1}\right\|+\left\|T z_{n-1}\right\|\right) \\
\leq & \left\|u_{n}-u_{n-1}\right\|+\sum_{k=2}^{N}\left\|S_{k} u_{n-1}-y_{n-1, k-1}\right\|\left|\beta_{n, k}-\beta_{n-1, k}\right|+\left\|S_{1} u_{n-1}-u_{n-1}\right\|\left|\beta_{n, 1}-\beta_{n-1,1}\right| \\
& +\left|\lambda_{n}-\lambda_{n-1}\right|\left\|\nabla f_{\alpha_{n}}\left(\tilde{y}_{n, N}\right)\right\|+\lambda_{n-1}\left|\alpha_{n}-\alpha_{n-1}\right|\left\|\tilde{y}_{n, N}\right\|+\left\|\tilde{y}_{n, N}-\tilde{y}_{n-1, N}\right\| \\
& +\left|\frac{\gamma_{n}}{1-\beta_{n}}-\frac{\gamma_{n-1}}{1-\beta_{n-1}}\right|\left(\left\|z_{n-1}\right\|+\left\|T z_{n-1}\right\|\right) .
\end{aligned}
$$

Moreover, by Lemma 2.10, we know that

$$
\left\|u_{n}-u_{n-1}\right\| \leq\left\|x_{n}-x_{n-1}\right\|+L\left|1-\frac{r_{n-1}}{r_{n}}\right|,
$$

where $L=\sup _{n \geq 0}\left\|u_{n}-x_{n}\right\|$. Further, we observe that

$$
\left\{\begin{array}{l}
y_{n}=\beta_{n} x_{n}+\left(1-\beta_{n}\right) w_{n}, \\
y_{n-1}=\beta_{n-1} x_{n-1}+\left(1-\beta_{n-1}\right) w_{n-1},
\end{array} \quad \forall n \geq 1 .\right.
$$

Simple calculations show that

$$
y_{n}-y_{n-1}=\left(1-\beta_{n}\right)\left(w_{n}-w_{n-1}\right)+\left(\beta_{n}-\beta_{n-1}\right)\left(x_{n-1}-w_{n-1}\right)+\beta_{n}\left(x_{n}-x_{n-1}\right) .
$$

Consequently, passing to the norm we get from (3.26) and (3.27)

$$
\begin{aligned}
&\left\|y_{n}-y_{n-1}\right\| \\
& \leq\left(1-\beta_{n}\right)\left\|w_{n}-w_{n-1}\right\|+\left|\beta_{n}-\beta_{n-1}\right|\left\|x_{n-1}-w_{n-1}\right\|+\beta_{n}\left\|x_{n}-x_{n-1}\right\| \\
& \leq\left(1-\beta_{n}\right)\left[\left\|u_{n}-u_{n-1}\right\|+\sum_{k=2}^{N}\left\|S_{k} u_{n-1}-y_{n-1, k-1}\right\|\left|\beta_{n, k}-\beta_{n-1, k}\right|\right. \\
&+\left\|S_{1} u_{n-1}-u_{n-1}\right\|\left|\beta_{n, 1}-\beta_{n-1,1}\right|+\left|\lambda_{n}-\lambda_{n-1}\right|\left\|\nabla f_{\alpha_{n}}\left(\tilde{y}_{n, N}\right)\right\|+\lambda_{n-1}\left|\alpha_{n}-\alpha_{n-1}\right|\left\|\tilde{y}_{n, N}\right\| \\
&\left.+\left\|\tilde{y}_{n, N}-\tilde{y}_{n-1, N}\right\|+\left|\frac{\gamma_{n}}{1-\beta_{n}}-\frac{\gamma_{n-1}}{1-\beta_{n-1}}\right|\left(\left\|z_{n-1}\right\|+\left\|T z_{n-1}\right\|\right)\right] \\
&+\left|\beta_{n}-\beta_{n-1}\right|\left\|x_{n-1}-w_{n-1}\right\|+\beta_{n}\left\|x_{n}-x_{n-1}\right\| \\
& \leq\left(1-\beta_{n}\right)\left[\left\|x_{n}-x_{n-1}\right\|+L\left|1-\frac{r_{n-1}}{r_{n}}\right|+\sum_{k=2}^{N}\left\|S_{k} u_{n-1}-y_{n-1, k-1}\right\|\left|\beta_{n, k}-\beta_{n-1, k}\right|\right. \\
&+\left\|S_{1} u_{n-1}-u_{n-1}\right\|\left|\beta_{n, 1}-\beta_{n-1,1}\right|+\left|\lambda_{n}-\lambda_{n-1}\right|\left\|\nabla f_{\alpha_{n}}\left(\tilde{y}_{n, N}\right)\right\|+\lambda_{n-1}\left|\alpha_{n}-\alpha_{n-1}\right|\left\|\tilde{y}_{n, N}\right\|
\end{aligned}
$$




$$
\begin{aligned}
& \left.+\left\|\tilde{y}_{n, N}-\tilde{y}_{n-1, N}\right\|+\left|\frac{\gamma_{n}}{1-\beta_{n}}-\frac{\gamma_{n-1}}{1-\beta_{n-1}}\right|\left(\left\|z_{n-1}\right\|+\left\|T z_{n-1}\right\|\right)\right] \\
& +\left|\beta_{n}-\beta_{n-1}\right|\left\|x_{n-1}-w_{n-1}\right\|+\beta_{n}\left\|x_{n}-x_{n-1}\right\| \\
\leq & \left\|x_{n}-x_{n-1}\right\|+L \frac{\left|r_{n}-r_{n-1}\right|}{r_{n}}+\sum_{k=2}^{N}\left\|S_{k} u_{n-1}-y_{n-1, k-1}\right\|\left|\beta_{n, k}-\beta_{n-1, k}\right| \\
& +\left\|S_{1} u_{n-1}-u_{n-1}\right\|\left|\beta_{n, 1}-\beta_{n-1,1}\right|+\left|\lambda_{n}-\lambda_{n-1}\right|\left\|\nabla f_{\alpha_{n}}\left(\tilde{y}_{n, N}\right)\right\|+b\left|\alpha_{n}-\alpha_{n-1}\right|\left\|\tilde{y}_{n, N}\right\| \\
& +\left\|\tilde{y}_{n, N}-\tilde{y}_{n-1, N}\right\|+\left|\frac{\gamma_{n}}{1-\beta_{n}}-\frac{\gamma_{n-1}}{1-\beta_{n-1}}\right|\left(\left\|z_{n-1}\right\|+\left\|T z_{n-1}\right\|\right)+\left|\beta_{n}-\beta_{n-1}\right|\left\|x_{n-1}-w_{n-1}\right\| \\
\leq & \left\|x_{n}-x_{n-1}\right\|+L \frac{\left|r_{n}-r_{n-1}\right|}{\bar{r}}+\sum_{k=2}^{N}\left\|S_{k} u_{n-1}-y_{n-1, k-1}\right\|\left|\beta_{n, k}-\beta_{n-1, k}\right| \\
& +\left\|S_{1} u_{n-1}-u_{n-1}\right\|\left|\beta_{n, 1}-\beta_{n-1,1}\right|+\left|\lambda_{n}-\lambda_{n-1}\right|\left\|\nabla f_{\alpha_{n}}\left(\tilde{y}_{n, N}\right)\right\|+b\left|\alpha_{n}-\alpha_{n-1}\right|\left\|\tilde{y}_{n, N}\right\| \\
& +\left\|\tilde{y}_{n, N}-\tilde{y}_{n-1, N}\right\|+\left|\frac{\gamma_{n}}{1-\beta_{n}}-\frac{\gamma_{n-1}}{1-\beta_{n-1}}\right|\left(\left\|z_{n-1}\right\|+\left\|T z_{n-1}\right\|\right)+\left|\beta_{n}-\beta_{n-1}\right|\left\|x_{n-1}-w_{n-1}\right\| \\
\leq & \left\|x_{n}-x_{n-1}\right\|+\widetilde{M}_{0}\left[\frac{\left|r_{n}-r_{n-1}\right|}{\bar{r}}+\sum_{k=1}^{N}\left|\beta_{n, k}-\beta_{n-1, k}\right|+\left|\lambda_{n}-\lambda_{n-1}\right|\right. \\
& \left.+\left|\alpha_{n}-\alpha_{n-1}\right|+\left|\beta_{n}-\beta_{n-1}\right|+\left|\frac{\gamma_{n}}{1-\beta_{n}}-\frac{\gamma_{n-1}}{1-\beta_{n-1}}\right|+\left\|\tilde{y}_{n, N}-\tilde{y}_{n-1, N}\right\|\right],
\end{aligned}
$$

where there exists a constant $\widetilde{M}_{0}>0$ such that

$$
\begin{aligned}
\sup _{n \geq 0}\{L & +1+\sum_{k=2}^{N}\left\|S_{k} u_{n}-y_{n, k-1}\right\|+\left\|S_{1} u_{n}-u_{n}\right\| \\
& \left.+\left\|\nabla f_{\alpha_{n}}\left(\tilde{y}_{n, N}\right)\right\|+b\left\|\tilde{y}_{n, N}\right\|+\left\|z_{n}\right\|+\left\|T z_{n}\right\|+\left\|x_{n}-w_{n}\right\|\right\} \leq \widetilde{M}_{0} .
\end{aligned}
$$

On the other hand, putting $\boldsymbol{\Lambda}_{n}=\delta_{n} V x_{n}+\left(1-\delta_{n}\right) S x_{n}$, we get

$$
\left\{\begin{array}{l}
v_{n}=\epsilon_{n} \gamma \boldsymbol{\Lambda}_{n}+\left(I-\epsilon_{n} \mu F\right) y_{n}, \\
v_{n-1}=\epsilon_{n-1} \gamma \boldsymbol{\Lambda}_{n-1}+\left(I-\epsilon_{n-1} \mu F\right) y_{n-1},
\end{array} \quad \forall n \geq 1 .\right.
$$

Simple calculations show that

$$
\boldsymbol{\Lambda}_{n}-\boldsymbol{\Lambda}_{n-1}=\delta_{n}\left(V x_{n}-V x_{n-1}\right)+\left(1-\delta_{n}\right)\left(S x_{n}-S x_{n-1}\right)+\left(\delta_{n}-\delta_{n-1}\right)\left(V x_{n-1}-S x_{n-1}\right),
$$

and

$$
v_{n}-v_{n-1}=\left(I-\epsilon_{n} \mu F\right) y_{n}-\left(I-\epsilon_{n} \mu F\right) y_{n-1}+\left(\epsilon_{n}-\epsilon_{n-1}\right)\left(\gamma \boldsymbol{\Lambda}_{n-1}-\mu F y_{n-1}\right)+\epsilon_{n} \gamma\left(\boldsymbol{\Lambda}_{n}-\boldsymbol{\Lambda}_{n-1}\right) .
$$

Then, passing to the norm we obtain from (3.28) that

$$
\begin{aligned}
\left\|\boldsymbol{\Lambda}_{n}-\boldsymbol{\Lambda}_{n-1}\right\| & \leq \delta_{n}\left\|V x_{n}-V x_{n-1}\right\|+\left(1-\delta_{n}\right)\left\|S x_{n}-S x_{n-1}\right\|+\left|\delta_{n}-\delta_{n-1}\right|\left\|V x_{n-1}-S x_{n-1}\right\| \\
& \leq \delta_{n} \rho\left\|x_{n}-x_{n-1}\right\|+\left(1-\delta_{n}\right)\left\|x_{n}-x_{n-1}\right\|+\left|\delta_{n}-\delta_{n-1}\right|\left\|V x_{n-1}-S x_{n-1}\right\| \\
& =\left(1-\delta_{n}(1-\rho)\right)\left\|x_{n}-x_{n-1}\right\|+\left|\delta_{n}-\delta_{n-1}\right|\left\|V x_{n-1}-S x_{n-1}\right\|,
\end{aligned}
$$


and hence

$$
\begin{aligned}
&\left\|x_{n+1}-x_{n}\right\| \leq\left\|v_{n}-v_{n-1}\right\| \\
& \leq\left\|\left(I-\epsilon_{n} \mu F\right) y_{n}-\left(I-\epsilon_{n} \mu F\right) y_{n-1}\right\|+\left|\epsilon_{n}-\epsilon_{n-1}\right|\left\|\gamma \boldsymbol{\Lambda}_{n-1}-\mu F y_{n-1}\right\|+\epsilon_{n} \gamma\left\|\boldsymbol{\Lambda}_{n}-\boldsymbol{\Lambda}_{n-1}\right\| \\
& \leq\left(1-\epsilon_{n} \tau\right)\left\|y_{n}-y_{n-1}\right\|+\left|\epsilon_{n}-\epsilon_{n-1}\right|\left\|\gamma \boldsymbol{\Lambda}_{n-1}-\mu F y_{n-1}\right\|+\epsilon_{n} \gamma\left\|\boldsymbol{\Lambda}_{n}-\boldsymbol{\Lambda}_{n-1}\right\| \\
& \leq\left(1-\epsilon_{n} \tau\right)\left\{\left\|x_{n}-x_{n-1}\right\|+\widetilde{M}_{0}\left[\frac{\left|r_{n}-r_{n-1}\right|}{\bar{r}}+\sum_{k=1}^{N}\left|\beta_{n, k}-\beta_{n-1, k}\right|+\left|\lambda_{n}-\lambda_{n-1}\right|\right.\right. \\
&+\left|\alpha_{n}-\alpha_{n-1}\right|+\left|\beta_{n}-\beta_{n-1}\right|+\mid \frac{\gamma_{n}}{1-\beta_{n}}-\frac{\gamma_{n-1}}{\left.\left.1-\beta_{n-1} \mid+\left\|\tilde{y}_{n, N}-\tilde{y}_{n-1, N}\right\|\right]\right\}} \\
&+\left|\epsilon_{n}-\epsilon_{n-1}\right|\left\|\gamma \boldsymbol{\Lambda}_{n-1}-\mu F y_{n-1}\right\|+\epsilon_{n} \gamma\left[\left(1-\delta_{n}(1-\rho)\right)\left\|x_{n}-x_{n-1}\right\|\right. \\
&\left.+\left|\delta_{n}-\delta_{n-1}\right|\left\|V x_{n-1}-S x_{n-1}\right\|\right] \\
& \leq\left(1-\epsilon_{n}(\tau-\gamma)-\epsilon_{n} \delta_{n}(1-\rho) \gamma\right)\left\|x_{n}-x_{n-1}\right\|+\widetilde{M}_{0}\left[\frac{\left|r_{n}-r_{n-1}\right|}{\bar{r}}+\sum_{k=1}^{N}\left|\beta_{n, k}-\beta_{n-1, k}\right|\right. \\
&\left.+\left|\lambda_{n}-\lambda_{n-1}\right|+\left|\alpha_{n}-\alpha_{n-1}\right|+\left|\beta_{n}-\beta_{n-1}\right|+\left|\frac{\gamma_{n}}{1-\beta_{n}}-\frac{\gamma_{n-1}}{1-\beta_{n-1}}\right|+\left\|\tilde{y}_{n, N}-\tilde{y}_{n-1, N}\right\|\right] \\
&+\left|\epsilon_{n}-\epsilon_{n-1}\right|\left\|\gamma \boldsymbol{\Lambda}_{n-1}-\mu F y_{n-1}\right\|+\epsilon_{n} \gamma\left|\delta_{n}-\delta_{n-1}\right|\left\|V x_{n-1}-S x_{n-1}\right\| \\
& \leq\left(1-\epsilon_{n} \delta_{n}(1-\rho) \gamma\right)\left\|x_{n}-x_{n-1}\right\|+\widetilde{M}_{1}\left[\frac{\left|r_{n}-r_{n-1}\right|}{\bar{r}}+\sum_{k=1}^{N}\left|\beta_{n, k}-\beta_{n-1, k}\right|\right. \\
&+\left|\lambda_{n}-\lambda_{n-1}\right|+\left|\alpha_{n}-\alpha_{n-1}\right|+\left|\beta_{n}-\beta_{n-1}\right|+\mid \frac{\gamma_{n}}{1-\beta_{n}}-\frac{\gamma_{n-1}}{1-\beta_{n-1} \mid} \\
&\left.+\left|\epsilon_{n}-\epsilon_{n-1}\right|+\epsilon_{n}\left|\delta_{n}-\delta_{n-1}\right|+\left\|\tilde{y}_{n, N}-\tilde{y}_{n-1, N}\right\|\right],
\end{aligned}
$$

where $\sup _{n \geq 0}\left\{\widetilde{M}_{0}+\left\|\gamma \boldsymbol{\Lambda}_{n}-\mu F y_{n}\right\|+\gamma\left\|V x_{n}-S x_{n}\right\|\right\} \leq \widetilde{M}_{1}$ for some $\widetilde{M}_{1}>0$. Noticing $\lim _{n \rightarrow \infty}$ $\frac{\left\|\tilde{y}_{n, N}-\tilde{y}_{n-1, N}\right\|}{\epsilon_{n} \delta_{n}}=0$ and using hypotheses (H0)-(H6) and Lemma 2.8, we obtain the claim.

Proposition 3.8. Let us suppose that $\Omega \neq \varnothing$. Let us suppose that $\left\{x_{n}\right\}$ is asymptotically regular. Then $\left\|x_{n}-u_{n}\right\|=\left\|x_{n}-T_{r_{n}} x_{n}\right\| \rightarrow 0$ and $\left\|y_{n, N}-\tilde{y}_{n, N}\right\| \rightarrow 0$ as $n \rightarrow \infty$.

Proof. Take a fixed $p \in \Omega$ arbitrarily. We recall that, by the firm nonexpansivity of $T_{r_{n}}$, a standard calculation (see [33]) shows that for $p \in \operatorname{GMEP}(\boldsymbol{\Theta}, h)$

$$
\left\|u_{n}-p\right\|^{2} \leq\left\|x_{n}-p\right\|^{2}-\left\|x_{n}-u_{n}\right\|^{2}
$$

Since $\left(\gamma_{n}+\delta_{n}\right) \xi \leq \gamma_{n}$ for all $n \geq 0$, utilizing Lemma 2.4 we have from (3.10), (3.15), (3.16) and (3.30) that

$$
\begin{aligned}
\left\|y_{n}-p\right\|^{2} \leq & \beta_{n}\left\|x_{n}-p\right\|^{2}+\left(1-\beta_{n}\right)\left\|z_{n}-p\right\|^{2}-\frac{\beta_{n}}{1-\beta_{n}}\left\|y_{n}-x_{n}\right\|^{2} \\
\leq & \beta_{n}\left\|x_{n}-p\right\|^{2}+\left(1-\beta_{n}\right)\left[\left\|t_{n}-p\right\|^{2}+v_{2}\left(v_{2}-2 \zeta_{2}\right)\left\|F_{2} t_{n}-F_{2} p\right\|^{2}\right. \\
& \left.+v_{1}\left(v_{1}-2 \zeta_{1}\right)\left\|F_{1} \tilde{t}_{n}-F_{1} \tilde{p}\right\|^{2}\right]-\frac{\beta_{n}}{1-\beta_{n}}\left\|y_{n}-x_{n}\right\|^{2}
\end{aligned}
$$




$$
\begin{aligned}
\leq & \beta_{n}\left\|x_{n}-p\right\|^{2}+\left(1-\beta_{n}\right)\left[\left\|y_{n, N}-p\right\|^{2}+2 \lambda_{n} \alpha \text { }\|p\|\left\|p-\tilde{y}_{n, N}\right\|\right. \\
& +\left(\lambda_{n}^{2}\left(\alpha_{n}+\|A\|^{2}\right)^{2}-1\right)\left\|y_{n, N}-\tilde{y}_{n, N}\right\|^{2}+v_{2}\left(v_{2}-2 \zeta_{2}\right)\left\|F_{2} t_{n}-F_{2} p\right\|^{2} \\
& \left.+v_{1}\left(v_{1}-2 \zeta_{1}\right)\left\|F_{1} \tilde{t}_{n}-F_{1} \tilde{p}\right\|^{2}\right]-\frac{\beta_{n}}{1-\beta_{n}}\left\|y_{n}-x_{n}\right\|^{2} \\
\leq & \beta_{n}\left\|x_{n}-p\right\|^{2}+\left(1-\beta_{n}\right)\left[\left\|u_{n}-p\right\|^{2}+2 \lambda_{n} \alpha_{n}\|p\|\left\|p-\tilde{y}_{n, N}\right\|\right. \\
& +\left(\lambda_{n}^{2}\left(\alpha_{n}+\|A\|^{2}\right)^{2}-1\right)\left\|y_{n, N}-\tilde{y}_{n, N}\right\|^{2}+v_{2}\left(v_{2}-2 \zeta_{2}\right)\left\|F_{2} t_{n}-F_{2} p\right\|^{2} \\
& \left.+v_{1}\left(v_{1}-2 \zeta_{1}\right)\left\|F_{1} \tilde{t}_{n}-F_{1} \tilde{p}\right\|^{2}\right]-\frac{\beta_{n}}{1-\beta_{n}}\left\|y_{n}-x_{n}\right\|^{2} \\
\leq & \beta_{n}\left\|x_{n}-p\right\|^{2}+\left(1-\beta_{n}\right)\left[\left\|x_{n}-p\right\|^{2}-\left\|x_{n}-u_{n}\right\|^{2}+2 \lambda_{n} \alpha_{n}\|p\|\left\|p-\tilde{y}_{n, N}\right\|\right. \\
& +\left(\lambda_{n}^{2}\left(\alpha_{n}+\|A\|^{2}\right)^{2}-1\right)\left\|y_{n, N}-\tilde{y}_{n, N}\right\|^{2}+v_{2}\left(v_{2}-2 \zeta_{2}\right)\left\|F_{2} t_{n}-F_{2} p\right\|^{2} \\
& \left.+v_{1}\left(v_{1}-2 \zeta_{1}\right)\left\|F_{1} \tilde{t}_{n}-F_{1} \tilde{p}\right\|^{2}\right]-\frac{\beta_{n}}{1-\beta_{n}}\left\|y_{n}-x_{n}\right\|^{2} \\
\leq & \left\|x_{n}-p\right\|^{2}-\left(1-\beta_{n}\right)\left[\left\|x_{n}-u_{n}\right\|^{2}+\left(1-\lambda_{n}^{2}\left(\alpha_{n}+\|A\|^{2}\right)^{2}\right)\left\|y_{n, N}-\tilde{y}_{n, N}\right\|^{2}\right. \\
& \left.+v_{2}\left(2 \zeta_{2}-v_{2}\right)\left\|F_{2} t_{n}-F_{2} p\right\|^{2}+v_{1}\left(2 \zeta_{1}-v_{1}\right)\left\|F_{1} \tilde{t}_{n}-F_{1} \tilde{p}\right\|^{2}\right] \\
& -\frac{\beta_{n}}{1-\beta_{n}}\left\|y_{n}-x_{n}\right\|^{2}+2 \lambda_{n} \alpha_{n}\|p\|\left\|p-\tilde{y}_{n, N}\right\| .
\end{aligned}
$$

Utilizing Lemmas 2.1 and 2.7, we obtain from $0<\gamma \leq \tau$, (3.1) and the last inequality that

$$
\begin{aligned}
\left\|x_{n+1}-p\right\|^{2} \leq & \left\|v_{n}-p\right\|^{2} \\
= & \left\|\left(I-\epsilon_{n} \mu F\right) y_{n}-\left(I-\epsilon_{n} \mu F\right) p+\epsilon_{n}\left[\gamma\left(\delta_{n} V x_{n}+\left(1-\delta_{n}\right) S x_{n}\right)-\mu F p\right]\right\|^{2} \\
\leq & {\left[\left\|\left(I-\epsilon_{n} \mu F\right) y_{n}-\left(I-\epsilon_{n} \mu F\right) p\right\|+\epsilon_{n}\left\|\gamma\left(\delta_{n} V x_{n}+\left(1-\delta_{n}\right) S x_{n}\right)-\mu F p\right\|\right]^{2} } \\
\leq & {\left[\left(1-\epsilon_{n} \tau\right)\left\|y_{n}-p\right\|+\epsilon_{n}\left\|\gamma\left(\delta_{n} V x_{n}+\left(1-\delta_{n}\right) S x_{n}\right)-\mu F p\right\|\right]^{2} } \\
= & {\left[\left(1-\epsilon_{n} \tau\right)\left\|y_{n}-p\right\|+\epsilon_{n} \tau \frac{1}{\tau}\left\|\gamma\left(\delta_{n} V x_{n}+\left(1-\delta_{n}\right) S x_{n}\right)-\mu F p\right\|\right]^{2} } \\
\leq & \left(1-\epsilon_{n} \tau\right)\left\|y_{n}-p\right\|^{2}+\epsilon_{n} \tau \frac{1}{\tau^{2}}\left\|\gamma\left(\delta_{n} V x_{n}+\left(1-\delta_{n}\right) S x_{n}\right)-\mu F p\right\|^{2} \\
\leq & \left(1-\epsilon_{n} \tau\right)\left\{\left\|x_{n}-p\right\|^{2}-\left(1-\beta_{n}\right)\left[\left\|x_{n}-u_{n}\right\|^{2}+\left(1-\lambda_{n}^{2}\left(\alpha_{n}+\|A\|^{2}\right)^{2}\right)\left\|y_{n, N}-\tilde{y}_{n, N}\right\|^{2}\right.\right. \\
& \left.+v_{2}\left(2 \zeta_{2}-v_{2}\right)\left\|F_{2} t_{n}-F_{2} p\right\|^{2}+v_{1}\left(2 \zeta_{1}-v_{1}\right)\left\|F_{1} \tilde{t}_{n}-F_{1} \tilde{p}\right\|^{2}\right]-\frac{\beta_{n}}{1-\beta_{n}}\left\|y_{n}-x_{n}\right\|^{2} \\
& \left.+2 \lambda_{n} \alpha_{n}\|p\|\left\|p-\tilde{y}_{n, N}\right\|\right\}+\epsilon_{n} \tau \frac{1}{\tau^{2}}\left\|\gamma\left(\delta_{n} V x_{n}+\left(1-\delta_{n}\right) S x_{n}\right)-\mu F p\right\|^{2} \\
\leq & \left\|x_{n}-p\right\|^{2}-\left(1-\epsilon_{n} \tau\right)\left\{( 1 - \beta _ { n } ) \left[\left\|x_{n}-u_{n}\right\|^{2}+\left(1-\lambda_{n}^{2}\left(\alpha_{n}+\|A\|^{2}\right)^{2}\right)\left\|y_{n, N}-\tilde{y}_{n, N}\right\|^{2}\right.\right. \\
& \left.\left.+v_{2}\left(2 \zeta_{2}-v_{2}\right)\left\|F_{2} t_{n}-F_{2} p\right\|^{2}+v_{1}\left(2 \zeta_{1}-v_{1}\right)\left\|F_{1} \tilde{t}_{n}-F_{1} \tilde{p}\right\|^{2}\right]+\frac{\beta_{n}}{1-\beta_{n}}\left\|y_{n}-x_{n}\right\|^{2}\right\} \\
& +2 \lambda_{n} \alpha_{n}\|p\|\left\|p-\tilde{y}_{n, N}\right\|+\epsilon_{n} \frac{1}{\tau}\left\|\gamma\left(\delta_{n} V x_{n}+\left(1-\delta_{n}\right) S x_{n}\right)-\mu F p\right\|^{2},
\end{aligned}
$$

which together with $\left\{\lambda_{n}\right\} \subset[a, b] \subset\left(0, \frac{1}{\|A\|^{2}}\right)$ and $\left\{\beta_{n}\right\} \subset[c, d] \subset(0,1)$, implies that

$$
\left(1-\epsilon_{n} \tau\right)\left\{( 1 - d ) \left[\left\|x_{n}-u_{n}\right\|^{2}+\left(1-b^{2}\left(\alpha_{n}+\|A\|^{2}\right)^{2}\right)\left\|y_{n, N}-\tilde{y}_{n, N}\right\|^{2}\right.\right.
$$




$$
\begin{aligned}
& \left.\left.+v_{2}\left(2 \zeta_{2}-v_{2}\right)\left\|F_{2} t_{n}-F_{2} p\right\|^{2}+v_{1}\left(2 \zeta_{1}-v_{1}\right)\left\|F_{1} \tilde{t}_{n}-F_{1} \tilde{p}\right\|^{2}\right]+\frac{c}{1-c}\left\|y_{n}-x_{n}\right\|^{2}\right\} \\
\leq & \left(1-\epsilon_{n} \tau\right)\left\{( 1 - \beta _ { n } ) \left[\left\|x_{n}-u_{n}\right\|^{2}+\left(1-\lambda_{n}^{2}\left(\alpha_{n}+\|A\|^{2}\right)^{2}\right)\left\|y_{n, N}-\tilde{y}_{n, N}\right\|^{2}\right.\right. \\
& \left.\left.+v_{2}\left(2 \zeta_{2}-v_{2}\right)\left\|F_{2} t_{n}-F_{2} p\right\|^{2}+v_{1}\left(2 \zeta_{1}-v_{1}\right)\left\|F_{1} \tilde{t}_{n}-F_{1} \tilde{p}\right\|^{2}\right]+\frac{\beta_{n}}{1-\beta_{n}}\left\|y_{n}-x_{n}\right\|^{2}\right\} \\
\leq & \left\|x_{n}-p\right\|^{2}-\left\|x_{n+1}-p\right\|^{2}+2 \lambda_{n} \alpha_{n}\|p\|\left\|p-\tilde{y}_{n, N}\right\|+\epsilon_{n} \frac{1}{\tau}\left\|\gamma\left(\delta_{n} V x_{n}+\left(1-\delta_{n}\right) S x_{n}\right)-\mu F p\right\|^{2} \\
\leq & \left\|x_{n}-x_{n+1}\right\|\left(\left\|x_{n}-p\right\|+\left\|x_{n+1}-p\right\|\right)+2 b \alpha_{n}\|p\|\left\|p-\tilde{y}_{n, N}\right\| \\
& +\epsilon_{n} \frac{1}{\tau}\left\|\gamma\left(\delta_{n} V x_{n}+\left(1-\delta_{n}\right) S x_{n}\right)-\mu F p\right\|^{2} .
\end{aligned}
$$

By Proposition 3.6 we know that the sequences $\left\{x_{n}\right\}$ and $\left\{\tilde{y}_{n, N}\right\}$ are bounded. Therefore, from the asymptotical regularity of $\left\{x_{n}\right\}, \alpha_{n} \rightarrow 0$ and $\epsilon_{n} \rightarrow 0$ we obtain that

$\lim _{n \rightarrow \infty}\left\|x_{n}-u_{n}\right\|=\lim _{n \rightarrow \infty}\left\|F_{2} t_{n}-F_{2} p\right\|=\lim _{n \rightarrow \infty}\left\|F_{1} \tilde{t}_{n}-F_{1} \tilde{p}\right\|=\lim _{n \rightarrow \infty}\left\|y_{n, N}-\tilde{y}_{n, N}\right\|=\lim _{n \rightarrow \infty}\left\|x_{n}-y_{n}\right\|=0$.

Remark 3.1. By the last proposition we have $\omega_{w}\left(x_{n}\right)=\omega_{w}\left(u_{n}\right)$ and $\omega_{s}\left(x_{n}\right)=\omega_{s}\left(u_{n}\right)$, i.e., the sets of strong/weak cluster points of $\left\{x_{n}\right\}$ and $\left\{u_{n}\right\}$ coincide.

Of course, if $\beta_{n, i} \rightarrow \beta_{i} \neq 0$ as $n \rightarrow \infty$, for all indices $i$, the assumptions of Proposition 3.7 are enough to assure that

$$
\lim _{n \rightarrow \infty} \frac{\left\|x_{n+1}-x_{n}\right\|}{\beta_{n, i}}=0, \quad \forall i \in\{1, \ldots, N\} .
$$

In the next proposition, we estimate the case in which at least one sequence $\left\{\beta_{n, k_{0}}\right\}$ is a null sequence.

Proposition 3.9. Let us suppose that $\Omega \neq \varnothing$. Let us suppose that (H0) holds. Moreover, for an index $k_{0} \in\{1, \ldots, N\}, \lim _{n \rightarrow \infty} \beta_{n, k_{0}}=0$ and the following hold:

(H7) for each index $i \in\{1, \ldots, N\}$,

$$
\begin{aligned}
& \lim _{n \rightarrow \infty} \frac{\left|\beta_{n, i}-\beta_{n-1, i}\right|}{\epsilon_{n} \delta_{n} \beta_{n, k_{0}}}=\lim _{n \rightarrow \infty} \frac{\left|\alpha_{n}-\alpha_{n-1}\right|}{\epsilon_{n} \delta_{n} \beta_{n, k_{0}}}=\lim _{n \rightarrow \infty} \frac{\left|\beta_{n}-\beta_{n-1}\right|}{\epsilon_{n} \delta_{n} \beta_{n, k_{0}}}=\lim _{n \rightarrow \infty} \frac{\left|r_{n}-r_{n-1}\right|}{\epsilon_{n} \delta_{n} \beta_{n, k_{0}}}=\lim _{n \rightarrow \infty} \frac{\left|\delta_{n}-\delta_{n-1}\right|}{\delta_{n} \beta_{n, k_{0}}} \\
& =\lim _{n \rightarrow \infty} \frac{\left|\epsilon_{n}-\epsilon_{n-1}\right|}{\epsilon_{n} \delta_{n} \beta_{n, k_{0}}}=\lim _{n \rightarrow \infty} \frac{1}{\epsilon_{n} \delta_{n} \beta_{n, k_{0}}}\left|\frac{\gamma_{n}}{1-\beta_{n}}-\frac{\gamma_{n-1}}{1-\beta_{n-1}}\right|=\lim _{n \rightarrow \infty} \frac{\left|\lambda_{n}-\lambda_{n-1}\right|}{\epsilon_{n} \delta_{n} \beta_{n, k_{0}}}=0 ;
\end{aligned}
$$

(H8) there exists a constant $\delta>0$ such that $\frac{1}{\epsilon_{n} \delta_{n}}\left|\frac{1}{\beta_{n, k_{0}}}-\frac{1}{\beta_{n-1, k_{0}}}\right|<\delta$ for all $n \geq 1$.

If $\left\|u_{n}-u_{n-1}\right\|=o\left(\epsilon_{n} \delta_{n} \beta_{n, k_{0}}\right)$, then

$$
\lim _{n \rightarrow \infty} \frac{\left\|x_{n+1}-x_{n}\right\|}{\beta_{n, k_{0}}}=0 .
$$


Proof. It is clear from (3.25) that

$$
\begin{aligned}
& \frac{\left\|\tilde{y}_{n, N}-\tilde{y}_{n-1, N}\right\|}{\epsilon_{n} \delta_{n} \beta_{n, k_{0}}} \\
& \leq \frac{\left\|y_{n, N}-y_{n-1, N}\right\|}{\epsilon_{n} \delta_{n} \beta_{n, k_{0}}}+\left(\alpha_{n} \frac{\left|\lambda_{n}-\lambda_{n-1}\right|}{\epsilon_{n} \delta_{n} \beta_{n, k_{0}}}+\lambda_{n-1} \frac{\left|\alpha_{n}-\alpha_{n-1}\right|}{\epsilon_{n} \delta_{n} \beta_{n, k_{0}}}\right)\left\|y_{n-1, N}\right\|+\frac{\left|\lambda_{n}-\lambda_{n-1}\right|}{\epsilon_{n} \delta_{n} \beta_{n, k_{0}}}\left\|\nabla f\left(y_{n-1, N}\right)\right\| \\
& \leq \frac{\left\|u_{n}-u_{n-1}\right\|}{\epsilon_{n} \delta_{n} \beta_{n, k_{0}}}+\sum_{k=2}^{N}\left\|S_{k} u_{n-1}-y_{n-1, k-1}\right\| \frac{\left|\beta_{n, k}-\beta_{n-1, k}\right|}{\epsilon_{n} \delta_{n} \beta_{n, k_{0}}}+\left\|S_{1} u_{n-1}-u_{n-1}\right\| \frac{\left|\beta_{n, 1}-\beta_{n-1,1}\right|}{\epsilon_{n} \delta_{n} \beta_{n, k_{0}}} \\
& \quad+\left(\alpha_{n} \frac{\left|\lambda_{n}-\lambda_{n-1}\right|}{\epsilon_{n} \delta_{n} \beta_{n, k_{0}}}+\lambda_{n-1} \frac{\left|\alpha_{n}-\alpha_{n-1}\right|}{\epsilon_{n} \delta_{n} \beta_{n, k_{0}}}\right)\left\|y_{n-1, N}\right\|+\frac{\left|\lambda_{n}-\lambda_{n-1}\right|}{\epsilon_{n} \delta_{n} \beta_{n, k_{0}}}\left\|\nabla f\left(y_{n-1, N}\right)\right\| .
\end{aligned}
$$

According to (H7) and $\left\|u_{n}-u_{n-1}\right\|=o\left(\epsilon_{n} \delta_{n} \beta_{n, k_{0}}\right)$, we get

$$
\lim _{n \rightarrow \infty} \frac{\left\|\tilde{y}_{n, N}-\tilde{y}_{n-1, N}\right\|}{\epsilon_{n} \delta_{n} \beta_{n, k_{0}}}=0 .
$$

Consider (3.29). Dividing both the terms by $\beta_{n, k_{0}}$ we have

$$
\begin{aligned}
\frac{\left\|x_{n+1}-x_{n}\right\|}{\beta_{n, k_{0}}} \leq & \left(1-\epsilon_{n} \delta_{n}(1-\rho) \gamma\right) \frac{\left\|x_{n}-x_{n-1}\right\|}{\beta_{n, k_{0}}}+\widetilde{M}_{1}\left[\frac{\left|r_{n}-r_{n-1}\right|}{\bar{r} \beta_{n, k_{0}}}+\sum_{k=1}^{N} \frac{\left|\beta_{n, k}-\beta_{n-1, k}\right|}{\beta_{n, k_{0}}}\right. \\
& +\frac{\left|\lambda_{n}-\lambda_{n-1}\right|}{\beta_{n, k_{0}}}+\frac{\left|\alpha_{n}-\alpha_{n-1}\right|}{\beta_{n, k_{0}}}+\frac{\left|\beta_{n}-\beta_{n-1}\right|}{\beta_{n, k_{0}}}+\frac{1}{\beta_{n, k_{0}}}\left|\frac{\gamma_{n}}{1-\beta_{n}}-\frac{\gamma_{n-1}}{1-\beta_{n-1}}\right| \\
& \left.+\frac{\left|\epsilon_{n}-\epsilon_{n-1}\right|}{\beta_{n, k_{0}}}+\frac{\epsilon_{n}\left|\delta_{n}-\delta_{n-1}\right|}{\beta_{n, k_{0}}}+\frac{\left\|\tilde{y}_{n, N}-\tilde{y}_{n-1, N}\right\|}{\beta_{n, k_{0}}}\right] .
\end{aligned}
$$

So, by (H8) we have

$$
\begin{aligned}
& \frac{\left\|x_{n+1}-x_{n}\right\|}{\beta_{n, k_{0}}} \leq\left(1-\epsilon_{n} \delta_{n}(1-\rho) \gamma\right) \frac{\left\|x_{n}-x_{n-1}\right\|}{\beta_{n-1, k_{0}}}+\left(1-\epsilon_{n} \delta_{n}(1-\rho) \gamma\right)\left\|x_{n}-x_{n-1}\right\|\left|\frac{1}{\beta_{n, k_{0}}}-\frac{1}{\beta_{n-1, k_{0}}}\right| \\
& +\widetilde{M}_{1}\left[\frac{\left|r_{n}-r_{n-1}\right|}{\bar{r} \beta_{n, k_{0}}}+\sum_{k=1}^{N} \frac{\left|\beta_{n, k}-\beta_{n-1, k}\right|}{\beta_{n, k_{0}}}+\frac{\left|\lambda_{n}-\lambda_{n-1}\right|}{\beta_{n, k_{0}}}+\frac{\left|\alpha_{n}-\alpha_{n-1}\right|}{\beta_{n, k_{0}}}+\frac{\left|\beta_{n}-\beta_{n-1}\right|}{\beta_{n, k_{0}}}\right. \\
& \left.+\frac{1}{\beta_{n, k_{0}}}\left|\frac{\gamma_{n}}{1-\beta_{n}}-\frac{\gamma_{n-1}}{1-\beta_{n-1}}\right|+\frac{\left|\epsilon_{n}-\epsilon_{n-1}\right|}{\beta_{n, k_{0}}}+\frac{\epsilon_{n}\left|\delta_{n}-\delta_{n-1}\right|}{\beta_{n, k_{0}}}+\frac{\left\|\tilde{y}_{n, N}-\tilde{y}_{n-1, N}\right\|}{\beta_{n, k_{0}}}\right] \\
& \leq\left(1-\epsilon_{n} \delta_{n}(1-\rho) \gamma\right) \frac{\left\|x_{n}-x_{n-1}\right\|}{\beta_{n-1, k_{0}}}+\left\|x_{n}-x_{n-1}\right\|\left|\frac{1}{\beta_{n, k_{0}}}-\frac{1}{\beta_{n-1, k_{0}}}\right| \\
& +\widetilde{M}_{1}\left[\frac{\left|r_{n}-r_{n-1}\right|}{\bar{r} \beta_{n, k_{0}}}+\sum_{k=1}^{N} \frac{\left|\beta_{n, k}-\beta_{n-1, k}\right|}{\beta_{n, k_{0}}}+\frac{\left|\lambda_{n}-\lambda_{n-1}\right|}{\beta_{n, k_{0}}}+\frac{\left|\alpha_{n}-\alpha_{n-1}\right|}{\beta_{n, k_{0}}}+\frac{\left|\beta_{n}-\beta_{n-1}\right|}{\beta_{n, k_{0}}}\right. \\
& \left.+\frac{1}{\beta_{n, k_{0}}}\left|\frac{\gamma_{n}}{1-\beta_{n}}-\frac{\gamma_{n-1}}{1-\beta_{n-1}}\right|+\frac{\left|\epsilon_{n}-\epsilon_{n-1}\right|}{\beta_{n, k_{0}}}+\frac{\epsilon_{n}\left|\delta_{n}-\delta_{n-1}\right|}{\beta_{n, k_{0}}}+\frac{\left\|\tilde{y}_{n, N}-\tilde{y}_{n-1, N}\right\|}{\beta_{n, k_{0}}}\right] \\
& \leq\left(1-\epsilon_{n} \delta_{n}(1-\rho) \gamma\right) \frac{\left\|x_{n}-x_{n-1}\right\|}{\beta_{n-1, k_{0}}}+\epsilon_{n} \delta_{n} \delta\left\|x_{n}-x_{n-1}\right\| \\
& +\widetilde{M}_{1}\left[\frac{\left|r_{n}-r_{n-1}\right|}{\bar{r} \beta_{n, k_{0}}}+\sum_{k=1}^{N} \frac{\left|\beta_{n, k}-\beta_{n-1, k}\right|}{\beta_{n, k_{0}}}+\frac{\left|\lambda_{n}-\lambda_{n-1}\right|}{\beta_{n, k_{0}}}+\frac{\left|\alpha_{n}-\alpha_{n-1}\right|}{\beta_{n, k_{0}}}+\frac{\left|\beta_{n}-\beta_{n-1}\right|}{\beta_{n, k_{0}}}\right.
\end{aligned}
$$




$$
\begin{aligned}
& \left.+\frac{1}{\beta_{n, k_{0}}}\left|\frac{\gamma_{n}}{1-\beta_{n}}-\frac{\gamma_{n-1}}{1-\beta_{n-1}}\right|+\frac{\left|\epsilon_{n}-\epsilon_{n-1}\right|}{\beta_{n, k_{0}}}+\frac{\epsilon_{n}\left|\delta_{n}-\delta_{n-1}\right|}{\beta_{n, k_{0}}}+\frac{\left\|\tilde{y}_{n, N}-\tilde{y}_{n-1, N}\right\|}{\beta_{n, k_{0}}}\right] \\
= & \left(1-\epsilon_{n} \delta_{n}(1-\rho) \gamma\right) \frac{\left\|x_{n}-x_{n-1}\right\|}{\beta_{n-1, k_{0}}}+\epsilon_{n} \delta_{n}(1-\rho) \gamma \cdot \frac{1}{(1-\rho) \gamma}\left\{\delta\left\|x_{n}-x_{n-1}\right\|\right. \\
& +\widetilde{M}_{1}\left[\frac{\left|r_{n}-r_{n-1}\right|}{\bar{r} \epsilon_{n} \delta_{n} \beta_{n, k_{0}}}+\sum_{k=1}^{N} \frac{\left|\beta_{n, k}-\beta_{n-1, k}\right|}{\epsilon_{n} \delta_{n} \beta_{n, k_{0}}}+\frac{\left|\lambda_{n}-\lambda_{n-1}\right|}{\epsilon_{n} \delta_{n} \beta_{n, k_{0}}}+\frac{\left|\alpha_{n}-\alpha_{n-1}\right|}{\epsilon_{n} \delta_{n} \beta_{n, k_{0}}}+\frac{\left|\beta_{n}-\beta_{n-1}\right|}{\epsilon_{n} \delta_{n} \beta_{n, k_{0}}}\right. \\
& \left.\left.+\frac{1}{\epsilon_{n} \delta_{n} \beta_{n, k_{0}}}\left|\frac{\gamma_{n}}{1-\beta_{n}}-\frac{\gamma_{n-1}}{1-\beta_{n-1}}\right|+\frac{\left|\epsilon_{n}-\epsilon_{n-1}\right|}{\epsilon_{n} \delta_{n} \beta_{n, k_{0}}}+\frac{\left|\delta_{n}-\delta_{n-1}\right|}{\delta_{n} \beta_{n, k_{0}}}+\frac{\left\|\tilde{y}_{n, N}-\tilde{y}_{n-1, N}\right\|}{\epsilon_{n} \delta_{n} \beta_{n, k_{0}}}\right]\right\} .
\end{aligned}
$$

Therefore, utilizing Lemma 2.8, from (3.33), (H0), (H7) and the asymptotical regularity of $\left\{x_{n}\right\}$ (due to Proposition 3.7), we deduce that

$$
\lim _{n \rightarrow \infty} \frac{\left\|x_{n+1}-x_{n}\right\|}{\beta_{n, k_{0}}}=0 .
$$

Proposition 3.10. Let us suppose that $\Omega \neq \varnothing$. Let us suppose that (H0)-(H6) hold. If $\| u_{n}-$ $u_{n-1} \|=o\left(\epsilon_{n} \delta_{n}\right)$, then $\left\|z_{n}-t_{n}\right\| \rightarrow 0$ as $n \rightarrow \infty$.

Proof. Let $p \in \Omega$. In terms of the firm nonexpansivity of $P_{C}$ and the $\zeta_{j}$-inverse strong monotonicity of $F_{j}$ for $j=1,2$, we obtain from $v_{j} \in\left(0,2 \zeta_{j}\right), j=1,2$ and (3.10) that

$$
\begin{aligned}
\left\|\tilde{t}_{n}-\tilde{p}\right\|^{2}= & \left\|P_{C}\left(I-v_{2} F_{2}\right) t_{n}-P_{C}\left(I-v_{2} F_{2}\right) p\right\|^{2} \\
\leq & \left\langle\left(I-v_{2} F_{2}\right) t_{n}-\left(I-v_{2} F_{2}\right) p, \tilde{t}_{n}-\tilde{p}\right\rangle \\
= & \frac{1}{2}\left[\left\|\left(I-v_{2} F_{2}\right) t_{n}-\left(I-v_{2} F_{2}\right) p\right\|^{2}+\left\|\tilde{t}_{n}-\tilde{p}\right\|^{2}\right. \\
& \left.-\left\|\left(I-v_{2} F_{2}\right) t_{n}-\left(I-v_{2} F_{2}\right) p-\left(\tilde{t}_{n}-\tilde{p}\right)\right\|^{2}\right] \\
\leq & \frac{1}{2}\left[\left\|t_{n}-p\right\|^{2}+\left\|\tilde{t}_{n}-\tilde{p}\right\|^{2}-\left\|\left(t_{n}-\tilde{t}_{n}\right)-v_{2}\left(F_{2} t_{n}-F_{2} p\right)-(p-\tilde{p})\right\|^{2}\right] \\
= & \frac{1}{2}\left[\left\|t_{n}-p\right\|^{2}+\left\|\tilde{t}_{n}-\tilde{p}\right\|^{2}-\left\|\left(t_{n}-\tilde{t}_{n}\right)-(p-\tilde{p})\right\|^{2}\right. \\
& \left.+2 v_{2}\left\langle\left(t_{n}-\tilde{t}_{n}\right)-(p-\tilde{p}), F_{2} t_{n}-F_{2} p\right\rangle-v_{2}^{2}\left\|F_{2} t_{n}-F_{2} p\right\|^{2}\right],
\end{aligned}
$$

and

$$
\begin{aligned}
\left\|z_{n}-p\right\|^{2}= & \left\|P_{C}\left(I-v_{1} F_{1}\right) \tilde{t}_{n}-P_{C}\left(I-v_{1} F_{1}\right) \tilde{p}\right\|^{2} \\
\leq & \left\langle\left(I-v_{1} F_{1}\right) \tilde{t}_{n}-\left(I-v_{1} F_{1}\right) \tilde{p}, z_{n}-p\right\rangle \\
= & \frac{1}{2}\left[\left\|\left(I-v_{1} F_{1}\right) \tilde{t}_{n}-\left(I-v_{1} F_{1}\right) \tilde{p}\right\|^{2}+\left\|z_{n}-p\right\|^{2}\right. \\
& \left.-\left\|\left(I-v_{1} F_{1}\right) \tilde{t}_{n}-\left(I-v_{1} F_{1}\right) \tilde{p}-\left(z_{n}-p\right)\right\|^{2}\right] \\
\leq & \frac{1}{2}\left[\left\|\tilde{t}_{n}-\tilde{p}\right\|^{2}+\left\|z_{n}-p\right\|^{2}-\left\|\left(\tilde{t}_{n}-z_{n}\right)+(p-\tilde{p})\right\|^{2}\right. \\
& \left.+2 v_{1}\left\langle F_{1} \tilde{t}_{n}-F_{1} \tilde{p},\left(\tilde{t}_{n}-z_{n}\right)+(p-\tilde{p})\right\rangle-v_{1}^{2}\left\|F_{1} \tilde{t}_{n}-F_{1} \tilde{p}\right\|^{2}\right] \\
\leq & \frac{1}{2}\left[\left\|t_{n}-p\right\|^{2}+\left\|z_{n}-p\right\|^{2}-\left\|\left(\tilde{t}_{n}-z_{n}\right)+(p-\tilde{p})\right\|^{2}\right.
\end{aligned}
$$




$$
\left.+2 v_{1}\left\langle F_{1} \tilde{t}_{n}-F_{1} \tilde{p},\left(\tilde{t}_{n}-z_{n}\right)+(p-\tilde{p})\right\rangle\right]
$$

Thus, we have

$$
\begin{aligned}
\left\|\tilde{t}_{n}-\tilde{p}\right\|^{2} \leq & \left\|t_{n}-p\right\|^{2}-\left\|\left(t_{n}-\tilde{t}_{n}\right)-(p-\tilde{p})\right\|^{2} \\
& +2 v_{2}\left\langle\left(t_{n}-\tilde{t}_{n}\right)-(p-\tilde{p}), F_{2} t_{n}-F_{2} p\right\rangle-v_{2}^{2}\left\|F_{2} t_{n}-F_{2} p\right\|^{2},
\end{aligned}
$$

and

$$
\left\|z_{n}-p\right\|^{2} \leq\left\|t_{n}-p\right\|^{2}-\left\|\left(\tilde{t}_{n}-z_{n}\right)+(p-\tilde{p})\right\|^{2}+2 v_{1}\left\|F_{1} \tilde{t}_{n}-F_{1} \tilde{p}\right\|\left\|\left(\tilde{t}_{n}-z_{n}\right)+(p-\tilde{p})\right\| .
$$

Consequently, from (3.10), (3.15), (3.31) and (3.34), it follows that

$$
\begin{aligned}
\left\|y_{n}-p\right\|^{2} \leq & \beta_{n}\left\|x_{n}-p\right\|^{2}+\left(1-\beta_{n}\right)\left\|z_{n}-p\right\|^{2} \\
\leq & \beta_{n}\left\|x_{n}-p\right\|^{2}+\left(1-\beta_{n}\right)\left\|\tilde{t}_{n}-\tilde{p}\right\|^{2} \\
\leq & \beta_{n}\left\|x_{n}-p\right\|^{2}+\left(1-\beta_{n}\right)\left[\left\|t_{n}-p\right\|^{2}-\left\|\left(t_{n}-\tilde{t}_{n}\right)-(p-\tilde{p})\right\|^{2}\right. \\
& \left.+2 v_{2}\left\|\left(t_{n}-\tilde{t}_{n}\right)-(p-\tilde{p})\right\|\left\|F_{2} t_{n}-F_{2} p\right\|\right] \\
\leq & \beta_{n}\left\|x_{n}-p\right\|^{2}+\left(1-\beta_{n}\right)\left[\left(\left\|x_{n}-p\right\|+\sqrt{2} \lambda_{n} \alpha_{n}\|p\|\right)^{2}\right. \\
& \left.-\left\|\left(t_{n}-\tilde{t}_{n}\right)-(p-\tilde{p})\right\|^{2}+2 v_{2}\left\|\left(t_{n}-\tilde{t}_{n}\right)-(p-\tilde{p})\right\|\left\|F_{2} t_{n}-F_{2} p\right\|\right] \\
\leq & \left(\left\|x_{n}-p\right\|+\sqrt{2} \lambda_{n} \alpha_{n}\|p\|\right)^{2}-\left(1-\beta_{n}\right)\left\|\left(t_{n}-\tilde{t}_{n}\right)-(p-\tilde{p})\right\|^{2} \\
& +2 v_{2}\left\|\left(t_{n}-\tilde{t}_{n}\right)-(p-\tilde{p})\right\|\left\|F_{2} t_{n}-F_{2} p\right\|,
\end{aligned}
$$

which yields

$$
\begin{aligned}
&(1-d)\left\|\left(t_{n}-\tilde{t}_{n}\right)-(p-\tilde{p})\right\|^{2} \\
& \leq\left(1-\beta_{n}\right)\left\|\left(t_{n}-\tilde{t}_{n}\right)-(p-\tilde{p})\right\|^{2} \\
& \leq\left(\left\|x_{n}-p\right\|+\sqrt{2} \lambda_{n} \alpha_{n}\|p\|\right)^{2}-\left\|y_{n}-p\right\|^{2}+2 v_{2}\left\|\left(t_{n}-\tilde{t}_{n}\right)-(p-\tilde{p})\right\|\left\|F_{2} t_{n}-F_{2} p\right\| \\
& \leq\left(\left\|x_{n}-y_{n}\right\|+\sqrt{2} \lambda_{n} \alpha_{n}\|p\|\right)\left(\left\|x_{n}-p\right\|+\left\|y_{n}-p\right\|+\sqrt{2} \lambda_{n} \alpha_{n}\|p\|\right) \\
&+2 v_{2}\left\|\left(t_{n}-\tilde{t}_{n}\right)-(p-\tilde{p})\right\|\left\|F_{2} t_{n}-F_{2} p\right\| .
\end{aligned}
$$

Since $\lim _{n \rightarrow \infty} \alpha_{n}=0$ and $\left\{x_{n}\right\},\left\{y_{n}\right\},\left\{t_{n}\right\}$ and $\left\{\tilde{t}_{n}\right\}$ are bounded, we deduce from (3.32) that

$$
\lim _{n \rightarrow \infty}\left\|\left(t_{n}-\tilde{t}_{n}\right)-(p-\tilde{p})\right\|=0 .
$$

Furthermore, from (3.15), (3.31) and (3.35), it follows that

$$
\begin{aligned}
\left\|y_{n}-p\right\|^{2} \leq & \beta_{n}\left\|x_{n}-p\right\|^{2}+\left(1-\beta_{n}\right)\left\|z_{n}-p\right\|^{2} \\
\leq & \beta_{n}\left\|x_{n}-p\right\|^{2}+\left(1-\beta_{n}\right)\left[\left\|t_{n}-p\right\|^{2}-\left\|\left(\tilde{t}_{n}-z_{n}\right)+(p-\tilde{p})\right\|^{2}\right. \\
& \left.+2 v_{1}\left\|F_{1} \tilde{t}_{n}-F_{1} \tilde{p}\right\|\left\|\left(\tilde{t}_{n}-z_{n}\right)+(p-\tilde{p})\right\|\right] \\
\leq & \beta_{n}\left\|x_{n}-p\right\|^{2}+\left(1-\beta_{n}\right)\left[\left(\left\|x_{n}-p\right\|+\sqrt{2} \lambda_{n} \alpha_{n}\|p\|\right)^{2}-\left\|\left(\tilde{t}_{n}-z_{n}\right)+(p-\tilde{p})\right\|^{2}\right.
\end{aligned}
$$




$$
\begin{aligned}
& \left.+2 v_{1}\left\|F_{1} \tilde{t}_{n}-F_{1} \tilde{p}\right\|\left\|\left(\tilde{t}_{n}-z_{n}\right)+(p-\tilde{p})\right\|\right] \\
\leq & \left(\left\|x_{n}-p\right\|+\sqrt{2} \lambda_{n} \alpha \alpha_{n}\|p\|\right)^{2}-\left(1-\beta_{n}\right)\left\|\left(\tilde{t}_{n}-z_{n}\right)+(p-\tilde{p})\right\|^{2} \\
& +2 v_{1}\left\|F_{1} \tilde{t}_{n}-F_{1} \tilde{p}\right\|\left\|\left(\tilde{t}_{n}-z_{n}\right)+(p-\tilde{p})\right\|,
\end{aligned}
$$

which leads to

$$
\begin{aligned}
&(1-d)\left\|\left(\tilde{t}_{n}-z_{n}\right)+(p-\tilde{p})\right\|^{2} \\
& \leq\left(1-\beta_{n}\right)\left\|\left(\tilde{t}_{n}-z_{n}\right)+(p-\tilde{p})\right\|^{2} \\
& \leq\left(\left\|x_{n}-p\right\|+\sqrt{2} \lambda_{n} \alpha_{n}\|p\|\right)^{2}-\left\|y_{n}-p\right\|^{2}+2 v_{1}\left\|F_{1} \tilde{t}_{n}-F_{1} \tilde{p}\right\|\left\|\left(\tilde{t}_{n}-z_{n}\right)+(p-\tilde{p})\right\| \\
& \leq\left(\left\|x_{n}-y_{n}\right\|+\sqrt{2} \lambda_{n} \alpha_{n}\|p\|\right)\left(\left\|x_{n}-p\right\|+\left\|y_{n}-p\right\|+\sqrt{2} \lambda_{n} \alpha_{n}\|p\|\right) \\
& \quad+2 v_{1}\left\|F_{1} \tilde{t}_{n}-F_{1} \tilde{p}\right\|\left\|\left(\tilde{t}_{n}-z_{n}\right)+(p-\tilde{p})\right\| .
\end{aligned}
$$

Since $\lim _{n \rightarrow \infty} \alpha_{n}=0$ and $\left\{x_{n}\right\},\left\{y_{n}\right\},\left\{z_{n}\right\}$ and $\left\{\tilde{t}_{n}\right\}$ are bounded, we deduce from (3.32) that

$$
\lim _{n \rightarrow \infty}\left\|\left(\tilde{t}_{n}-z_{n}\right)+(p-\tilde{p})\right\|=0 .
$$

Note that

$$
\left\|t_{n}-z_{n}\right\| \leq\left\|\left(t_{n}-\tilde{t}_{n}\right)-(p-\tilde{p})\right\|+\left\|\left(\tilde{t}_{n}-z_{n}\right)+(p-\tilde{p})\right\| .
$$

Hence from (3.36) and (3.37) we get

$$
\lim _{n \rightarrow \infty}\left\|t_{n}-z_{n}\right\|=\lim _{n \rightarrow \infty}\left\|t_{n}-G t_{n}\right\|=0
$$

Proposition 3.11. Let us suppose that $\Omega \neq \varnothing$. Let us suppose that

$$
0<\liminf _{n \rightarrow \infty} \beta_{n, i} \leq \limsup _{n \rightarrow \infty} \beta_{n, i}<1
$$

for each $i=1, \ldots, N$. Moreover, suppose that $\left\|u_{n}-u_{n-1}\right\|=o\left(\epsilon_{n} \delta_{n}\right)$ and $(\mathrm{H} 0)-(\mathrm{H} 6)$ are satisfied. Then, $\lim _{n \rightarrow \infty}\left\|S_{i} u_{n}-u_{n}\right\|=0$ for each $i=1, \ldots, N$ as $n \rightarrow \infty$.

Proof. First of all, it is clear that

$$
\begin{aligned}
\left\|t_{n}-\tilde{y}_{n, N}\right\| & =\left\|P_{C}\left(y_{n, N}-\lambda_{n} \nabla f_{\alpha_{n}}\left(\tilde{y}_{n, N}\right)\right)-P_{C}\left(y_{n, N}-\lambda_{n} \nabla f_{\alpha_{n}}\left(y_{n, N}\right)\right)\right\| \\
& \leq\left\|\left(y_{n, N}-\lambda_{n} \nabla f_{\alpha_{n}}\left(\tilde{y}_{n, N}\right)\right)-\left(y_{n, N}-\lambda_{n} \nabla f_{\alpha_{n}}\left(y_{n, N}\right)\right)\right\| \\
& =\lambda_{n}\left\|\nabla f_{\alpha_{n}}\left(\tilde{y}_{n, N}\right)-\nabla f_{\alpha_{n}}\left(y_{n, N}\right)\right\| \\
& \leq \lambda_{n}\left(\alpha_{n}+\|A\|^{2}\right)\left\|\tilde{y}_{n, N}-y_{n, N}\right\| \\
& \leq\left\|\tilde{y}_{n, N}-y_{n, N}\right\| .
\end{aligned}
$$

By Proposition 3.8, we get

$$
\lim _{n \rightarrow \infty}\left\|t_{n}-\tilde{y}_{n, N}\right\|=0,
$$


which together with (3.31), implies that

$$
\lim _{n \rightarrow \infty}\left\|t_{n}-y_{n, N}\right\|=0
$$

Let us show that for each $i \in\{1, \ldots, N\}$, one has $\left\|S_{i} u_{n}-y_{n, i-1}\right\| \rightarrow 0$ as $n \rightarrow \infty$. Let $p \in \Omega$. When $i=N$, by Lemma 2.2 (b) we have from (3.9), (3.10), (3.15) and (3.31)

$$
\begin{aligned}
\left\|y_{n}-p\right\|^{2} \leq & \beta_{n}\left\|x_{n}-p\right\|^{2}+\left(1-\beta_{n}\right)\left\|z_{n}-p\right\|^{2} \\
\leq & \beta_{n}\left\|x_{n}-p\right\|^{2}+\left(1-\beta_{n}\right)\left\|t_{n}-p\right\|^{2} \\
\leq & \beta_{n}\left\|x_{n}-p\right\|^{2}+\left(1-\beta_{n}\right)\left[\left\|y_{n, N}-p\right\|^{2}+2 \lambda_{n} \alpha_{n}\|p\|\left\|\tilde{y}_{n, N}-p\right\|\right] \\
= & \beta_{n}\left\|x_{n}-p\right\|^{2}+\left(1-\beta_{n}\right)\left[\beta_{n, N}\left\|S_{N} u_{n}-p\right\|^{2}+\left(1-\beta_{n, N}\right)\left\|y_{n, N-1}-p\right\|^{2}\right. \\
& \left.-\beta_{n, N}\left(1-\beta_{n, N}\right)\left\|S_{N} u_{n}-y_{n, N-1}\right\|^{2}+2 \lambda_{n} \alpha_{n}\|p\|\left\|\tilde{y}_{n, N}-p\right\|\right] \\
\leq & \beta_{n}\left\|x_{n}-p\right\|^{2}+\left(1-\beta_{n}\right)\left[\beta_{n, N}\left\|u_{n}-p\right\|^{2}+\left(1-\beta_{n, N}\right)\left\|u_{n}-p\right\|^{2}\right. \\
& \left.-\beta_{n, N}\left(1-\beta_{n, N}\right)\left\|S_{N} u_{n}-y_{n, N-1}\right\|^{2}+2 \lambda_{n} \alpha_{n}\|p\|\left\|\tilde{y}_{n, N}-p\right\|\right] \\
= & \beta_{n}\left\|x_{n}-p\right\|^{2}+\left(1-\beta_{n}\right)\left[\left\|u_{n}-p\right\|^{2}-\beta_{n, N}\left(1-\beta_{n, N}\right)\left\|S_{N} u_{n}-y_{n, N-1}\right\|^{2}\right. \\
& \left.+2 \lambda_{n} \alpha_{n}\|p\|\left\|\tilde{y}_{n, N}-p\right\|\right] \\
\leq & \beta_{n}\left\|x_{n}-p\right\|^{2}+\left(1-\beta_{n}\right)\left[\left\|x_{n}-p\right\|^{2}-\beta_{n, N}\left(1-\beta_{n, N}\right)\left\|S_{N} u_{n}-y_{n, N-1}\right\|^{2}\right. \\
& \left.+2 \lambda_{n} \alpha_{n}\|p\|\left\|\tilde{y}_{n, N}-p\right\|\right] \\
\leq & \left\|x_{n}-p\right\|^{2}-\left(1-\beta_{n}\right) \beta_{n, N}\left(1-\beta_{n, N}\right)\left\|S_{N} u_{n}-y_{n, N-1}\right\|^{2}+2 \lambda_{n} \alpha_{n}\|p\|\left\|\tilde{y}_{n, N}-p\right\|,
\end{aligned}
$$

which together with $\left\{\lambda_{n}\right\} \subset[a, b] \subset\left(0, \frac{1}{\|A\|^{2}}\right)$ and $\left\{\beta_{n}\right\} \subset[c, d] \subset(0,1)$, leads to

$$
\begin{aligned}
& (1-d) \beta_{n, N}\left(1-\beta_{n, N}\right)\left\|S_{N} u_{n}-y_{n, N-1}\right\|^{2} \\
& \leq\left(1-\beta_{n}\right) \beta_{n, N}\left(1-\beta_{n, N}\right)\left\|S_{N} u_{n}-y_{n, N-1}\right\|^{2} \\
& \leq\left\|x_{n}-p\right\|^{2}-\left\|y_{n}-p\right\|^{2}+2 \lambda_{n} \alpha_{n}\|p\|\left\|\tilde{y}_{n, N}-p\right\| \\
& \leq\left\|x_{n}-y_{n}\right\|\left(\left\|x_{n}-p\right\|+\left\|y_{n}-p\right\|\right)+2 b \alpha_{n}\|p\|\left\|\tilde{y}_{n, N}-p\right\| .
\end{aligned}
$$

Since $\alpha_{n} \rightarrow 0,0<\liminf _{n \rightarrow \infty} \beta_{n, N} \leq \limsup _{n \rightarrow \infty} \beta_{n, N}<1$ and $\lim _{n \rightarrow \infty}\left\|x_{n}-y_{n}\right\|=0$ (due to (3.32)), it is known that $\left\{\left\|S_{N} u_{n}-y_{n, N-1}\right\|\right\}$ is a null sequence.

Let $i \in\{1, \ldots, N-1\}$. Then one has

$$
\begin{aligned}
\left\|y_{n}-p\right\|^{2} \leq & \beta_{n}\left\|x_{n}-p\right\|^{2}+\left(1-\beta_{n}\right)\left\|t_{n}-p\right\|^{2} \\
\leq & \beta_{n}\left\|x_{n}-p\right\|^{2}+\left(1-\beta_{n}\right)\left[\left\|y_{n, N}-p\right\|^{2}+2 \lambda_{n} \alpha_{n}\|p\|\left\|\tilde{y}_{n, N}-p\right\|\right] \\
\leq & \beta_{n}\left\|x_{n}-p\right\|^{2}+\left(1-\beta_{n}\right)\left[\beta_{n, N}\left\|S_{N} u_{n}-p\right\|^{2}+\left(1-\beta_{n, N}\right)\left\|y_{n, N-1}-p\right\|^{2}\right. \\
& \left.+2 \lambda_{n} \alpha_{n}\|p\|\left\|\tilde{y}_{n, N}-p\right\|\right] \\
\leq & \beta_{n}\left\|x_{n}-p\right\|^{2}+\left(1-\beta_{n}\right)\left[\beta_{n, N}\left\|x_{n}-p\right\|^{2}+\left(1-\beta_{n, N}\right)\left\|y_{n, N-1}-p\right\|^{2}\right.
\end{aligned}
$$




$$
\begin{aligned}
& \left.+2 \lambda_{n} \alpha_{n}\|p\|\left\|\tilde{y}_{n, N}-p\right\|\right] \\
\leq & \beta_{n}\left\|x_{n}-p\right\|^{2}+\left(1-\beta_{n}\right)\left\{\beta_{n, N}\left\|x_{n}-p\right\|^{2}+\left(1-\beta_{n, N}\right)\left[\beta_{n, N-1}\left\|S_{N-1} u_{n}-p\right\|^{2}\right.\right. \\
& \left.\left.+\left(1-\beta_{n, N-1}\right)\left\|y_{n, N-2}-p\right\|^{2}\right]+2 \lambda_{n} \alpha_{n}\|p\|\left\|\tilde{y}_{n, N}-p\right\|\right\} \\
\leq & \beta_{n}\left\|x_{n}-p\right\|^{2}+\left(1-\beta_{n}\right)\left\{\left(\beta_{n, N}+\left(1-\beta_{n, N}\right) \beta_{n, N-1}\right)\left\|x_{n}-p\right\|^{2}\right. \\
& \left.+\prod_{k=N-1}^{N}\left(1-\beta_{n, k}\right)\left\|y_{n, N-2}-p\right\|^{2}+2 \lambda_{n} \alpha_{n}\|p\|\left\|\tilde{y}_{n, N}-p\right\|\right\},
\end{aligned}
$$

and so, after $(N-i+1)$-iterations,

$$
\begin{aligned}
\left\|y_{n}-p\right\|^{2} \leq & \beta_{n}\left\|x_{n}-p\right\|^{2}+\left(1-\beta_{n}\right)\left\{\left(\beta_{n, N}+\sum_{j=i+2}^{N}\left(\prod_{l=j}^{N}\left(1-\beta_{n, l}\right)\right) \beta_{n, j-1}\right)\left\|x_{n}-p\right\|^{2}\right. \\
& \left.+\prod_{k=i+1}^{N}\left(1-\beta_{n, k}\right)\left\|y_{n, i}-p\right\|^{2}+2 \lambda_{n} \alpha_{n}\|p\|\left\|\tilde{y}_{n, N}-p\right\|\right\} \\
= & \beta_{n}\left\|x_{n}-p\right\|^{2}+\left(1-\beta_{n}\right)\left\{\left(\beta_{n, N}+\sum_{j=i+2}^{N}\left(\prod_{l=j}^{N}\left(1-\beta_{n, l}\right)\right) \beta_{n, j-1}\right)\left\|x_{n}-p\right\|^{2}\right. \\
& +\prod_{k=i+1}^{N}\left(1-\beta_{n, k}\right)\left[\beta_{n, i}\left\|S_{i} u_{n}-p\right\|^{2}+\left(1-\beta_{n, i}\right)\left\|y_{n, i-1}-p\right\|^{2}\right. \\
& \left.\left.-\beta_{n, i}\left(1-\beta_{n, i}\right)\left\|S_{i} u_{n}-y_{n, i-1}\right\|^{2}\right]+2 \lambda_{n} \alpha_{n}\|p\|\left\|\tilde{y}_{n, N}-p\right\|\right\} \\
\leq & \beta_{n}\left\|x_{n}-p\right\|^{2}+\left(1-\beta_{n}\right)\left[\left\|x_{n}-p\right\|^{2}-\beta_{n, i} \prod_{k=i}^{N}\left(1-\beta_{n, k}\right)\left\|S_{i} u_{n}-y_{n, i-1}\right\|^{2}\right. \\
& \left.+2 \lambda_{n} \alpha_{n}\|p\|\left\|\tilde{y}_{n, N}-p\right\|\right] \\
\leq & \left\|x_{n}-p\right\|^{2}-\left(1-\beta_{n}\right) \beta_{n, i} \prod_{k=i}^{N}\left(1-\beta_{n, k}\right)\left\|S_{i} u_{n}-y_{n, i-1}\right\|^{2}+2 \lambda_{n} \alpha_{n}\|p\|\left\|\tilde{y}_{n, N}-p\right\| .
\end{aligned}
$$

Again we obtain that

$$
\begin{aligned}
& (1-d) \beta_{n, i} \prod_{k=i}^{N}\left(1-\beta_{n, k}\right)\left\|S_{i} u_{n}-y_{n, i-1}\right\|^{2} \\
& \leq\left(1-\beta_{n}\right) \beta_{n, i} \prod_{k=i}^{N}\left(1-\beta_{n, k}\right)\left\|S_{i} u_{n}-y_{n, i-1}\right\|^{2} \\
& \leq\left\|x_{n}-p\right\|^{2}-\left\|y_{n}-p\right\|^{2}+2 \lambda_{n} \alpha_{n}\|p\|\left\|\tilde{y}_{n, N}-p\right\| \\
& \leq\left\|x_{n}-y_{n}\right\|\left(\left\|x_{n}-p\right\|+\left\|y_{n}-p\right\|\right)+2 b \alpha_{n}\|p\|\left\|\tilde{y}_{n, N}-p\right\| .
\end{aligned}
$$

Since $\alpha_{n} \rightarrow 0,0<\liminf _{n \rightarrow \infty} \beta_{n, i} \leq \limsup _{n \rightarrow \infty} \beta_{n, i}<1$ for each $i=1, \ldots, N-1$, and $\lim _{n \rightarrow \infty}$ $\left\|x_{n}-y_{n}\right\|=0$ (due to (3.32)), it is known that

$$
\lim _{n \rightarrow \infty}\left\|S_{i} u_{n}-y_{n, i-1}\right\|=0
$$

Obviously for $i=1$, we have $\left\|S_{1} u_{n}-u_{n}\right\| \rightarrow 0$. 
To conclude, we have that

$$
\left\|S_{2} u_{n}-u_{n}\right\| \leq\left\|S_{2} u_{n}-y_{n, 1}\right\|+\left\|y_{n, 1}-u_{n}\right\|=\left\|S_{2} u_{n}-y_{n, 1}\right\|+\beta_{n, 1}\left\|S_{1} u_{n}-u_{n}\right\|
$$

from which $\left\|S_{2} u_{n}-u_{n}\right\| \rightarrow 0$. Thus by induction $\left\|S_{i} u_{n}-u_{n}\right\| \rightarrow 0$ for all $i=2, \ldots, N$ since it is enough to observe that

$$
\begin{aligned}
\left\|S_{i} u_{n}-u_{n}\right\| & \leq\left\|S_{i} u_{n}-y_{n, i-1}\right\|+\left\|y_{n, i-1}-S_{i-1} u_{n}\right\|+\left\|S_{i-1} u_{n}-u_{n}\right\| \\
& \leq\left\|S_{i} u_{n}-y_{n, i-1}\right\|+\left(1-\beta_{n, i-1}\right)\left\|S_{i-1} u_{n}-y_{n, i-2}\right\|+\left\|S_{i-1} u_{n}-u_{n}\right\| .
\end{aligned}
$$

Remark 3.2. As an example, we consider $N=2$ and the sequences:

(a) $\beta_{n}=\frac{1}{2}+\frac{2}{n}, \quad \gamma_{n}=\sigma_{n}=\frac{1}{4}-\frac{1}{n}, \quad \forall n>4$;

(b) $\lambda_{n}=\frac{1}{2\|A\|^{2}}-\frac{1}{2 n}, \quad \forall n>\|A\|^{2}$;

(c) $\alpha_{n}=\frac{1}{n^{2}}, \quad \epsilon_{n}=\frac{1}{n^{3 / 4}}, \quad \delta_{n}=\frac{1}{n^{1 / 4}}, \quad r_{n}=2-\frac{1}{n}, \quad \forall n>1$;

(d) $\beta_{n, 1}=\frac{1}{2}-\frac{1}{n}, \quad \beta_{n, 2}=\frac{1}{2}-\frac{1}{n^{2}}, \quad \forall n>2$.

Then they satisfy the hypotheses on the parameter sequences in Proposition 3.11.

Proposition 3.12. Let us suppose that $\Omega \neq \varnothing$ and $\beta_{n, i} \rightarrow \beta_{i}$ for all $i$ as $n \rightarrow \infty$. Suppose there exists $k \in\{1, \ldots, N\}$ such that $\beta_{n, k} \rightarrow 0$ as $n \rightarrow \infty$. Let $k_{0} \in\{1, \ldots, N\}$ the largest index such that $\beta_{n, k_{0}} \rightarrow 0$ as $n \rightarrow \infty$. Suppose that

(i) $\frac{\alpha_{n}+\epsilon_{n}}{\beta_{n, k_{0}}} \rightarrow 0$ as $n \rightarrow \infty$;

(ii) if $i \leq k_{0}$ and $\beta_{n, i} \rightarrow 0$ then $\frac{\beta_{n, k_{0}}}{\beta_{n, i}} \rightarrow 0$ as $n \rightarrow \infty$;

(iii) if $\beta_{n, i} \rightarrow \beta_{i} \neq 0$ then $\beta_{i}$ lies in $(0,1)$.

Moreover, suppose that $\left\|u_{n}-u_{n-1}\right\|=o\left(\epsilon_{n} \delta_{n} \beta_{n, k_{0}}\right)$ and (HO), (H7) and (H8) hold. Then, $\lim _{n \rightarrow \infty}\left\|S_{i} u_{n}-u_{n}\right\|=0$ for each $i=1, \ldots, N$ as $n \rightarrow \infty$.

Proof. First of all we note that if (H7) holds then also (H1)-(H6) are satisfied. So $\left\{x_{n}\right\}$ is asymptotically regular.

Let $k_{0}$ be as in the hypotheses. As in Proposition 3.11, for every index $i \in\{1, \ldots, N\}$ such that $\beta_{n, i} \rightarrow \beta_{i} \neq 0$ (which leads to $0<\liminf _{n \rightarrow \infty} \beta_{n, i} \leq \limsup _{n \rightarrow \infty} \beta_{n, i}<1$ ), one has $\| S_{i} u_{n}-$ $y_{n, i-1} \| \rightarrow 0$ as $n \rightarrow \infty$.

For all the other indices $i \leq k_{0}$, we can prove that $\left\|S_{i} u_{n}-y_{n, i-1}\right\| \rightarrow 0$ as $n \rightarrow \infty$ in a similar manner. By the relation (due to (3.31) and (3.40))

$$
\begin{aligned}
\left\|x_{n+1}-p\right\|^{2} & \leq\left(1-\epsilon_{n} \tau\right)\left\|y_{n}-p\right\|^{2}+\epsilon_{n} \tau \frac{1}{\tau^{2}}\left\|\gamma\left(\delta_{n} V x_{n}+\left(1-\delta_{n}\right) S x_{n}\right)-\mu F p\right\|^{2} \\
& \leq\left\|y_{n}-p\right\|^{2}+\epsilon_{n} \frac{1}{\tau}\left\|\gamma\left(\delta_{n} V x_{n}+\left(1-\delta_{n}\right) S x_{n}\right)-\mu F p\right\|^{2}
\end{aligned}
$$




$$
\begin{aligned}
\leq & \left\|x_{n}-p\right\|^{2}-\left(1-\beta_{n}\right) \beta_{n, i} \prod_{k=i}^{N}\left(1-\beta_{n, k}\right)\left\|S_{i} u_{n}-y_{n, i-1}\right\|^{2} \\
& +2 \lambda_{n} \alpha_{n}\|p\|\left\|\tilde{y}_{n, N}-p\right\|+\epsilon_{n} \frac{1}{\tau}\left\|\gamma\left(\delta_{n} V x_{n}+\left(1-\delta_{n}\right) S x_{n}\right)-\mu F p\right\|^{2} .
\end{aligned}
$$

we immediately obtain that

$$
\begin{aligned}
& (1-d) \prod_{k=i}^{N}\left(1-\beta_{n, k}\right)\left\|S_{i} u_{n}-y_{n, i-1}\right\|^{2} \\
& \leq\left(1-\beta_{n}\right) \prod_{k=i}^{N}\left(1-\beta_{n, k}\right)\left\|S_{i} u_{n}-y_{n, i-1}\right\|^{2} \\
& \leq \frac{\left\|x_{n}-p\right\|^{2}-\left\|x_{n+1}-p\right\|^{2}}{\beta_{n, i}}+2 \lambda_{n} \frac{\alpha_{n}}{\beta_{n, i}}\|p\|\left\|\tilde{y}_{n, N}-p\right\| \\
& \quad+\frac{\epsilon_{n}}{\beta_{n, i}} \frac{1}{\tau}\left\|\gamma\left(\delta_{n} V x_{n}+\left(1-\delta_{n}\right) S x_{n}\right)-\mu F p\right\|^{2} \\
& \leq \frac{\left\|x_{n}-x_{n+1}\right\|}{\beta_{n, i}}\left(\left\|x_{n}-p\right\|+\left\|x_{n+1}-p\right\|\right)+2 \lambda_{n} \frac{\alpha_{n}}{\beta_{n, i}}\|p\|\left\|\tilde{y}_{n, N}-p\right\| \\
& \quad+\frac{\epsilon_{n}}{\beta_{n, i}} \frac{1}{\tau}\left\|\gamma\left(\delta_{n} V x_{n}+\left(1-\delta_{n}\right) S x_{n}\right)-\mu F p\right\|^{2} .
\end{aligned}
$$

By Proposition 3.9 or by hypothesis (ii) on the sequences, we have

$$
\frac{\left\|x_{n}-x_{n+1}\right\|}{\beta_{n, i}}=\frac{\left\|x_{n}-x_{n+1}\right\|}{\beta_{n, k_{0}}} \cdot \frac{\beta_{n, k_{0}}}{\beta_{n, i}} \rightarrow 0 .
$$

So, the conclusion follows.

Remark 3.3. Let us consider $N=3$ and the following sequences:

(a) $\beta_{n}=\frac{1}{2}+\frac{2}{n^{2}}, \quad \gamma_{n}=\sigma_{n}=\frac{1}{4}-\frac{1}{n^{2}}, \quad \forall n>2$;

(b) $\lambda_{n}=\frac{1}{2\|A\|^{2}}-\frac{1}{2 n^{2}}, \quad \forall n>\|A\|$;

(c) $\alpha_{n}=\frac{1}{n^{2}}, \quad \epsilon_{n}=\frac{1}{n^{3 / 4}}, \quad \delta_{n}=\frac{1}{n^{1 / 4}}, \quad r_{n}=2-\frac{1}{n^{2}}, \quad \forall n>1$;

(d) $\beta_{n, 1}=\frac{1}{n^{1 / 4}}, \quad \beta_{n, 2}=\frac{1}{2}-\frac{1}{n^{2}}, \quad \beta_{n, 3}=\frac{1}{n^{1 / 3}}, \quad \forall n>1$.

It is easy to see that all hypotheses (i) - (iii), (H0), (H7) and (H8) of Proposition 3.12 are satisfied.

Remark 3.4. Under the hypotheses of Proposition 3.12, analogously to Proposition 3.11, one can see that

$$
\lim _{n \rightarrow \infty}\left\|S_{i} u_{n}-y_{n, i-1}\right\|=0, \quad \forall i \in\{2, \ldots, N\}
$$

Corollary 3.1. Let us suppose that the hypotheses of either Proposition 3.11 or Proposition 3.12 are satisfied. Then $\omega_{w}\left(x_{n}\right)=\omega_{w}\left(u_{n}\right)=\omega_{w}\left(y_{n, 1}\right), \omega_{s}\left(x_{n}\right)=\omega_{s}\left(u_{n}\right)=\omega_{s}\left(y_{n, 1}\right)$ and $\omega_{w}\left(x_{n}\right) \subset \Omega$. 
Proof. By Remark 3.1, we have $\omega_{w}\left(x_{n}\right)=\omega_{w}\left(u_{n}\right)$ and $\omega_{s}\left(x_{n}\right)=\omega_{s}\left(u_{n}\right)$. Note that by Remark 3.4 ,

$$
\lim _{n \rightarrow \infty}\left\|S_{N} u_{n}-y_{n, N-1}\right\|=0 .
$$

In the meantime, it is known that

$$
\lim _{n \rightarrow \infty}\left\|S_{N} u_{n}-u_{n}\right\|=\lim _{n \rightarrow \infty}\left\|u_{n}-x_{n}\right\|=\lim _{n \rightarrow \infty}\left\|x_{n}-y_{n}\right\|=0
$$

Hence we have

$$
\lim _{n \rightarrow \infty}\left\|S_{N} u_{n}-y_{n}\right\|=0 .
$$

Furthermore, it follows from (3.1) that

$$
\lim _{n \rightarrow \infty}\left\|y_{n, N}-y_{n, N-1}\right\|=\lim _{n \rightarrow \infty} \beta_{n, N}\left\|S_{N} u_{n}-y_{n, N-1}\right\|=0,
$$

which together with $\lim _{n \rightarrow \infty}\left\|S_{N} u_{n}-y_{n, N-1}\right\|=0$, yields

$$
\lim _{n \rightarrow \infty}\left\|S_{N} u_{n}-y_{n, N}\right\|=0 .
$$

Combining (3.42) and (3.43), we conclude that

$$
\lim _{n \rightarrow \infty}\left\|y_{n}-y_{n, N}\right\|=0
$$

which together with $\lim _{n \rightarrow \infty}\left\|x_{n}-y_{n}\right\|=0$, leads to

$$
\lim _{n \rightarrow \infty}\left\|x_{n}-y_{n, N}\right\|=0
$$

Combining (3.39) and (3.44), we get

$$
\lim _{n \rightarrow \infty}\left\|t_{n}-x_{n}\right\|=0
$$

Note that

$$
\begin{aligned}
y_{n}-x_{n} & =\gamma_{n}\left(z_{n}-x_{n}\right)+\sigma_{n}\left(T z_{n}-x_{n}\right) \\
& =\gamma_{n}\left(z_{n}-t_{n}\right)+\gamma_{n}\left(t_{n}-x_{n}\right)+\sigma_{n}\left(T z_{n}-T t_{n}\right)+\sigma_{n}\left(T t_{n}-x_{n}\right) .
\end{aligned}
$$

Passing to the norm and using Lemma 2.4 we get

$$
\begin{aligned}
\sigma_{n}\left\|T t_{n}-x_{n}\right\| & =\left\|y_{n}-x_{n}-\gamma_{n}\left(z_{n}-t_{n}\right)-\sigma_{n}\left(T z_{n}-T t_{n}\right)-\gamma_{n}\left(t_{n}-x_{n}\right)\right\| \\
& \leq\left\|y_{n}-x_{n}\right\|+\left\|\gamma_{n}\left(z_{n}-t_{n}\right)+\sigma_{n}\left(T z_{n}-T t_{n}\right)\right\|+\gamma_{n}\left\|t_{n}-x_{n}\right\| \\
& \leq\left\|y_{n}-x_{n}\right\|+\left(\gamma_{n}+\sigma_{n}\right)\left\|z_{n}-t_{n}\right\|+\gamma_{n}\left\|t_{n}-x_{n}\right\| \\
& \leq\left\|y_{n}-x_{n}\right\|+\left\|z_{n}-t_{n}\right\|+\left\|t_{n}-x_{n}\right\| .
\end{aligned}
$$


So, from (3.32), (3.38), (3.45) and $\liminf _{n \rightarrow \infty} \sigma_{n}>0$ it follows that

$$
\lim _{n \rightarrow \infty}\left\|T t_{n}-x_{n}\right\|=0
$$

which together with (3.45), yields

$$
\lim _{n \rightarrow \infty}\left\|T t_{n}-t_{n}\right\|=0
$$

Now we observe that

$$
\left\|x_{n}-y_{n, 1}\right\| \leq\left\|x_{n}-u_{n}\right\|+\left\|y_{n, 1}-u_{n}\right\|=\left\|x_{n}-u_{n}\right\|+\beta_{n, 1}\left\|S_{1} u_{n}-u_{n}\right\| .
$$

By Propositions 3.8 and 3.11, $\left\|x_{n}-u_{n}\right\| \rightarrow 0$ and $\left\|S_{1} u_{n}-u_{n}\right\| \rightarrow 0$ as $n \rightarrow \infty$, and hence

$$
\lim _{n \rightarrow \infty}\left\|x_{n}-y_{n, 1}\right\|=0 .
$$

So we get $\omega_{w}\left(x_{n}\right)=\omega_{w}\left(y_{n, 1}\right)$ and $\omega_{s}\left(x_{n}\right)=\omega_{s}\left(y_{n, 1}\right)$.

Let $p \in \omega_{w}\left(x_{n}\right)$. Then there exists a subsequence $\left\{x_{n_{i}}\right\}$ of $\left\{x_{n}\right\}$ such that $x_{n_{i}} \rightarrow p$. Since $p \in \omega_{w}\left(u_{n}\right)$, by Proposition 3.11 and Lemma 2.5 (demiclosedness principle), we have $p \in$ $\operatorname{Fix}\left(S_{i}\right)$ for each $i=1, \ldots, N$, i.e., $p \in \cap_{i=1}^{N} \operatorname{Fix}\left(S_{i}\right)$. Taking into account $p \in \omega_{w}\left(t_{n}\right)$ and $\| t_{n}-$ $G t_{n} \| \rightarrow 0$ (due to (3.38) and (3.45)), by Lemma 2.5 (demiclosedness principle) we know that $p \in \operatorname{Fix}(G)=: \operatorname{GSVI}(G)$. Also, since $p \in \omega_{w}\left(t_{n}\right)$ and $\left\|T t_{n}-t_{n}\right\| \rightarrow 0$ (due to (3.45) and (3.46)), by Lemma 2.3 (Demiclosedness principle) we get $p \in \operatorname{Fix}(T)$. Moreover, by Lemma 2.11 and Proposition 3.8 we know that $p \in \operatorname{GMEP}(\boldsymbol{\Theta}, h)$. Next we prove that $p \in \Gamma$. As a matter of fact, from (3.32) and (3.44) we know that $y_{n_{i}, N} \rightarrow p$ and $\tilde{y}_{n_{i}, N} \rightarrow p$. Let

$$
\widetilde{T} v= \begin{cases}\nabla f(\nu)+N_{C} v, & v \in C, \\ \varnothing, & v \notin C,\end{cases}
$$

where $N_{C} v=\{u \in H:\langle\nu-p, u\rangle \geq 0, \forall p \in C\}$. Then, $\widetilde{T}$ is maximal monotone and $0 \in \widetilde{T} v$ if and only if $v \in \operatorname{VI}(C, \nabla f)$; see [19] for more details. Let $(v, u) \in G(\widetilde{T})$. Since $u-\nabla f(v) \in N_{C} v$ and $\tilde{y}_{n, N} \in C$, we have

$$
\left\langle v-\tilde{y}_{n, N}, u-\nabla f(v)\right\rangle \geq 0 .
$$

On the other hand, from $\tilde{y}_{n, N}=P_{C}\left(I-\lambda_{n} \nabla f_{\alpha_{n}}\right) y_{n, N}$ and $v \in C$, we have

$$
\left\langle\nu-\tilde{y}_{n, N}, \tilde{y}_{n, N}-\left(y_{n, N}-\lambda_{n} \nabla f_{\alpha_{n}}\left(y_{n, N}\right)\right)\right\rangle \geq 0,
$$

and hence

$$
\left\langle v-\tilde{y}_{n, N}, \frac{\tilde{y}_{n, N}-y_{n, N}}{\lambda_{n}}+\nabla f_{\alpha_{n}}\left(y_{n, N}\right)\right\rangle \geq 0 .
$$

Therefore we have

$$
\left\langle v-\tilde{y}_{n_{i}, N}, u\right\rangle
$$




$$
\begin{aligned}
\geq & \left\langle v-\tilde{y}_{n_{i}, N}, \nabla f(v)\right\rangle \\
\geq & \left\langle v-\tilde{y}_{n_{i}, N}, \nabla f(v)\right\rangle-\left\langle v-\tilde{y}_{n_{i}, N}, \frac{\tilde{y}_{n_{i}, N}-y_{n_{i}, N}}{\lambda_{n_{i}}}+\nabla f_{\alpha_{n_{i}}}\left(y_{n_{i}, N}\right)\right\rangle \\
= & \left\langle v-\tilde{y}_{n_{i}, N}, \nabla f(v)\right\rangle-\left\langle v-\tilde{y}_{n_{i}, N}, \frac{\tilde{y}_{n_{i}, N}-y_{n_{i}, N}}{\lambda_{n_{i}}}+\nabla f\left(y_{n_{i}, N}\right)\right\rangle-\alpha_{n_{i}}\left\langle v-\tilde{y}_{n_{i}, N}, y_{n_{i}, N}\right\rangle \\
= & \left\langle v-\tilde{y}_{n_{i}, N}, \nabla f(v)-\nabla f\left(\tilde{y}_{n_{i}, N}\right)\right\rangle+\left\langle v-\tilde{y}_{n_{i}, N}, \nabla f\left(\tilde{y}_{n_{i}, N}\right)-\nabla f\left(y_{n_{i}, N}\right)\right\rangle \\
& -\left\langle v-\tilde{y}_{n_{i}, N}, \frac{\tilde{y}_{n_{i}, N}-y_{n_{i}, N}}{\lambda_{n_{i}}}\right\rangle-\alpha_{n_{i}}\left\langle v-\tilde{y}_{n_{i}, N}, y_{n_{i}, N}\right\rangle \\
\geq & \left\langle v-\tilde{y}_{n_{i}, N}, \nabla f\left(\tilde{y}_{n_{i}, N}\right)-\nabla f\left(y_{n_{i}, N}\right)\right\rangle-\left\langle v-\tilde{y}_{n_{i}, N}, \frac{\tilde{y}_{n_{i}, N}-y_{n_{i}, N}}{\lambda_{n_{i}}}\right\rangle-\alpha_{n_{i}}\left\langle v-\tilde{y}_{n_{i}, N}, y_{n_{i}, N}\right\rangle .
\end{aligned}
$$

From (3.32) and since $\nabla f$ is Lipschitz continuous, we obtain that $\lim _{n \rightarrow \infty} \| \nabla f\left(\tilde{y}_{n_{i}, N}\right)$ $-\nabla f\left(y_{n_{i}, N}\right) \|=0$. From $\tilde{y}_{n_{i}, N} \rightarrow p,\left\{\lambda_{n}\right\} \subset[a, b] \subset\left(0, \frac{1}{\|A\|^{2}}\right)$ and (3.32), we have

$$
\langle\nu-p, u\rangle \geq 0 \text {. }
$$

Since $\widetilde{T}$ is maximal monotone, we have $p \in \widetilde{T}^{-1} 0$ and hence $p \in \operatorname{VI}(C, \nabla f)$, which implies $p \in \Gamma$. Consequently, it is known that $p \in \operatorname{Fix}(T) \cap \bigcap_{i=1}^{N} \operatorname{Fix}\left(S_{i}\right) \cap \operatorname{GMEP}(\Theta, h) \cap$ $\operatorname{GSVI}(G) \cap \Gamma=: \Omega$.

Theorem 3.1. Let us suppose that $\Omega \neq \varnothing$. Let $\left\{\alpha_{n}\right\},\left\{\beta_{n, i}\right\}, i=1, \ldots, N$, be sequences in $(0,1)$ such that $0<\liminf _{n \rightarrow \infty} \beta_{n, i} \leq \limsup _{n \rightarrow \infty} \beta_{n, i}<1$ for each index $i$. Moreover, let us suppose that (H0)-(H6) hold. Then the sequences $\left\{x_{n}\right\},\left\{y_{n}\right\}$ and $\left\{u_{n}\right\}$ defined by scheme (3.1), all converge strongly to the unique solution $x^{*} \in \Omega$ of Problem 1.3 provided $\left\|x_{n}-x_{n-1}\right\|+\alpha_{n}=o\left(\epsilon_{n} \delta_{n}^{2}\right)$.

Proof. First of all, let us show that $\omega_{w}\left(x_{n}\right) \subset \Xi$. As a matter of fact, we note that $F: C \rightarrow H$ is $\eta$-strongly monotone and $\kappa$-Lipschitzian and $V: C \rightarrow H$ is a $\rho$-contraction with $\rho \in[0,1)$. Observe that

$$
\begin{aligned}
\mu \eta \geq \tau & \Leftrightarrow \mu \eta \geq 1-\sqrt{1-\mu\left(2 \eta-\mu \kappa^{2}\right)} \\
& \Leftrightarrow \sqrt{1-\mu\left(2 \eta-\mu \kappa^{2}\right)} \geq 1-\mu \eta \\
& \Leftrightarrow 1-2 \mu \eta+\mu^{2} \kappa^{2} \geq 1-2 \mu \eta+\mu^{2} \eta^{2} \\
& \Leftrightarrow \kappa^{2} \geq \eta^{2} \\
& \Leftrightarrow \kappa \geq \eta .
\end{aligned}
$$

Take a fixed $w \in \omega_{w}\left(x_{n}\right)$ arbitrarily. Then there exists a subsequence $\left\{x_{n_{i}}\right\}$ of $\left\{x_{n}\right\}$ such that $x_{n_{i}} \rightarrow w$. Hence, according to Corollary 3.1, we get $w \in \Omega$. Since $x_{n+1}=P_{C} v_{n}$ and $0<\gamma \leq \tau$, from (3.1) and (3.16), it follows that for all $p \in \Omega$

$$
\left\|x_{n+1}-p\right\|^{2}=\left\langle v_{n}-p, x_{n+1}-p\right\rangle+\left\langle P_{C} v_{n}-v_{n}, P_{C} v_{n}-p\right\rangle
$$




$$
\begin{aligned}
\leq & \left\langle v_{n}-p, x_{n+1}-p\right\rangle \\
= & \left\langle\left(I-\epsilon_{n} \mu F\right) y_{n}-\left(I-\epsilon_{n} \mu F\right) p, x_{n+1}-p\right\rangle \\
& +\epsilon_{n} \delta_{n} \gamma\left\langle V x_{n}-V p, x_{n+1}-p\right\rangle+\epsilon_{n}\left(1-\delta_{n}\right) \gamma\left\langle S x_{n}-S p, x_{n+1}-p\right\rangle \\
& +\epsilon_{n} \delta_{n}\left\langle(\gamma V-\mu F) p, x_{n+1}-p\right\rangle+\epsilon_{n}\left(1-\delta_{n}\right)\left\langle(\gamma S-\mu F) p, x_{n+1}-p\right\rangle \\
\leq & \left(1-\epsilon_{n} \tau\right)\left\|y_{n}-p\right\|\left\|x_{n+1}-p\right\| \\
& +\epsilon_{n} \delta_{n} \gamma \rho\left\|x_{n}-p\right\|\left\|x_{n+1}-p\right\|+\epsilon_{n}\left(1-\delta_{n}\right) \gamma\left\|x_{n}-p\right\|\left\|x_{n+1}-p\right\| \\
& +\epsilon_{n} \delta_{n}\left\langle(\gamma V-\mu F) p, x_{n+1}-p\right\rangle+\epsilon_{n}\left(1-\delta_{n}\right)\left\langle(\gamma S-\mu F) p, x_{n+1}-p\right\rangle \\
\leq & \left(1-\epsilon_{n} \tau\right)\left(\left\|x_{n}-p\right\|+\sqrt{2} \lambda_{n} \alpha_{n}\|p\|\right)\left\|x_{n+1}-p\right\| \\
& +\epsilon_{n} \delta_{n} \gamma \rho\left\|x_{n}-p\right\|\left\|x_{n+1}-p\right\|+\epsilon_{n}\left(1-\delta_{n}\right) \gamma\left\|x_{n}-p\right\|\left\|x_{n+1}-p\right\| \\
& +\epsilon_{n} \delta_{n}\left\langle(\gamma V-\mu F) p, x_{n+1}-p\right\rangle+\epsilon_{n}\left(1-\delta_{n}\right)\left\langle(\gamma S-\mu F) p, x_{n+1}-p\right\rangle \\
\leq & {\left[1-\epsilon_{n} \tau+\epsilon_{n} \delta_{n} \gamma \rho+\epsilon_{n}\left(1-\delta_{n}\right) \gamma\right]\left(\left\|x_{n}-p\right\|+\sqrt{2} \lambda_{n} \alpha \alpha_{n}\|p\|\right)\left\|x_{n+1}-p\right\| } \\
& +\epsilon_{n} \delta_{n}\left\langle(\gamma V-\mu F) p, x_{n+1}-p\right\rangle+\epsilon_{n}\left(1-\delta_{n}\right)\left\langle(\gamma S-\mu F) p, x_{n+1}-p\right\rangle \\
\leq & {\left[1-\epsilon_{n} \delta_{n} \gamma(1-\rho)\right]\left(\left\|x_{n}-p\right\|+\sqrt{2} \lambda_{n} \alpha_{n}\|p\|\right)\left\|x_{n+1}-p\right\| } \\
& +\epsilon_{n} \delta_{n}\left\langle(\gamma V-\mu F) p, x_{n+1}-p\right\rangle+\epsilon_{n}\left(1-\delta_{n}\right)\left\langle(\gamma S-\mu F) p, x_{n+1}-p\right\rangle \\
\leq & \left(\left\|x_{n}-p\right\|+\sqrt{2} \lambda_{n} \alpha x_{n}\|p\|\right)\left\|x_{n+1}-p\right\| \\
& +\epsilon_{n} \delta_{n}\left\langle(\gamma V-\mu F) p, x_{n+1}-p\right\rangle+\epsilon_{n}\left(1-\delta_{n}\right)\left\langle(\gamma S-\mu F) p, x_{n+1}-p\right\rangle .
\end{aligned}
$$

Thus, we have

$$
\begin{aligned}
& \left\langle(\mu F-\gamma S) p, x_{n+1}-p\right\rangle \\
& \leq \frac{\left(\left\|x_{n}-p\right\|+\sqrt{2} \lambda_{n} \alpha_{n}\|p\|-\left\|x_{n+1}-p\right\|\right)\left\|x_{n+1}-p\right\|}{\epsilon_{n}\left(1-\delta_{n}\right)}+\frac{\delta_{n}}{1-\delta_{n}}\left\langle(\gamma V-\mu F) p, x_{n+1}-p\right\rangle \\
& \leq \frac{\left(\left\|x_{n}-x_{n+1}\right\|+\sqrt{2} b \alpha_{n}\|p\|\right)\left\|x_{n+1}-p\right\|}{\epsilon_{n}\left(1-\delta_{n}\right)}+\frac{\delta_{n}}{1-\delta_{n}}\|(\gamma V-\mu F) p\|\left\|x_{n+1}-p\right\| .
\end{aligned}
$$

Since $\frac{\alpha_{n}}{\epsilon_{n}} \rightarrow 0, \frac{\left\|x_{n}-x_{n+1}\right\|}{\epsilon_{n}} \rightarrow 0, \delta_{n} \rightarrow 0$ and $x_{n_{i}} \rightarrow w$, we conclude from the boundedness of $\left\{x_{n}\right\}$ that for all $p \in \Omega$

$$
\begin{aligned}
& \langle(\mu F-\gamma S) p, w-p\rangle \\
& =\lim _{i \rightarrow \infty}\left\langle(\mu F-\gamma S) p, x_{n_{i}}-p\right\rangle \\
& \leq \limsup _{n \rightarrow \infty}\left\langle(\mu F-\gamma S) p, x_{n}-p\right\rangle \\
& =\limsup _{n \rightarrow \infty}\left(\left\langle(\mu F-\gamma S) p, x_{n+1}-p\right\rangle+\left\langle(\mu F-\gamma S) p, x_{n}-x_{n+1}\right\rangle\right) \\
& =\limsup _{n \rightarrow \infty}\left\langle(\mu F-\gamma S) p, x_{n+1}-p\right\rangle \\
& \leq \limsup _{n \rightarrow \infty}\left[\frac{\left(\left\|x_{n}-x_{n+1}\right\|+\sqrt{2} b \alpha_{n}\|p\|\right)\left\|x_{n+1}-p\right\|}{\epsilon_{n}\left(1-\delta_{n}\right)}+\frac{\delta_{n}}{1-\delta_{n}}\|(\gamma V-\mu F) p\|\left\|x_{n+1}-p\right\|\right]=0 .
\end{aligned}
$$


That is,

$$
\langle(\mu F-\gamma S) p, p-w\rangle \geq 0, \quad \forall p \in \Omega .
$$

Since $0<\gamma \leq \tau \leq \mu \eta$ and

$$
\langle(\mu F-\gamma S) x-(\mu F-\gamma S) y, x-y\rangle \geq(\mu \eta-\gamma)\|x-y\|^{2}, \quad \forall x, y \in C,
$$

we know that $\mu F-\gamma S$ is monotone and $(\mu \kappa+\gamma)$-Lipschitz continuous with constant $\mu \kappa+\gamma>0$. Thus, by Minty's lemma [43], we have

$$
\langle(\mu F-\gamma S) w, p-w\rangle \geq 0, \quad \forall p \in \Omega,
$$

which yields $w \in \Xi$. This shows that $\omega_{w}\left(x_{n}\right) \subset \Xi$. Furthermore, it is clear that

$$
\langle(\mu F-\gamma V) x-(\mu F-\gamma V) y, x-y\rangle \geq(\mu \eta-\gamma \rho)\|x-y\|^{2}, \quad \forall x, y \in C .
$$

So, it follows that $\mu F-\gamma V$ is $(\mu \eta-\gamma \rho)$-strongly monotone with constant $\mu \eta-\gamma \rho>0$. In the meantime, it is easy to see that $\mu F-\gamma V$ is $(\mu \kappa+\gamma \rho)$-Lipschitz continuous with constant $\mu \kappa+$ $\gamma \rho>0$. Thus, there exists a unique solution $x^{*}$ in $\Xi$ to the VIP

$$
\left\langle(\mu F-\gamma V) x^{*}, p-x^{*}\right\rangle \geq 0, \quad \forall p \in \Xi .
$$

Next, let us show that $\left\|x_{n}-x^{*}\right\| \rightarrow 0$ as $n \rightarrow \infty$. In fact, we now take a subsequence $\left\{x_{n_{j}}\right\}$ of $\left\{x_{n}\right\}$ satisfying

$$
\limsup _{n \rightarrow \infty}\left\langle(\gamma V-\mu F) x^{*}, x_{n}-x^{*}\right\rangle=\lim _{j \rightarrow \infty}\left\langle(\gamma V-\mu F) x^{*}, x_{n_{j}}-x^{*}\right\rangle .
$$

Without loss of generality, we may further assume that $x_{n_{j}}-\tilde{x}$; then $\tilde{x} \in \Xi$ according to the conclusion $\omega_{w}\left(x_{n}\right) \subset \Xi$ proved as above. So, it follows from (3.48) that

$$
\limsup _{n \rightarrow \infty}\left\langle(\gamma V-\mu F) x^{*}, x_{n}-x^{*}\right\rangle=\left\langle(\gamma V-\mu F) x^{*}, \tilde{x}-x^{*}\right\rangle \leq 0 .
$$

Putting $p=x^{*}$ in (3.47), we obtain from $0<\gamma \leq \tau$

$$
\begin{aligned}
&\left\|x_{n+1}-x^{*}\right\|^{2} \\
& \leq\left(1-\epsilon_{n} \tau\right)\left(\left\|x_{n}-x^{*}\right\|+\sqrt{2} \lambda_{n} \alpha_{n}\left\|x^{*}\right\|\right)\left\|x_{n+1}-x^{*}\right\| \\
&+\epsilon_{n} \delta_{n} \gamma \rho\left\|x_{n}-x^{*}\right\|\left\|x_{n+1}-x^{*}\right\|+\epsilon_{n}\left(1-\delta_{n}\right) \gamma\left\|x_{n}-x^{*}\right\|\left\|x_{n+1}-x^{*}\right\| \\
&+\epsilon_{n} \delta_{n}\left\langle(\gamma V-\mu F) x^{*}, x_{n+1}-x^{*}\right\rangle+\epsilon_{n}\left(1-\delta_{n}\right)\left\langle(\gamma S-\mu F) x^{*}, x_{n+1}-x^{*}\right\rangle \\
& \leq\left(1-\epsilon_{n} \tau\right)\left\|x_{n}-x^{*}\right\|\left\|x_{n+1}-x^{*}\right\|+\epsilon_{n} \delta_{n} \gamma \rho\left\|x_{n}-x^{*}\right\|\left\|x_{n+1}-x^{*}\right\| \\
&+\epsilon_{n}\left(1-\delta_{n}\right) \gamma\left\|x_{n}-x^{*}\right\|\left\|x_{n+1}-x^{*}\right\|+\epsilon_{n} \delta_{n}\left\langle(\gamma V-\mu F) x^{*}, x_{n+1}-x^{*}\right\rangle \\
&+\epsilon_{n}\left(1-\delta_{n}\right)\left\langle(\gamma S-\mu F) x^{*}, x_{n+1}-x^{*}\right\rangle+\sqrt{2} \lambda_{n} \alpha_{n}\left\|x^{*}\right\|\left\|x_{n+1}-x^{*}\right\|
\end{aligned}
$$




$$
\begin{aligned}
= & {\left[1-\epsilon_{n} \tau+\epsilon_{n} \delta_{n} \gamma \rho+\epsilon_{n}\left(1-\delta_{n}\right) \gamma\right]\left\|x_{n}-x^{*}\right\|\left\|x_{n+1}-x^{*}\right\|+\epsilon_{n} \delta_{n}\left\langle(\gamma V-\mu F) x^{*}, x_{n+1}-x^{*}\right\rangle } \\
& +\epsilon_{n}\left(1-\delta_{n}\right)\left\langle(\gamma S-\mu F) x^{*}, x_{n+1}-x^{*}\right\rangle+\sqrt{2} \lambda_{n} \alpha_{n}\left\|x^{*}\right\|\left\|x_{n+1}-x^{*}\right\| \\
\leq & {\left[1-\epsilon_{n} \delta_{n} \gamma(1-\rho)\right]\left\|x_{n}-x^{*}\right\|\left\|x_{n+1}-x^{*}\right\|+\epsilon_{n} \delta_{n}\left\langle(\gamma V-\mu F) x^{*}, x_{n+1}-x^{*}\right\rangle } \\
& +\epsilon_{n}\left(1-\delta_{n}\right)\left\langle(\gamma S-\mu F) x^{*}, x_{n+1}-x^{*}\right\rangle+\sqrt{2} \lambda_{n} \alpha_{n}\left\|x^{*}\right\|\left\|x_{n+1}-x^{*}\right\| \\
\leq & {\left[1-\epsilon_{n} \delta_{n} \gamma(1-\rho)\right] \frac{1}{2}\left(\left\|x_{n}-x^{*}\right\|^{2}+\left\|x_{n+1}-x^{*}\right\|^{2}\right)+\epsilon_{n} \delta_{n}\left\langle(\gamma V-\mu F) x^{*}, x_{n+1}-x^{*}\right\rangle } \\
& +\epsilon_{n}\left(1-\delta_{n}\right)\left\langle(\gamma S-\mu F) x^{*}, x_{n+1}-x^{*}\right\rangle+\sqrt{2} b \alpha_{n}\left\|x^{*}\right\|\left\|x_{n+1}-x^{*}\right\| .
\end{aligned}
$$

It turns out that

$$
\begin{aligned}
\left\|x_{n+1}-x^{*}\right\|^{2} \leq & \frac{1-\epsilon_{n} \delta_{n} \gamma(1-\rho)}{1+\epsilon_{n} \delta_{n} \gamma(1-\rho)}\left\|x_{n}-x^{*}\right\|^{2}+\frac{2}{1+\epsilon_{n} \delta_{n} \gamma(1-\rho)}\left[\epsilon_{n} \delta_{n}\left\langle(\gamma V-\mu F) x^{*}, x_{n+1}-x^{*}\right\rangle\right. \\
& \left.+\epsilon_{n}\left(1-\delta_{n}\right)\left\langle(\gamma S-\mu F) x^{*}, x_{n+1}-x^{*}\right\rangle\right]+\frac{1}{1+\epsilon_{n} \delta_{n} \gamma(1-\rho)} 2 \sqrt{2} b \alpha_{n}\left\|x^{*}\right\|\left\|x_{n+1}-x^{*}\right\| \\
\leq & {\left[1-\epsilon_{n} \delta_{n} \gamma(1-\rho)\right]\left\|x_{n}-x^{*}\right\|^{2}+\frac{2}{1+\epsilon_{n} \delta_{n} \gamma(1-\rho)}\left[\epsilon_{n} \delta_{n}\left\langle(\gamma V-\mu F) x^{*}, x_{n+1}-x^{*}\right\rangle\right.} \\
& \left.+\epsilon_{n}\left(1-\delta_{n}\right)\left\langle(\gamma S-\mu F) x^{*}, x_{n+1}-x^{*}\right\rangle\right]+2 \sqrt{2} b \alpha_{n}\left\|x^{*}\right\|\left\|x_{n+1}-x^{*}\right\| \\
= & {\left[1-\epsilon_{n} \delta_{n} \gamma(1-\rho)\right]\left\|x_{n}-x^{*}\right\|^{2}+\epsilon_{n} \delta_{n} \gamma(1-\rho) \cdot \frac{2}{\gamma(1-\rho)\left(1+\epsilon_{n} \delta_{n} \gamma(1-\rho)\right)} } \\
& \times\left\{\left\langle(\gamma V-\mu F) x^{*}, x_{n+1}-x^{*}\right\rangle+\frac{\left(1-\delta_{n}\right)\left\langle(\gamma S-\mu F) x^{*}, x_{n+1}-x^{*}\right\rangle}{\delta_{n}}\right\} \\
& +2 \sqrt{2} b \alpha_{n}\left\|x^{*}\right\|\left\|x_{n+1}-x^{*}\right\| .
\end{aligned}
$$

On the other hand, we notice the assumptions on the scheme (3.1) that $\lim _{n \rightarrow \infty}\left(\epsilon_{n}+\right.$ $\left.\alpha_{n}\right) / \delta_{n}^{2}=0$ and $\max _{1 \leq i \leq N}\left\|x-S_{i} x\right\| \geq \bar{k} \cdot d(x, \Omega), \forall x \in C$ for some $\bar{k}>0$. Then, for $x^{*} \in \Xi$, we obtain

$$
\begin{aligned}
\left\langle(\gamma S-\mu F) x^{*}, x_{n+1}-x^{*}\right\rangle & =\left\langle(\gamma S-\mu F) x^{*}, x_{n+1}-P_{\Omega} u_{n}\right\rangle+\left\langle(\gamma S-\mu F) x^{*}, P_{\Omega} u_{n}-x^{*}\right\rangle \\
& \leq\left\langle(\gamma S-\mu F) x^{*}, x_{n+1}-P_{\Omega} u_{n}\right\rangle \\
& \leq\left\|(\gamma S-\mu F) x^{*}\right\|\left\|x_{n+1}-P_{\Omega} u_{n}\right\| \\
& \leq\left\|(\gamma S-\mu F) x^{*}\right\|\left(\left\|x_{n+1}-u_{n}\right\|+\left\|u_{n}-P_{\Omega} u_{n}\right\|\right) \\
& =\left\|(\gamma S-\mu F) x^{*}\right\|\left(\left\|x_{n+1}-u_{n}\right\|+d\left(u_{n}, \Omega\right)\right) \\
& \leq\left\|(\gamma S-\mu F) x^{*}\right\|\left(\left\|x_{n+1}-x_{n}\right\|+\left\|x_{n}-u_{n}\right\|+\frac{1}{\bar{k}} \max _{1 \leq i \leq N}\left\|u_{n}-S_{i} u_{n}\right\|\right) \\
& \leq\left\|(\gamma S-\mu F) x^{*}\right\|\left(\left\|x_{n+1}-x_{n}\right\|+\left\|x_{n}-u_{n}\right\|+\frac{1}{\bar{k}} \sum_{i=1}^{N}\left\|u_{n}-S_{i} u_{n}\right\|\right) .(3.51
\end{aligned}
$$

From (H1), (H3) and $\left\|x_{n}-x_{n-1}\right\|=o\left(\epsilon_{n} \delta_{n}^{2}\right)$ we have

$$
\lim _{n \rightarrow \infty} \frac{\left\|x_{n}-x_{n-1}\right\|}{\epsilon_{n-1} \delta_{n-1}^{2}}=\lim _{n \rightarrow \infty} \frac{\left\|x_{n}-x_{n-1}\right\|}{\epsilon_{n} \delta_{n}^{2}} \frac{\epsilon_{n}}{\epsilon_{n-1}} \frac{\delta_{n}^{2}}{\delta_{n-1}^{2}}=0 .
$$


From (3.27), (H4) and $\left\|x_{n}-x_{n-1}\right\|=o\left(\epsilon_{n} \delta_{n}^{2}\right)$ we get

$$
\begin{aligned}
\frac{\left\|u_{n}-u_{n-1}\right\|}{\epsilon_{n} \delta_{n}} & \leq \frac{\left\|x_{n}-x_{n-1}\right\|}{\epsilon_{n} \delta_{n}}+L \frac{\left|r_{n}-r_{n-1}\right|}{r_{n} \epsilon_{n} \delta_{n}} \\
& \leq \frac{\left\|x_{n}-x_{n-1}\right\|}{\epsilon_{n} \delta_{n}}+L \frac{\left|r_{n}-r_{n-1}\right|}{\bar{r} \epsilon_{n} \delta_{n}} \rightarrow 0 \text { as } n \rightarrow \infty .
\end{aligned}
$$

That is, $\left\|u_{n}-u_{n-1}\right\|=o\left(\epsilon_{n} \delta_{n}\right)$. Also, from (3.31) we obtain

$$
\begin{aligned}
\left\|x_{n+1}-p\right\|^{2} \leq & \left\|x_{n}-p\right\|^{2}-\left(1-\epsilon_{n} \tau\right)\left\{( 1 - \beta _ { n } ) \left[\left\|x_{n}-u_{n}\right\|^{2}+\left(1-\lambda_{n}^{2}\left(\alpha_{n}+\|A\|^{2}\right)^{2}\right)\left\|y_{n, N}-\tilde{y}_{n, N}\right\|^{2}\right.\right. \\
& \left.\left.+v_{2}\left(2 \zeta_{2}-v_{2}\right)\left\|F_{2} t_{n}-F_{2} p\right\|^{2}+v_{1}\left(2 \zeta_{1}-v_{1}\right)\left\|F_{1} \tilde{t}_{n}-F_{1} \tilde{p}\right\|^{2}\right]+\frac{\beta_{n}}{1-\beta_{n}}\left\|y_{n}-x_{n}\right\|^{2}\right\} \\
& +2 \lambda_{n} \alpha_{n}\|p\|\left\|p-\tilde{y}_{n, N}\right\|+\epsilon_{n} \frac{1}{\tau}\left\|\gamma\left(\delta_{n} V x_{n}+\left(1-\delta_{n}\right) S x_{n}\right)-\mu F p\right\|^{2} \\
\leq & \left\|x_{n}-p\right\|^{2}-\left(1-\epsilon_{n} \tau\right)\left(1-\beta_{n}\right)\left\|x_{n}-u_{n}\right\|^{2}+2 b \alpha_{n}\|p\|\left\|p-\tilde{y}_{n, N}\right\| \\
& +\epsilon_{n} \frac{1}{\tau}\left\|\gamma\left(\delta_{n} V x_{n}+\left(1-\delta_{n}\right) S x_{n}\right)-\mu F p\right\|^{2},
\end{aligned}
$$

which leads to

$$
\begin{aligned}
& \left(1-\epsilon_{n} \tau\right)(1-d) \frac{\left\|x_{n}-u_{n}\right\|^{2}}{\delta_{n}^{2}} \\
& \leq\left(1-\epsilon_{n} \tau\right)\left(1-\beta_{n}\right) \frac{\left\|x_{n}-u_{n}\right\|^{2}}{\delta_{n}^{2}} \\
& \leq \frac{\left\|x_{n}-p\right\|^{2}-\left\|x_{n+1}-p\right\|^{2}}{\delta_{n}^{2}}+2 b \frac{\alpha_{n}}{\delta_{n}^{2}}\|p\|\left\|p-\tilde{y}_{n, N}\right\|+\frac{\epsilon_{n}}{\tau \delta_{n}^{2}}\left\|\gamma\left(\delta_{n} V x_{n}+\left(1-\delta_{n}\right) S x_{n}\right)-\mu F p\right\|^{2} \\
& \leq \frac{\left\|x_{n}-x_{n+1}\right\|}{\delta_{n}^{2}}\left(\left\|x_{n}-p\right\|+\left\|x_{n+1}-p\right\|\right)+2 b \frac{\alpha_{n}}{\delta_{n}^{2}}\|p\|\left\|p-\tilde{y}_{n, N}\right\| \\
& \quad+\frac{\epsilon_{n}}{\tau \delta_{n}^{2}}\left\|\gamma\left(\delta_{n} V x_{n}+\left(1-\delta_{n}\right) S x_{n}\right)-\mu F p\right\|^{2} .
\end{aligned}
$$

Since $\frac{\alpha_{n}}{\delta_{n}^{2}} \rightarrow 0, \frac{\epsilon_{n}}{\delta_{n}^{2}} \rightarrow 0$ and $\frac{\left\|x_{n+1}-x_{n}\right\|}{\delta_{n}^{2}} \rightarrow 0$ (due to (3.52)), we have

$$
\lim _{n \rightarrow \infty} \frac{\left\|x_{n}-u_{n}\right\|}{\delta_{n}}=0 .
$$

Repeating the same argument process as in (3.40), we obtain that (noting that $y_{n, 0}=u_{n}$ )

$$
\left\|y_{n}-p\right\|^{2} \leq\left\|x_{n}-p\right\|^{2}-\left(1-\beta_{n}\right) \beta_{n, N}\left(1-\beta_{n, N}\right)\left\|S_{N} u_{n}-y_{n, N-1}\right\|^{2}+2 \lambda_{n} \alpha_{n}\|p\|\left\|\tilde{y}_{n, N}-p\right\|,
$$

and for $i \in\{1, \ldots, N-1\}$,

$$
\left\|y_{n}-p\right\|^{2} \leq\left\|x_{n}-p\right\|^{2}-\left(1-\beta_{n}\right) \beta_{n, i} \prod_{k=i}^{N}\left(1-\beta_{n, k}\right)\left\|S_{i} u_{n}-y_{n, i-1}\right\|^{2}+2 \lambda_{n} \alpha_{n}\|p\|\left\|\tilde{y}_{n, N}-p\right\| .
$$

Substituting (3.54) and (3.55) in (3.31), respectively, we have

$$
\left\|x_{n+1}-p\right\|^{2} \leq\left(1-\epsilon_{n} \tau\right)\left\|y_{n}-p\right\|^{2}+\epsilon_{n} \frac{1}{\tau}\left\|\gamma\left(\delta_{n} V x_{n}+\left(1-\delta_{n}\right) S x_{n}\right)-\mu F p\right\|^{2}
$$




$$
\begin{aligned}
\leq & \left\|y_{n}-p\right\|^{2}+\epsilon_{n} \frac{1}{\tau}\left\|\gamma\left(\delta_{n} V x_{n}+\left(1-\delta_{n}\right) S x_{n}\right)-\mu F p\right\|^{2} \\
\leq & \left\|x_{n}-p\right\|^{2}-\left(1-\beta_{n}\right) \beta_{n, N}\left(1-\beta_{n, N}\right)\left\|S_{N} u_{n}-y_{n, N-1}\right\|^{2}+2 \lambda_{n} \alpha_{n}\|p\|\left\|\tilde{y}_{n, N}-p\right\| \\
& +\epsilon_{n} \frac{1}{\tau}\left\|\gamma\left(\delta_{n} V x_{n}+\left(1-\delta_{n}\right) S x_{n}\right)-\mu F p\right\|^{2},
\end{aligned}
$$

and for $i \in\{1, \ldots, N-1\}$,

$$
\begin{aligned}
\left\|x_{n+1}-p\right\|^{2} & \\
\leq & \left\|y_{n}-p\right\|^{2}+\epsilon_{n} \frac{1}{\tau}\left\|\gamma\left(\delta_{n} V x_{n}+\left(1-\delta_{n}\right) S x_{n}\right)-\mu F p\right\|^{2} \\
\leq & \left\|x_{n}-p\right\|^{2}-\left(1-\beta_{n}\right) \beta_{n, i} \prod_{k=i}^{N}\left(1-\beta_{n, k}\right)\left\|S_{i} u_{n}-y_{n, i-1}\right\|^{2}+2 \lambda_{n} \alpha_{n}\|p\|\left\|\tilde{y}_{n, N}-p\right\| \\
& +\epsilon_{n} \frac{1}{\tau}\left\|\gamma\left(\delta_{n} V x_{n}+\left(1-\delta_{n}\right) S x_{n}\right)-\mu F p\right\|^{2} .
\end{aligned}
$$

So, it follows that

$$
\begin{aligned}
& (1-d) \beta_{n, N}\left(1-\beta_{n, N}\right) \frac{\left\|S_{N} u_{n}-y_{n, N-1}\right\|^{2}}{\delta_{n}^{2}} \\
& \leq\left(1-\beta_{n}\right) \beta_{n, N}\left(1-\beta_{n, N}\right) \frac{\left\|S_{N} u_{n}-y_{n, N-1}\right\|^{2}}{\delta_{n}^{2}} \\
& \leq \frac{\left\|x_{n}-p\right\|^{2}-\left\|x_{n+1}-p\right\|^{2}}{\delta_{n}^{2}}+2 \lambda_{n} \frac{\alpha_{n}}{\delta_{n}^{2}}\|p\|\left\|\tilde{y}_{n, N}-p\right\|+\frac{\epsilon_{n}}{\tau \delta_{n}^{2}}\left\|\gamma\left(\delta_{n} V x_{n}+\left(1-\delta_{n}\right) S x_{n}\right)-\mu F p\right\|^{2} \\
& \leq \frac{\left\|x_{n}-x_{n+1}\right\|}{\delta_{n}^{2}}\left(\left\|x_{n}-p\right\|+\left\|x_{n+1}-p\right\|\right)+2 b \frac{\alpha_{n}}{\delta_{n}^{2}}\|p\|\left\|\tilde{y}_{n, N}-p\right\| \\
& \quad+\frac{\epsilon_{n}}{\delta_{n}^{2} \tau}\left\|\gamma\left(\delta_{n} V x_{n}+\left(1-\delta_{n}\right) S x_{n}\right)-\mu F p\right\|^{2},
\end{aligned}
$$

and for $i \in\{1, \ldots, N-1\}$,

$$
\begin{aligned}
& (1-d) \beta_{n, i} \prod_{k=i}^{N}\left(1-\beta_{n, k}\right) \frac{\left\|S_{i} u_{n}-y_{n, i-1}\right\|^{2}}{\delta_{n}^{2}} \\
& \leq\left(1-\beta_{n}\right) \beta_{n, i} \prod_{k=i}^{N}\left(1-\beta_{n, k}\right) \frac{\left\|S_{i} u_{n}-y_{n, i-1}\right\|^{2}}{\delta_{n}^{2}} \\
& \leq \frac{\left\|x_{n}-p\right\|^{2}-\left\|x_{n+1}-p\right\|^{2}}{\delta_{n}^{2}}+2 \lambda_{n} \frac{\alpha_{n}}{\delta_{n}^{2}}\|p\|\left\|\tilde{y}_{n, N}-p\right\|+\frac{\epsilon_{n}}{\delta_{n}^{2} \tau}\left\|\gamma\left(\delta_{n} V x_{n}+\left(1-\delta_{n}\right) S x_{n}\right)-\mu F p\right\|^{2} \\
& \leq \frac{\left\|x_{n}-x_{n+1}\right\|}{\delta_{n}^{2}}\left(\left\|x_{n}-p\right\|+\left\|x_{n+1}-p\right\|\right)+2 b \frac{\alpha_{n}}{\delta_{n}^{2}}\|p\|\left\|\tilde{y}_{n, N}-p\right\| \\
& \quad+\frac{\epsilon_{n}}{\tau \delta_{n}^{2}}\left\|\gamma\left(\delta_{n} V x_{n}+\left(1-\delta_{n}\right) S x_{n}\right)-\mu F p\right\|^{2} .
\end{aligned}
$$

Since $\frac{\alpha_{n}}{\delta_{n}^{2}} \rightarrow 0, \frac{\epsilon_{n}}{\delta_{n}^{2}} \rightarrow 0, \frac{\left\|x_{n+1}-x_{n}\right\|}{\delta_{n}^{2}} \rightarrow 0$ (due to (3.52)) and $0<\liminf _{n \rightarrow \infty} \beta_{n, i} \leq \limsup _{n \rightarrow \infty} \beta_{n, i}$ $<1$ for each $i \in\{1, \ldots, N\}$, we deduce that (noting that $y_{n, 0}=u_{n}$ )

$$
\lim _{n \rightarrow \infty} \frac{\left\|S_{i} u_{n}-y_{n, i-1}\right\|}{\delta_{n}}=0, \quad \forall i \in\{1, \ldots, N\} .
$$


Obviously for $i=1$, we have $\left\|S_{1} u_{n}-u_{n}\right\| / \delta_{n} \rightarrow 0$.

To conclude, we have that

$$
\frac{\left\|S_{2} u_{n}-u_{n}\right\|}{\delta_{n}} \leq \frac{\left\|S_{2} u_{n}-y_{n, 1}\right\|}{\delta_{n}}+\frac{\left\|y_{n, 1}-u_{n}\right\|}{\delta_{n}}=\frac{\left\|S_{2} u_{n}-y_{n, 1}\right\|}{\delta_{n}}+\frac{\beta_{n, 1}\left\|S_{1} u_{n}-u_{n}\right\|}{\delta_{n}}
$$

from which $\frac{\left\|S_{2} u_{n}-u_{n}\right\|}{\delta_{n}} \rightarrow 0$. Thus by induction $\frac{\left\|S_{i} u_{n}-u_{n}\right\|}{\delta_{n}} \rightarrow 0$ for all $i=2, \ldots, N$ since it is enough to observe that

$$
\begin{aligned}
\frac{\left\|S_{i} u_{n}-u_{n}\right\|}{\delta_{n}} & \leq \frac{\left\|S_{i} u_{n}-y_{n, i-1}\right\|}{\delta_{n}}+\frac{\left\|y_{n, i-1}-S_{i-1} u_{n}\right\|}{\delta_{n}}+\frac{\left\|S_{i-1} u_{n}-u_{n}\right\|}{\delta_{n}} \\
& \leq \frac{\left\|S_{i} u_{n}-y_{n, i-1}\right\|}{\delta_{n}}+\left(1-\beta_{n, i-1}\right) \frac{\left\|S_{i-1} u_{n}-y_{n, i-2}\right\|}{\delta_{n}}+\frac{\left\|S_{i-1} u_{n}-u_{n}\right\|}{\delta_{n}} .
\end{aligned}
$$

Furthermore, it is clear from (3.51) that

$$
\frac{\left\langle(\gamma S-\mu F) x^{*}, x_{n+1}-x^{*}\right\rangle}{\delta_{n}} \leq\left\|(\gamma S-\mu F) x^{*}\right\|\left(\frac{\left\|x_{n+1}-x_{n}\right\|}{\delta_{n}}+\frac{\left\|x_{n}-u_{n}\right\|}{\delta_{n}}+\frac{\sum_{i=1}^{N}\left\|u_{n}-S_{i} u_{n}\right\|}{\bar{k} \delta_{n}}\right) .
$$

Hence, from (3.52), (3.53) and $\frac{\left\|S_{i} u_{n}-u_{n}\right\|}{\delta_{n}} \rightarrow 0, \forall i\{1, \ldots, N\}$, that

$$
\limsup _{n \rightarrow \infty} \frac{\left\langle(\gamma S-\mu F) x^{*}, x_{n+1}-x^{*}\right\rangle}{\delta_{n}} \leq 0 .
$$

Thus, from (H0), (3.49), (3.57) and $\sum_{n=0}^{\infty} \alpha_{n}<\infty$ it can be readily seen that $\sum_{n=0}^{\infty} \epsilon_{n} \delta_{n} \gamma(1-\rho)=$ $\infty, \sum_{n=0}^{\infty} 2 \sqrt{2} b \alpha_{n}\left\|x^{*}\right\|\left\|x_{n+1}-x^{*}\right\|<\infty$ and

$$
\begin{gathered}
\limsup _{n \rightarrow \infty} \frac{2}{\gamma(1-\rho)\left(1+\epsilon_{n} \delta_{n} \gamma(1-\rho)\right)}\left\{\left\langle(\gamma V-\mu F) x^{*}, x_{n+1}-x^{*}\right\rangle\right. \\
\left.+\frac{\left(1-\delta_{n}\right)\left\langle(\gamma S-\mu F) x^{*}, x_{n+1}-x^{*}\right\rangle}{\delta_{n}}\right\} \leq 0 .
\end{gathered}
$$

Consequently, applying Lemma 2.8 to (3.50), we infer that the sequence $\left\{x_{n}\right\}$ converges strongly to $x^{*}$. This completes the proof.

In a similar way, we can conclude another theorem as follows.

Theorem 3.2. Let us suppose that $\Omega \neq \varnothing$. Let $\left\{\alpha_{n}\right\},\left\{\beta_{n, i}\right\}, i=1, \ldots, N$, be sequences in $(0,1)$ such that $\beta_{n, i} \rightarrow \beta_{i}$ for each index $i$ as $n \rightarrow \infty$. Suppose that there exists $k \in\{1, \ldots, N\}$ for which $\beta_{n, k} \rightarrow 0$ as $n \rightarrow \infty$. Let $k_{0} \in\{1, \ldots, N\}$ the largest index for which $\beta_{n, k_{0}} \rightarrow 0$. Moreover, let us suppose that (H0), (H7) and (H8) hold and

(i) $\frac{\epsilon_{n}}{\beta_{n, k_{0}}} \rightarrow 0$ as $n \rightarrow \infty$;

(ii) if $i \leq k_{0}$ and $\beta_{n, i} \rightarrow \beta_{i}$ then $\frac{\beta_{n, k_{0}}}{\beta_{n, i}} \rightarrow 0$ as $n \rightarrow \infty$;

(iii) if $\beta_{n, i} \rightarrow \beta_{i} \neq 0$ then $\beta_{i}$ lies in $(0,1)$.

Then the sequences $\left\{x_{n}\right\},\left\{y_{n}\right\}$ and $\left\{u_{n}\right\}$ defined by scheme (3.1), all converge strongly to the unique solution $x^{*} \in \Omega$ of Problem 1.3 provided $\left\|x_{n}-x_{n-1}\right\|+\alpha_{n}=o\left(\epsilon_{n} \delta_{n}^{2} \beta_{n, k_{0}}\right)$. 
Remark 3.5. Theorems 3.1 and 3.2 improve, extend, supplement and develop [8, Theorem 4.1], [24, Theorem 3.2], [31, Theorems 3.1 and 3.2] and [41, Theorems 3.12 and 3.13] in the following aspects.

(i) The multi-step iterative scheme in [24, Theorem 3.2] is extended to develop a hybrid extragradient iterative scheme (3.1) with regularization by virtue of Korpelevich's extragradient method, Mann's iteration method [25] and gradient projection method (GPM) with regularization. The iterative scheme (3.1) is based on Korpelevich's extragradient method, viscosity approximation method (see [22, 23]), Mann's iteration method, hybrid steepest-descent method (see $[2,25]$ ) and gradient-projection method (GPM) with regularization. In the meantime, the iterative scheme (3.1) is also the generalization, improvement and development of the iterative ones in [8, Theorem 4.1], [31, Theorems 3.1 and 3.2] and [41, Theorems 3.12 and 3.13].

(ii) The argument techniques in our Theorems 3.1 and ?? are very different from those techniques in [8, Theorem 4.1], [24, Theorem 3.2], [31, Theorems 3.1 and 3.2] and [41, Theorems 3.12 and 3.13] because we make use of the properties of strict pseudocontractions (see Lemmas 2.3 and 2.4), the ones of the resolvent operator associated with $\boldsymbol{\Theta}$ and $h$ (see Lemmas 2.9-2.11), the fixed point problem $x^{*}=G x^{*}$ ( $\Leftrightarrow$ GSVI (1.6)) (see Proposition 2.2), the equivalence of inclusion problem $0 \in \widetilde{T} v$ to the VIP $v \in \operatorname{VI}(C, \nabla f)$ for maximal monotone operator $\widetilde{T}$ (see (2.2)), the contractive coefficient estimates for the contractions $T^{\lambda}$ associating with nonexpansive mappings (see Lemma 2.7) and the new argument process in the proof of $\omega_{w}\left(x_{n}\right) \subset \Omega$ and $\omega_{w}\left(x_{n}\right) \subset \Xi$.

(iii) The problem of finding an element of $\operatorname{Fix}(T) \cap \cap_{i=1}^{N} \operatorname{Fix}\left(S_{i}\right) \cap \operatorname{GMEP}(\boldsymbol{\Theta}, h) \cap \Xi \cap \Gamma$ in our Theorems 3.1 and 3.2 is more general and more subtle than the one of finding a fixed point of a nonexpansive mapping $T$ in [8, Theorem 4.1], the one of finding an element of $\operatorname{Fix}(T) \cap$ $\cap_{i=1}^{N} \operatorname{Fix}\left(S_{i}\right) \cap \operatorname{GMEP}(\Theta, h)$ in [24, Theorems 3.2] (where $T$ is a nonexpansive mapping), the one of finding an element of $\operatorname{Fix}(T) \cap \cap_{i=1}^{N} \operatorname{Fix}\left(S_{i}\right) \cap \operatorname{GMEP}(\Theta, h)$ in $[41$, Theorems 3.12 and 3.13] (where $T$ is a nonexpansive mapping) and the one of finding an element of $\operatorname{Fix}(T) \cap$ $\cap_{i=1}^{N} \operatorname{Fix}\left(S_{i}\right) \cap \operatorname{GMEP}(\boldsymbol{\Theta}, h) \cap \Xi$ in [31, Theorems 3.1 and 3.2] (where $T$ is a strict pseudocontraction).

(iv) Our Theorems 3.1 and 3.2 generalizes [31, Theorems 3.1 and 3.2] and [41, Theorems 3.12 and 3.13] in from the nonexpansive mapping $T$ to the strict pseudocontraction $T$ and extend them to the setting of the THVIP (1.8) (defined over the set $\operatorname{Fix}(T) \cap \cap_{i=1}^{N} \operatorname{Fix}\left(S_{i}\right)$ ) with constraints of GMEP (1.3), SFP (1.5), and GSVI (1.6). In the meantime, our Theorems 3.1 and 3.2 extend the THVIP (defined over the set Fix $(T)$ ) in [8, Theorem 4.1] and the THVIP (defined over the set $\left.\operatorname{Fix}(T) \cap \cap_{i=1}^{N} \operatorname{Fix}\left(S_{i}\right)\right)$ in [24, Theorem 3.2] to Problem 1.3, i.e., the THVIP (defined over the set $\left.\operatorname{Fix}(T) \cap \cap_{i=1}^{N} \operatorname{Fix}\left(S_{i}\right)\right)$ with constraints of GMEP (1.3), SFP (1.5), and GSVI (1.6). 


\section{Applications}

For a given nonlinear mapping $\mathscr{A}: C \rightarrow H$, we consider the variational inequality problem (VIP) of finding $\bar{x} \in C$ such that

$$
\langle\mathscr{A} \bar{x}, y-\bar{x}\rangle \geq 0, \quad \forall y \in C .
$$

We will indicate with $\operatorname{VI}(C, \mathscr{A})$ the set of solutions of the VIP $(4.1)$.

Recall that if $u$ is a point in $C$, then the following relation holds:

$$
u \in \operatorname{VI}(C, \mathscr{A}) \Leftrightarrow u=P_{C}(I-\lambda \mathscr{A}) u, \quad \forall \lambda>0 .
$$

In the meantime, it is easy to see that the following relation holds:

$$
\text { GSVI (1.6) with } F_{2}=0 \Leftrightarrow \operatorname{VIP}(4.1) \text { with } \mathscr{A}=F_{1} \text {. }
$$

An operator $\mathscr{A}: C \rightarrow H$ is said to be an $\alpha$-inverse strongly monotone operator if there exists a constant $\alpha>0$ such that

$$
\langle\mathscr{A} x-\mathscr{A} y, x-y\rangle \geq \alpha\|\mathscr{A} x-\mathscr{A} y\|^{2}, \quad \forall x, y \in C .
$$

As an example, we recall that the $\alpha$-inverse strongly monotone operators are firmly nonexpansive mappings if $\alpha \geq 1$ and that every $\alpha$-inverse strongly monotone operator is also $\frac{1}{\alpha}$-Lipschitz continuous (see [34]).

Let us observe also that, if $\mathscr{A}$ is $\alpha$-inverse strongly monotone, the mappings $P_{C}(I-\lambda \mathscr{A})$ are nonexpansive for all $\lambda \in(0,2 \alpha]$ since they are compositions of nonexpansive mappings (see p.419 in [34]).

Let us consider $\tilde{S}_{1}, \ldots, \tilde{S}_{M}$ a finite number of nonexpansive self-mappings on $C$ and $A_{1}, \ldots$, $A_{N}$ be a finite number of $\alpha$-inverse strongly monotone operators. Let $T: C \rightarrow C$ be a $\xi$-strict pseudocontraction with fixed points. Let us consider the following mixed problem of finding $x^{*} \in \operatorname{Fix}(T) \cap \operatorname{GMEP}(\boldsymbol{\Theta}, h) \cap \operatorname{GSVI}(G) \cap \Gamma$ such that

$$
\left\{\begin{array}{l}
\left\langle\left(I-\tilde{S}_{1}\right) x^{*}, y-x^{*}\right\rangle \geq 0, \quad \forall y \in \operatorname{Fix}(T) \cap \operatorname{GMEP}(\boldsymbol{\Theta}, h) \cap \operatorname{GSVI}(G) \cap \Gamma, \\
\left\langle\left(I-\tilde{S}_{2}\right) x^{*}, y-x^{*}\right\rangle \geq 0, \quad \forall y \in \operatorname{Fix}(T) \cap \operatorname{GMEP}(\boldsymbol{\Theta}, h) \cap \operatorname{GSVI}(G) \cap \Gamma, \\
\cdots \\
\left\langle\left(I-\tilde{S}_{M}\right) x^{*}, y-x^{*}\right\rangle \geq 0, \quad \forall y \in \operatorname{Fix}(T) \cap \operatorname{GMEP}(\boldsymbol{\Theta}, h) \cap \operatorname{GSVI}(G) \cap \Gamma, \\
\left\langle A_{1} x^{*}, y-x^{*}\right\rangle \geq 0, \quad \forall y \in C, \\
\left\langle A_{2} x^{*}, y-x^{*}\right\rangle \geq 0, \quad \forall y \in C, \\
\cdots \\
\left\langle A_{N} x^{*}, y-x^{*}\right\rangle \geq 0, \quad \forall y \in C .
\end{array}\right.
$$

Let us call (SVI) the set of solutions of the $(M+N)$-system. This problem is equivalent to finding a common fixed point of $T,\left\{P_{\operatorname{Fix}(T) \cap \operatorname{GMEP}(\boldsymbol{\Theta}, h) \cap \operatorname{GSVI}(G) \cap \Gamma} \tilde{S}_{i}\right\}_{i=1}^{M},\left\{P_{C}\left(I-\lambda A_{i}\right)\right\}_{i=1}^{N}$. So we claim that the following holds. 
Theorem 4.1. Let us suppose that $\Omega=\operatorname{Fix}(T) \cap(\mathrm{SVI}) \cap \operatorname{GMEP}(\Theta, h) \cap \operatorname{GSVI}(G) \cap \Gamma \neq \varnothing$. Fix $\lambda>0$. Let $\left\{\alpha_{n}\right\},\left\{\beta_{n, i}\right\}, i=1, \ldots,(M+N)$, be sequences in $(0,1)$ such that $0<\liminf _{n \rightarrow \infty} \beta_{n, i} \leq$ $\limsup _{n \rightarrow \infty} \beta_{n, i}<1$ for all indices $i$. Moreover, let us suppose that $(\mathrm{H} 0)-(\mathrm{H} 6)$ hold. Then the sequences $\left\{x_{n}\right\},\left\{y_{n}\right\}$ and $\left\{u_{n}\right\}$ explicitly defined by scheme

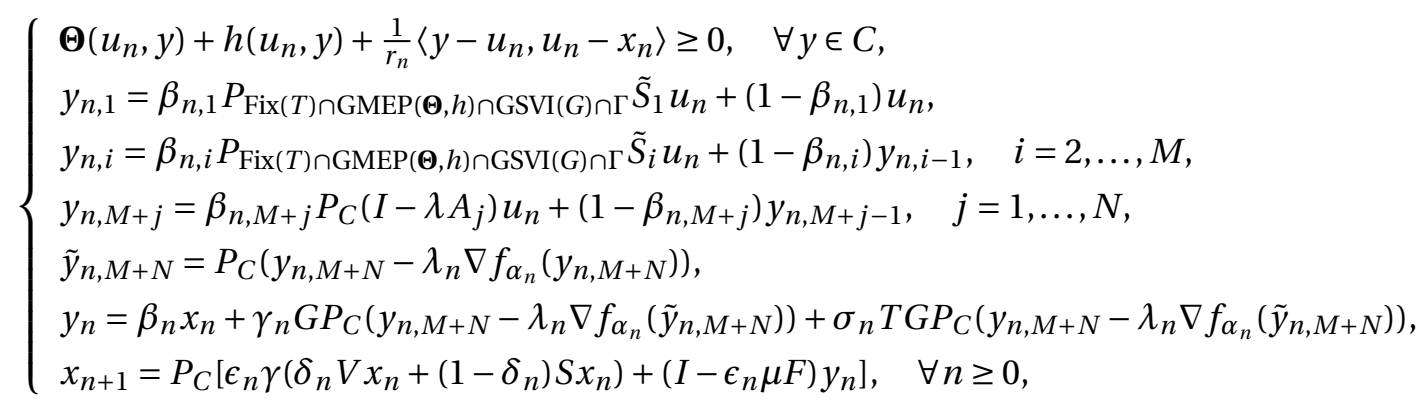

all converge strongly to the unique solution $x^{*} \in \Omega$ of Problem 1.3 provided $\left\|x_{n}-x_{n-1}\right\|+\alpha_{n}=$ $o\left(\epsilon_{n} \delta_{n}^{2}\right)$.

Theorem 4.2. Let us suppose that $\Omega \neq \varnothing$. Fix $\lambda>0$. Let $\left\{\alpha_{n}\right\},\left\{\beta_{n, i}\right\}, i=1, \ldots,(M+N)$, be sequences in $(0,1)$ and $\beta_{n, i} \rightarrow \beta_{i}$ for all $i$ as $n \rightarrow \infty$. Suppose that there exists $k \in\{1, \ldots, M+N\}$ such that $\beta_{n, k} \rightarrow 0$ as $n \rightarrow \infty$. Let $k_{0} \in\{1, \ldots, M+N\}$ be the largest index for which $\beta_{n, k_{0}} \rightarrow 0$. Moreover, let us suppose that (H0), (H7) and (H8) hold and

(i) $\frac{\epsilon_{n}}{\beta_{n, k_{0}}} \rightarrow 0$ as $n \rightarrow \infty$;

(ii) if $i \leq k_{0}$ and $\beta_{n, i} \rightarrow 0$ then $\frac{\beta_{n, k_{0}}}{\beta_{n, i}} \rightarrow 0$ as $n \rightarrow \infty$;

(iii) if $\beta_{n, i} \rightarrow \beta_{i} \neq 0$ then $\beta_{i}$ lies in $(0,1)$.

Then the sequences $\left\{x_{n}\right\},\left\{y_{n}\right\}$ and $\left\{u_{n}\right\}$ defined by scheme (3.1), all converge strongly to the unique solution $x^{*} \in \Omega$ of Problem 1.3 provided $\left\|x_{n}-x_{n-1}\right\|+\alpha_{n}=o\left(\epsilon_{n} \delta_{n}^{2} \beta_{n, k_{0}}\right)$.

Remark 4.1. If in system (4.3), $\nabla f=0, F_{1}=F_{2}=A_{1}=\cdots=A_{N}=0$ and $T$ is a nonexpansive mapping, we obtain a system of hierarchical fixed point problems introduced by Mainge and Moudafi [20, 21].

On the other hand, recall that a mapping $S: C \rightarrow C$ is called $\zeta$-strictly pseudocontractive if there exists a constant $\zeta \in[0,1)$ such that

$$
\|S x-S y\|^{2} \leq\|x-y\|^{2}+\zeta\|(I-S) x-(I-S) y\|^{2}, \quad \forall x, y \in C .
$$

If $\zeta=0$, then $S$ is nonexpansive. Put $\mathscr{A}=I-S$, where $S: C \rightarrow C$ is a $\zeta$-strictly pseudocontractive mapping. Then $\mathscr{A}$ is $\frac{1-\zeta}{2}$-inverse strongly monotone; see [37].

Utilizing Theorems 3.1 and 3.2, we also give two strong convergence theorems for finding a common element of the solution set $\operatorname{GMEP}(\Theta, h)$ of $\operatorname{GMEP}(1.3)$, the solution set $\Gamma$ of the 
SFP (1.5) and the common fixed point set $\cap_{i=1}^{N} \operatorname{Fix}\left(S_{i}\right) \cap \operatorname{Fix}(S)$ of finitely many nonexpansive mappings $S_{i}: C \rightarrow C, i=1, \ldots, N$, and a $\zeta$-strictly pseudocontractive mapping $S: C \rightarrow C$.

Theorem 4.3. Let $v_{1} \in(0,1-\zeta)$. Let us suppose that $\Omega=\cap_{i=1}^{N} \operatorname{Fix}\left(S_{i}\right) \cap \operatorname{Fix}(S) \cap \operatorname{GMEP}(\Theta, h) \cap \Gamma \neq$ $\varnothing$. Let $\left\{\alpha_{n}\right\},\left\{\beta_{n, i}\right\}, i=1, \ldots, N$, be sequences in $(0,1)$ such that $0<\liminf _{n \rightarrow \infty} \beta_{n, i} \leq \limsup _{n \rightarrow \infty}$ $\beta_{n, i}<1$ for all indices $i$. Moreover, let us suppose that there hold (HO)-(H6) with $\gamma_{n}=0, \forall n \geq 0$. Then the sequences $\left\{x_{n}\right\},\left\{y_{n}\right\}$ and $\left\{u_{n}\right\}$ generated explicitly by

$$
\left\{\begin{array}{l}
\boldsymbol{\Theta}\left(u_{n}, y\right)+h\left(u_{n}, y\right)+\frac{1}{r_{n}}\left\langle y-u_{n}, u_{n}-x_{n}\right\rangle \geq 0, \quad \forall y \in C, \\
y_{n, 1}=\beta_{n, 1} S_{1} u_{n}+\left(1-\beta_{n, 1}\right) u_{n}, \\
y_{n, i}=\beta_{n, i} S_{i} u_{n}+\left(1-\beta_{n, i}\right) y_{n, i-1}, \quad i=2, \ldots, N, \\
\tilde{y}_{n, N}=P_{C}\left(y_{n, N}-\lambda_{n} \nabla f_{\alpha_{n}}\left(y_{n, N}\right)\right), \\
t_{n}=P_{C}\left(y_{n, N}-\lambda_{n} \nabla f_{\alpha_{n}}\left(\tilde{y}_{n, N}\right),\right. \\
y_{n}=\beta_{n} x_{n}+\left(1-\beta_{n}\right)\left(\left(1-v_{1}\right) t_{n}+v_{1} S t_{n}\right), \\
x_{n+1}=P_{C}\left[\epsilon_{n} \gamma\left(\delta_{n} V x_{n}+\left(1-\delta_{n}\right) S x_{n}\right)+\left(I-\epsilon_{n} \mu F\right) y_{n}\right], \quad \forall n \geq 0,
\end{array}\right.
$$

all converge strongly to the unique solution $x^{*} \in \Omega$ of Problem 1.3 provided $\left\|x_{n}-x_{n-1}\right\|+\alpha_{n}=$ $o\left(\epsilon_{n} \delta_{n}^{2}\right)$.

Proof. In Theorem 3.1, put $F_{1}=\mathscr{A}=I-S$ and $F_{2}=0$. Then $\mathscr{A}$ is $\frac{1-\zeta}{2}$-inverse strongly monotone. Hence we deduce that $\operatorname{Fix}(S)=\operatorname{VI}(C, \mathscr{A})=\operatorname{GSVI}(G)$ and

$$
\begin{aligned}
G t_{n} & =P_{C}\left(I-v_{1} F_{1}\right) P_{C}\left(I-v_{2} F_{2}\right) t_{n} \\
& =P_{C}\left(I-v_{1} F_{1}\right) t_{n} \\
& =\left(1-v_{1}\right) t_{n}+v_{1} S t_{n} .
\end{aligned}
$$

Thus, in terms of Theorem 3.1, we obtain the desired result.

Theorem 4.4. Let $v_{1} \in(0,1-\zeta)$. Let us suppose that $\Omega=\cap_{i=1}^{N} \operatorname{Fix}\left(S_{i}\right) \cap \operatorname{Fix}(S) \cap \operatorname{GMEP}(\Theta, h) \cap \Gamma \neq$ $\varnothing$. Let $\left\{\alpha_{n}\right\},\left\{\beta_{n, i}\right\}, i=1, \ldots, N$, be sequences in $(0,1)$ such that $\beta_{n, i} \rightarrow \beta_{i}$ for all $i$ as $n \rightarrow \infty$. Suppose that there exists $k \in\{1, \ldots, N\}$ for which $\beta_{n, k} \rightarrow 0$ as $n \rightarrow \infty$. Let $k_{0} \in\{1, \ldots, N\}$ be the largest index for which $\beta_{n, k_{0}} \rightarrow 0$. Moreover, let us suppose that there hold (H0), (H7) and (H8) with $\gamma_{n}=0, \forall n \geq 0$ and

(i) $\frac{\epsilon_{n}}{\beta_{n, k_{0}}} \rightarrow 0$ as $n \rightarrow \infty$;

(ii) if $i \leq k_{0}$ and $\beta_{n, i} \rightarrow 0$ then $\frac{\beta_{n, k_{0}}}{\beta_{n, i}} \rightarrow 0$ as $n \rightarrow \infty$;

(iii) if $\beta_{n, i} \rightarrow \beta_{i} \neq 0$ then $\beta_{i}$ lies in $(0,1)$.

Then the sequences $\left\{x_{n}\right\},\left\{y_{n}\right\}$ and $\left\{u_{n}\right\}$ generated explicitly by (4.5), all converge strongly to the unique solution $x^{*} \in \Omega$ of Problem 1.3 provided $\left\|x_{n}-x_{n-1}\right\|+\alpha_{n}=o\left(\epsilon_{n} \delta_{n}^{2} \beta_{n, k_{0}}\right)$. 


\section{References}

[1] J. L. Lions, Quelques Méthodes de Résolution des Problèmes aux Limites Non Linéaires, Dunod, Paris, 1969.

[2] I. Yamada, The hybrid steepest-descent method for variational inequality problems over the intersection of the fixed-point sets of nonexpansive mappings, in: Inherently Parallel Algorithms in Feasibility and Optimization and Their Applications, edited by D. Butnariu, Y. Censor and S. Reich, North-Holland, Amsterdam, Holland, 473-504, 2001.

[3] H. K. Xu, Iterative methods for the split feasibility problem in infinite-dimensional Hilbert spaces, Inverse Problems, 26 (2010), 105018, 17 pp.

[4] Y. Censor and T. Elfving, A multiprojection algorithm using Bregman projections in a product space, Numer. Algorithms, 8 (1994), 221-239.

[5] L. C. Ceng, A. Petrusel and J. C. Yao, Iterative approaches to solving equilibrium problems and fixed point problems of infinitely many nonexpansive mappings, J. Optim. Theory Appl., 143 (2009), 37-58.

[6] C. Byrne, Iterative oblique projection onto convex sets and the split feasibility problem, Inverse Problems, 18 (2002), 441-453.

[7] C. Byrne, A unified treatment of some iterative algorithms in signal processing and image reconstruction, Inverse Problems, 20 (2004), 103-120.

[8] L. C. Ceng, Q. H. Ansari and J. C. Yao, Iterative methods for triple hierarchical variational inequalities in Hilbert spaces, J. Optim. Theory Appl., 151 (2011), 489-512.

[9] Y. Censor, A. Motova and A. Segal, Perturbed projections and subgradient projections for the multi-sets split feasibility problem, J. Math. Anal. Appl., 327 (2007), 1244-1256.

[10] N. Nadezhkina and W. Takahashi, Weak convergence theorem by an extragradient method for nonexpansive mappings and monotone mappings, J. Optim. Theory Appl., 128 (2006), 191-201.

[11] L. C. Ceng and J. C. Yao, Relaxed and hybrid viscosity methods for general system of variational inequalities with split feasibility problem constraint, Fixed Point Theory Appl., 2013, 2013:43, 50 pp.

[12] G. M. Korpelevich, The extragradient method for finding saddle points and other problems, Matecon., 12 (1976), 747-756.

[13] L. C. Ceng, M. M. Wong and J. C. Yao, A hybrid extragradient-like approximation method with regularization for solving split feasibility and fixed point problems, J. Nonlinear Convex Anal., 14 (2013), 1-20.

[14] R. W. Cottle, F. Giannessi and J. L. Lions, Variational Inequalities and Complementarity Problems: Theory and Applications, Wiley, New York, 1980.

[15] F. Facchinei and J.-S. Pang, Finite-Dimensional Variational Inequalities and Complementarity Problems, Vols. I and II, Springer, Berlin, 2003.

[16] A. Moudafi, Weak convergence theorems for nonexpansive mappings and equilibrium problems, J. Nonlinear Convex Anal., 9 (2008), 37-43.

[17] L. C. Ceng and J. C. Yao, A relaxed extragradient-like method for a generalized mixed equilibrium problem, a general system of generalized equilibria and a fixed point problem, Nonlinear Anal., 72 (2010), 1922-1937.

[18] L. C. Ceng and J. C. Yao, A hybrid iterative scheme for mixed equilibrium problems and fixed point problems, J. Comput. Appl. Math., 214 (2008), 186-201.

[19] R. T. Rockafellar, Monotone operators and the proximal point algorithms, SIAM J. Control Optim., 14 (1976), 877-898.

[20] A. Moudafi, P.-E. Mainge, Towards viscosity approximations of hierarchical fixed point problems, Fixed Point Theory Appl., 2006 (2006), Article ID 95453, 10 pp.

[21] A. Moudafi and P.-E. Mainge, Strong convergence of an iterative method for hierarchical fixed point problems, Pac. J. Optim., 3 (2007), 529-538.

[22] A. Moudafi, Viscosity approximation methods for fixed-points problems, J. Math. Anal. Appl., 241 (2000), 4655.

[23] H. K. Xu, Viscosity approximation methods for nonexpansive mappings, J. Math. Anal. Appl., 298 (2004), 279291. 
[24] A. Latif, L. C. Ceng and Q. H. Ansari, Multi-step hybrid viscosity method for systems of variational inequalities defined over sets of solutions of an equilibrium problem and fixed point problems, Fixed Point Theory Appl., 2012, 2012:78, $28 \mathrm{pp}$.

[25] H. K. Xu and T. H. Kim, Convergence of hybrid steepest-descent methods for variational inequalities, J. Optim. Theory. Appl., 119 (2003), 185-201.

[26] E. Blum and W. Oettli, From optimization and variational inequalities to equilibrium problems, Math. Student 63 (1994), 123-145.

[27] W. Oettli, A remark on vector-valued equilibria and generalized monotonicity, Acta Math. Vietnam. 22 (1997), 215-221.

[28] Y. Yao, Y.-C. Liou and G. Marino, Two-step iterative algorithms for hierarchical fixed point problems and variational inequality problems, J. Appl. Math. Comput., 31 (2009), 433-445.

[29] S. Atsushiba and W. Takahashi, Strong convergence theorems for a finite family of nonexpansive mappings and applications, in: B. N. Prasad Birth Centenary Commemoration Volume, Indian J. Math., 41 (1999), 435-453.

[30] Y. Censor, T. Bortfeld, B. Martin and A. Trofimov, A unified approach for inversion problems in intensitymodulated radiation therapy, Physics in Medicine and Biology, 51 (2006), 2353-2365.

[31] L. C. Ceng, Z. R. Kong and C. F. Wen, On general systems of variational inequalities, Comput. Math. Appl., 66 (2013), 1514-1532.

[32] C. Byrne, A unified treatment of some iterative algorithms in signal processing and image reconstruction, Inverse Problems, 20 (2004), 103-120.

[33] V. Colao, G. Marino and H. K. Xu, An iterative method for finding common solutions of equilibrium and fixed point problems, J. Math. Anal. Appl., 344 (2008), 340-352.

[34] W. Takahashi and M. Toyoda, Weak convergence theorems for nonexpansive mappings and monotone mappings, J. Optim. Theory Appl., 118 (2003), 417-428.

[35] H. Iiduka, Strong convergence for an iterative method for the triple-hierarchical constrained optimization problem, Nonlinear Anal., 71 (2009), 1292-1297.

[36] H. Iiduka, Iterative algorithm for solving triple-hierarchical constrained optimization problem, J. Optim. Theory Appl., 148 (2011), 580-592.

[37] G. Marino and $\mathrm{H}$. K. Xu, Weak and strong convergence theorems for strict pseudo-contractions in Hilbert spaces, J. Math. Math. Appl., 329 (2007), 336-346.

[38] Y. Yao, Y. C. Liou and S. M. Kang, Approach to common elements of variational inequality problems and fixed point problems via a relaxed extragradient method, Comput. Math. Appl., 59 (2010), 3472-3480.

[39] R. U. Verma, On a new system of nonlinear variational inequalities and associated iterative algorithms, Math. Sci. Res. Hot-Line, 3 (1999), 65-68.

[40] F. Cianciaruso, G. Marino, L. Muglia and Y. Yao, A hybrid projection algorithm for finding solutions of mixed equilibrium problem and variational inequality problem, Fixed Point Theory Appl., 2010 (2010), Article ID 383740, 19 pp.

[41] G. Marino, L. Muglia and Y. Yao, Viscosity methods for common solutions of equilibrium and variational inequality problems via multi-step iterative algorithms and common fixed points, Nonlinear Anal., 75 (2012), 1787-1798.

[42] L. C. Ceng, Q. H. Ansari, M. M. Wong and J. C. Yao, Mann type hybrid extragradient method for variational inequalities, variational inclusions and fixed point problems, Fixed Point Theory, 13 (2012), 403-422.

[43] K. Goebel and W. A. Kirk, Topics on Metric Fixed-Point Theory, Cambridge University Press, Cambridge, England, 1990.

[44] S. Takahashi and W. Takahashi, Strong convergence theorem for a generalized equilibrium problem and a nonexpansive mapping in a Hilbert space, Nonlinear Anal., 69 (2008), 1025-1033.

[45] L. C. Ceng, Q. H. Ansari and J. C. Yao, An extragradient method for solving split feasibility and fixed point problems, Comput. Math. Appl., 64 (2012), 633-642.

[46] L. C. Ceng, Q. H. Ansari and J. C. Yao, Relaxed extragradient methods for finding minimum-norm solutions of the split feasibility problem, Nonlinear Anal., 75 (2012), 2116-2125. 
[47] L. C. Ceng, Q. H. Ansari and J. C. Yao, Relaxed extragradient iterative methods for variational inequalities, Appl. Math. Comput., 218 (2011), 1112-1123.

[48] L. C. Ceng, Q. H. Ansari, N. C. Wong and J. C. Yao, An extragradient-like approximation method for variational inequalities and fixed point problems, Fixed Point Theory Appl., 2011, 2011:22, 18 pp.

[49] H. K. Xu, Iterative algorithms for nonlinear operators, J. Lond. Math. Soc., 66 (2002), 240-256.

[50] P. L. Combettes, Solving monotone inclusions via compositions of nonexpansive averaged operators, Optimization 53 (2004), 475-504.

Department of Mathematics, Shanghai Normal University, Shanghai 200234, China.

E-mail: zenglc@hotmail.com 